\title{
Facile sulfa-Michael reactions with sodium arylsulfinates in water: the promotion of water on the reaction
}

\author{
Guo-ping Lu, ${ }^{* \dagger}{ }^{\dagger}$ Chun Cai,${ }^{\dagger}$ Fei Chen, ${ }^{\ddagger \S}$ Ren-long Ye, ${ }^{\dagger}$ and Bao-jing Zhou ${ }^{* \dagger}$ \\ ${ }^{\dagger}$ Chemical Engineering College, Nanjing University of Science and Technology, Xiaolingwei 200, \\ Nanjing, 210094, China \\ ${ }^{*}$ Nanjing Institute of Environmental Sciences, Ministry of Environmental Protection, \\ Jiangwangmiao 8, Nanjing 210042, China \\ ${ }^{\S}$ Jiangsu Collaborative Innovation Center of Atmospheric Environment and Equipment \\ Technology (CICAEET), Ningliu 219, Nanjing, 210044, China \\ * Corresponding Author E-mail: glu@ @just.edu.cn; bzhou @njust.edu.cn
}

$\begin{array}{ll}\text { 1. Experimental } & \text { S2 }\end{array}$

2. Characterization Data S6

3. NMR Spectra of All Products $\quad$ S14 


\section{Experimental}

\subsection{General}

All chemical reagents were obtained from commercial suppliers and used without further purification. All known compounds were identified by appropriate technique such as MS, ${ }^{1} \mathrm{H}$ NMR, ${ }^{13} \mathrm{C}$ NMR and compared with previously reported data. All unknown compounds were characterized by ${ }^{1} \mathrm{H}$ NMR, ${ }^{13} \mathrm{C}$ NMR, MS and elemental analyses. Analytical thin-layer chromatography were performed on glass plates precoated with silica gel impregnated with a fluorescent indicator $(254 \mathrm{~nm})$, and the plates were visualized by exposure to ultraviolet light. Mass spectra were taken on a Finnigan TSQ Quantum-MS instrument in the electrospray ionization (ESI) mode. ${ }^{1} \mathrm{H}$ NMR and ${ }^{13} \mathrm{C}$ NMR spectra were recorded on an AVANCE 500 Bruker spectrometer operating at $500 \mathrm{MHz}$ and $125 \mathrm{MHz}$ in $\mathrm{CDCl}_{3}$, respectively, and chemical shifts were reported in ppm. Elemental analyses were performed on a Yanagimoto MT3CHN recorder.

QM geometry optimizations were performed using the Gaussian09 program $^{1}$ at the B3LYP/6-31G* level. The CSM calculations were carried out with the SMD method $^{2,3}$ using the $6-311++\mathrm{G}^{* *}$ basis set as implemented in the Gaussian09 program. In order to explore the possible reaction paths, we applied the AFIR method ${ }^{4-6}$ using the GRRM program ${ }^{7-9}$. In the AFIR method, a distant dependent energy term was employed to overcome the reaction barrier so that the association reaction can occur.

\subsection{Experimental Procedure}

General procedures for the sulfa-Michael reactions with sodium arylsulphinates: A mixture of activated alkene $\mathbf{2} 1.0 \mathrm{mmol}$ and sodium arylsulphinate $\mathbf{1} 1.2 \mathrm{mmol}$ was added in $1.2 \mathrm{M}$ hydrochloric acid solution $(1 \mathrm{~mL})$, which was stirred at room temperature for $8 \mathrm{~h}$. Upon completion, the precipitated was separated by filtration and washed with water to afford the pure products 3. In a few cases (liquid products), EtOAc $(5 \mathrm{~mL})$ was added after the reaction completed. The organic layer was collected and filtered through a bed of silica gel layered over Celite. The volatiles were removed in vacuo to afford the final product 3. (Z)-methyl 3-tosylacrylate 5 was synthesized under similar conditions.

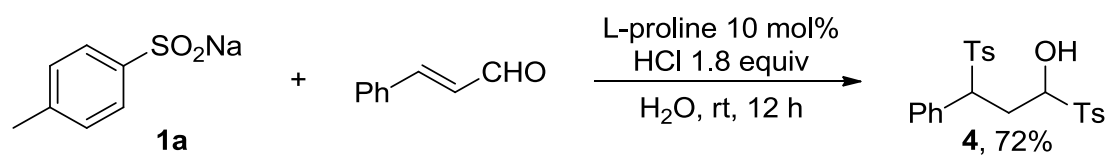

Synthesis of 4: A mixture of cinnamaldehyde $1.0 \mathrm{mmol}$, sodium phenyl sulphinate 1a $2.4 \mathrm{mmol}$ and L-proline $0.1 \mathrm{mmol}$ was added in $1.2 \mathrm{M}$ hydrochloric acid solution (1.5 $\mathrm{mL}$ ), which was stirred at room temperature for $12 \mathrm{~h}$. Upon completion, the precipitated was separated by filtration and washed with water to afford the pure products 4 . 


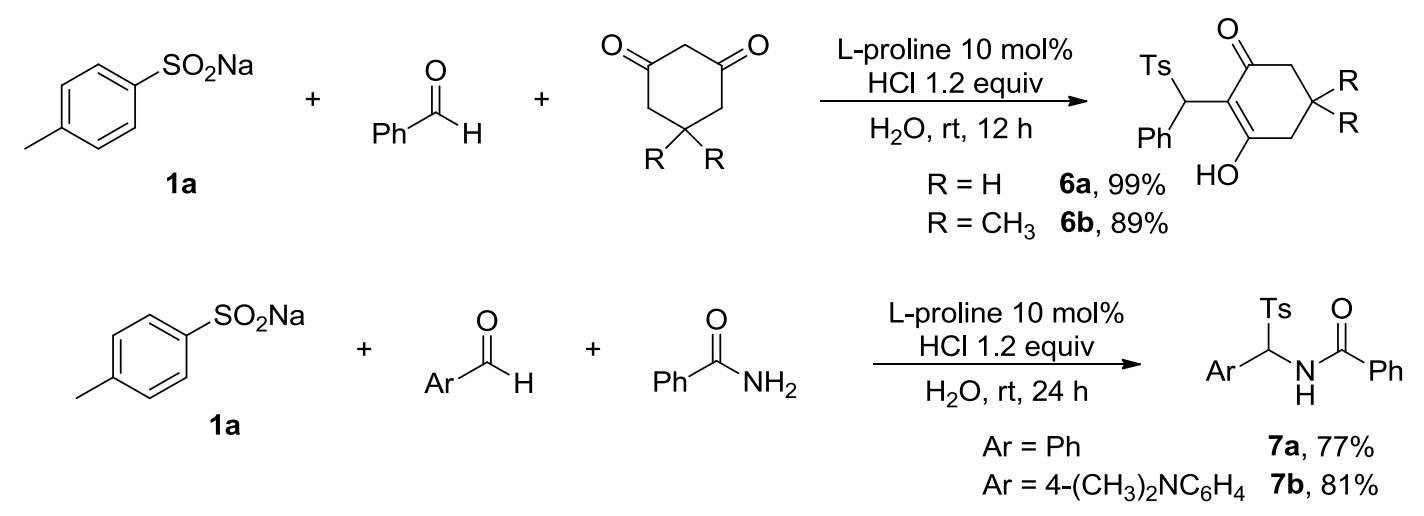

Synthesis of 6 and 7: A mixture of sodium phenyl sulphinate 1a $1.2 \mathrm{mmol}$, arylaldehyde $1.2 \mathrm{mmol}$, cyclohexadione (or benzamide) $1 \mathrm{mmol}$ and L-proline 0.1 mmol was added in 1.2 M hydrochloric acid solution $(1 \mathrm{~mL})$, which was stirred at room temperature for $12 \mathrm{~h}$ or $24 \mathrm{~h}$. Upon completion, the precipitated was separated by filtration and washed with water to afford the pure products 6 or 7 .

\subsection{The enantioselective sulfonation of $2 a$ with $1 \mathrm{a}$ in water}

Table S1. The optimization of reaction conditions $\mathrm{s}^{\mathrm{a}}$<smiles></smiles>

1a

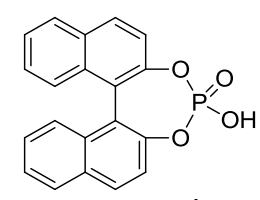

cat. 1<smiles>O=C(/C=C/c1ccccc1)c1ccccc1</smiles>

$2 a$

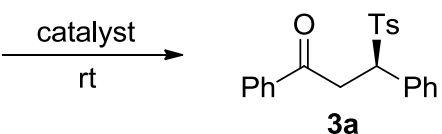<smiles>c1ccccc1</smiles>

L2

\begin{tabular}{|c|c|c|c|c|}
\hline entry & catalyst & solvent & yield $^{\mathrm{b}}$ & $e e^{\mathrm{c}}$ \\
\hline 1 & cat. 1 & $\mathrm{H}_{2} \mathrm{O}$ & 93 & 10 \\
\hline 2 & L-proline & $\mathrm{H}_{2} \mathrm{O}$ & NR & I \\
\hline $3^{\mathrm{d}}$ & $\mathrm{FeCl}_{3} / \mathbf{L 1} / \mathrm{TMSCl}$ & $\mathrm{H}_{2} \mathrm{O}$ & 94 & 0 \\
\hline $4^{\mathrm{d}}$ & $\mathrm{FeCl}_{3} / \mathbf{L} \mathbf{2} / \mathrm{TMSCl}$ & $\mathrm{H}_{2} \mathrm{O}$ & 91 & 0 \\
\hline 5 & cat. 1 & $\mathrm{EtOH}$ & trace & I \\
\hline 6 & cat. 1 & $\mathrm{MeOH}$ & trace & l \\
\hline 7 & cat. 1 & $\mathrm{MeCN}$ & trace & l \\
\hline 8 & cat. 1 & $\mathrm{DMF}$ & trace & l \\
\hline 9 & cat. 1 & $\mathrm{CH}_{2} \mathrm{Cl}_{2}$ & trace & I \\
\hline
\end{tabular}

${ }^{\mathrm{a}}$ 1a $1.2 \mathrm{mmol}$, 2a $1.0 \mathrm{mmol}$, catalyst $0.2 \mathrm{mmol}, \mathrm{rt}, 18 \mathrm{~h} .{ }^{\mathrm{b}}$ Isolated yields. ${ }^{\mathrm{c}}$ The enantiomeric ratio was determined by HPLC on a chiral phase. ${ }^{\mathrm{d}}$ The amounts of $\mathrm{FeCl}_{3}, \mathbf{L 1}$ and $\mathbf{L 2}$ were $20 \mathrm{~mol} \%$, and The amounts of TMSCl was 1.2 equiv. 


\subsection{Quantum chemical calculations}

Table S2 Four possible reaction routes depending on the protonation states of $\mathbf{1 a}$ and $\mathbf{2 a}$

entry route protonation states

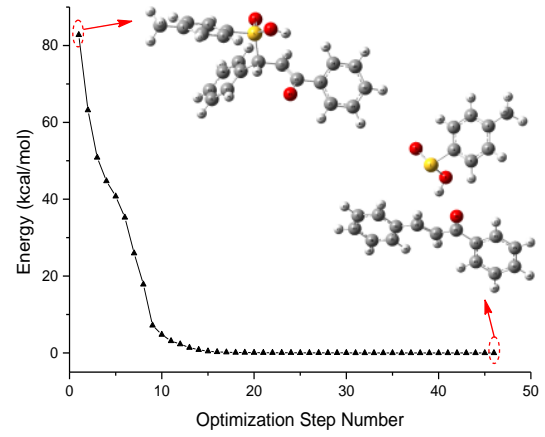

route $\mathbf{I}$

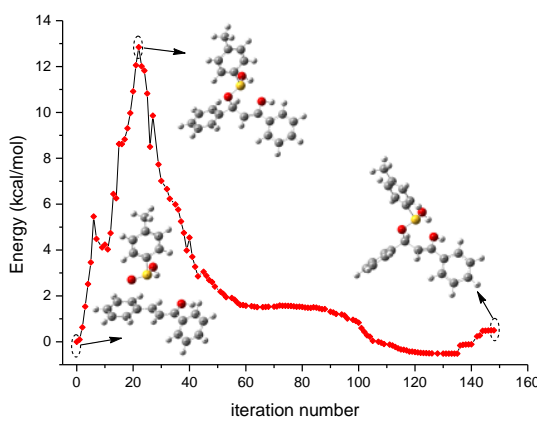

route III

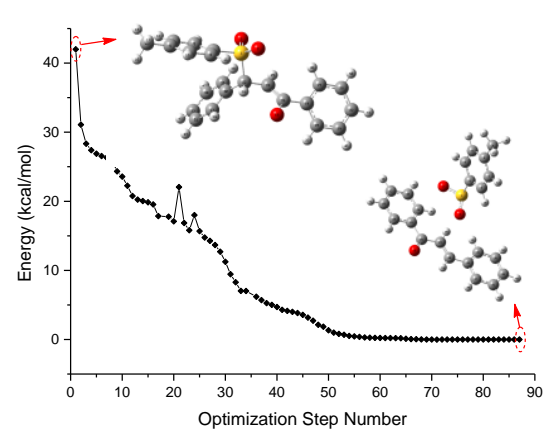

route II

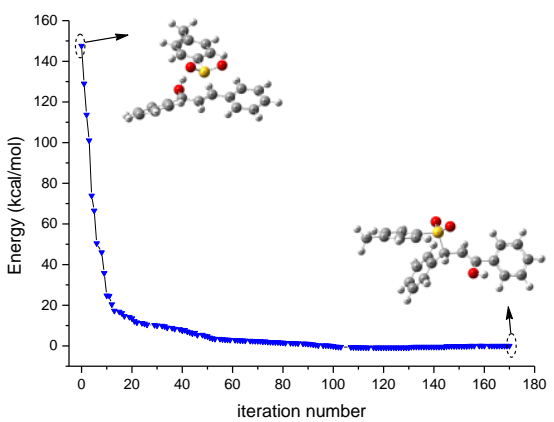

route IV

Figure S1 Potential energy profiles along AFIR paths for routes I-IV calculated using GRRM software and Gaussian09 program package. 
We considered the two possible protonation states for reactant 1a and 2a in the reaction. Their combination (Table S2) led to four possible reaction pathways. In pathway I and II, the AFIR method fails to associate the two reactants and no product is formed. Then we started from the product, which was subject to DFT geometry optimization. It is found the C-S bond is broken, and the reactants are produced since the potential energy of the product is much higher than that of the reactants by 40-80 $\mathrm{kcal} / \mathrm{mol}$ (Figure $\mathrm{S} 1$, routes I and II).

When the two protonated molecules reacted with each other (route III), it was the $\mathrm{C}-\mathrm{O}$ bond rather than the $\mathrm{C}-\mathrm{S}$ bond that was formed in the product as predicted by the AFIR method. Clearly, when 1a is protonated, its $\mathrm{S}$ atom becomes less electrophilic while the protonated olefin molecule becomes more reactive. The resulting product is not stable and the reaction barrier is about $13 \mathrm{kcal} / \mathrm{mol}$. In pathway $\mathbf{I V}, \mathbf{1 a}$ is in its ionic form, and 2a is protonated. As disclosed by DFT calculations, the product is much more stable than the reactant. No reaction barrier is found in the reaction pathway (IV) predicted by the AFIR method, which is consistent with the fact that the reaction can occur at room temperature. Thus, the protonation of chalcone (2a) is critical for the reaction to occur.

Table S3. Calculated solvation energies $(\mathrm{kcal} / \mathrm{mol})$ of the ionic reactant complex and product in various solvents using the SMD method

\begin{tabular}{ccccc}
\hline \multicolumn{5}{c}{$\Delta G_{\text {solv }}^{\text {tot }}$} \\
entry & solvent & ionic reactant complex & product & $\Delta \Delta G_{\text {solv }}^{\text {tot }}$ \\
& & -17.03 & -17.50 & -0.47 \\
\hline 1 & toluene & -15.60 & -15.97 & -0.37 \\
2 & hexane & -18.91 & -19.40 & -0.49 \\
3 & THF & -19.71 & -21.81 & -2.10 \\
4 & EtOH & -19.43 & -19.88 & -0.45 \\
5 & DMF & -11.36 & -12.00 & -0.64 \\
6 & 1,4-dioxane & -12.97 & -14.89 & -1.92 \\
7 & water & &
\end{tabular}

The relative solvation energies of the ionic reactant complex and the product in different solvents are now provided in Table S3. The product is much more stable than the reactant complex as reflected in Figure S1 route IV. Now, we show that their solvation energies are similar in various solvents, so the product is still much more stable than the reactant in different solvents. 


\section{Characterization Data}

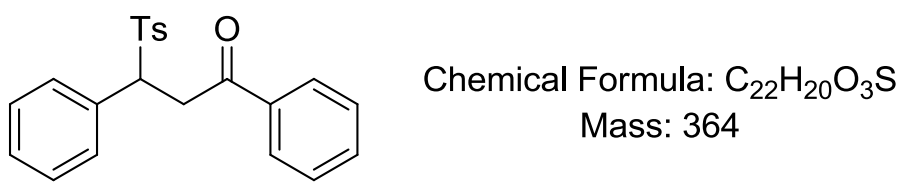

1,3-diphenyl-3-tosylpropan-1-one 3a, ${ }^{10}$ white solid, mp: $176-178{ }^{\circ} \mathrm{C}$ (lit. $179{ }^{\circ} \mathrm{C}$ ), yield $98 \%, 357$ mg. ${ }^{1} \mathrm{H}$ NMR $\left(\mathrm{CDCl}_{3}, 500 \mathrm{MHz}\right) \delta 2.31(\mathrm{~s}, 3 \mathrm{H}), 3.83-3.88(\mathrm{~m}, 1 \mathrm{H}), 4.01-4.06(\mathrm{~m}, 1 \mathrm{H}), 4.83-4.86$ $(\mathrm{dd}, J=8.0,3.5 \mathrm{~Hz}, 1 \mathrm{H}), 7.09-7.19(\mathrm{~m}, 7 \mathrm{H}), 7.34-7.40(\mathrm{~m}, 4 \mathrm{H}), 7.48-7.51(\mathrm{~m}, 1 \mathrm{H}), 7.85-7.86(\mathrm{~d}, J$ $=8.0 \mathrm{~Hz}, 2 \mathrm{H}) .{ }^{13} \mathrm{C} \mathrm{NMR}\left(\mathrm{CDCl}_{3}, 125 \mathrm{MHz}\right) \delta 21.7,37.2,66.6,128.3,128.6,128.9(3 \mathrm{C}), 129.2$, $129.5,129.9,132.8,133.8,134.2,136.3,144.8,195.1$. ESI-MS: $\mathrm{m} / \mathrm{z}=365[\mathrm{M}+1]^{+}$.

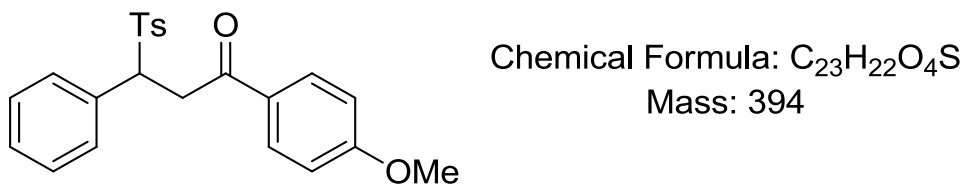

1-(4-methoxyphenyl)-3-phenyl-3-tosylpropan-1-one 3b, ${ }^{11}$ white solid, mp: 194-196 ${ }^{\circ} \mathrm{C}$ (lit. 198 $\left.{ }^{\circ} \mathrm{C}\right)$, yield 96\%, $378 \mathrm{mg} .{ }^{1} \mathrm{H}$ NMR $\left(\mathrm{CDCl}_{3}, 500 \mathrm{MHz}\right) \delta 2.38(\mathrm{~s}, 3 \mathrm{H}), 3.86(\mathrm{~s}, 3 \mathrm{H}), 3.87-3.91(\mathrm{~m}$, $1 \mathrm{H})$, 4.02-4.06 (m, 1H), 4.90-4.92 (m, 1H), 6.91-6.93 (d, $J=9.0 \mathrm{~Hz}, 2 \mathrm{H}), 7.16-7.27(\mathrm{~m}, 7 \mathrm{H})$, 7.41-7.43 (d, $J=8.0 \mathrm{~Hz}, 2 \mathrm{H}), 7.91-7.93(\mathrm{~d}, J=9.0 \mathrm{~Hz}, 2 \mathrm{H}) .{ }^{13} \mathrm{C}$ NMR $\left(\mathrm{CDCl}_{3}, 125 \mathrm{MHz}\right) \delta 21.7$, 36.7, 55.6, 66.7, 114.0, 128.5, 128.8, 129.1, 129.5, 129.9, 130.6, 132.9, 134.2, 144.8, 164.0, 193.5. ESI-MS: $\mathrm{m} / \mathrm{z}=395[\mathrm{M}+1]^{+}$.

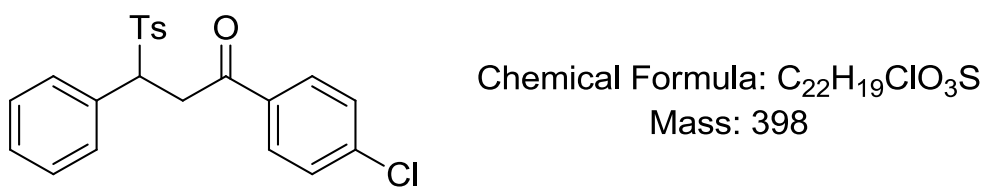

1-(4-chlorophenyl)-3-phenyl-3-tosylpropan-1-one 3c, ${ }^{11}$ white solid, mp: 188-190 ${ }^{\circ} \mathrm{C}$ (lit. 190-191 $\left.{ }^{\circ} \mathrm{C}\right)$, yield 99\%, $394 \mathrm{mg} .{ }^{1} \mathrm{H}$ NMR $\left(\mathrm{CDCl}_{3}, 500 \mathrm{MHz}\right) \delta 2.38(\mathrm{~s}, 3 \mathrm{H}), 3.84-3.90(\mathrm{~m}, 1 \mathrm{H}), 4.07-4.12$ (m, 1H), 4.88-4.90 (m, 1H), 7.16-7.27 (m, 7H), 7.40-7.44 (m, 4H), 7.87-7.88 (d, J= 8.5 Hz, 2H). ${ }^{13} \mathrm{C} \mathrm{NMR}\left(\mathrm{CDCl}_{3}, 125 \mathrm{MHz}\right) \delta 21.7,37.1,66.6,128.6,129.0,129.2,129.5,129.7,129.9,132.7$, 134.0, 134.6, 140.3, 144.9, 194.0. ESI-MS: $\mathrm{m} / \mathrm{z}=399[\mathrm{M}+1]^{+}$.<smiles>O=C(CC([18F])c1ccccn1)c1ccccc1</smiles>

\section{Chemical Formula: $\mathrm{C}_{21} \mathrm{H}_{19} \mathrm{NO}_{3} \mathrm{~S}$} Exact Mass: 365.1086

Elemental Analysis: C, 69.02; $\mathrm{H}, 5.24 ; \mathrm{N}$, 3.83; O, 13.13; S, 8.77

1-phenyl-3-(pyridin-2-yl)-3-tosylpropan-1-one 3d, white solid, mp: 185-187 ${ }^{\circ} \mathrm{C}$, yield 81\%, 296 mg. ${ }^{1} \mathrm{H}$ NMR $\left(\mathrm{CDCl}_{3} / \mathrm{DMSO}_{-} \mathrm{d}_{6}, 500 \mathrm{MHz}\right) \delta 2.37(\mathrm{~s}, 3 \mathrm{H}), 3.77-3.81(\mathrm{~d}, J=18 \mathrm{~Hz}, 1 \mathrm{H})$, $4.25-5.31(\mathrm{dd}, J=18.0,10.0 \mathrm{~Hz}, 1 \mathrm{H}), 5.27-5.29(\mathrm{~d}, J=10.0 \mathrm{~Hz}, 1 \mathrm{H}), 7.31-7.33(\mathrm{~d}, J=8.0 \mathrm{~Hz}, 2 \mathrm{H})$ 7.43-7.62 (m, 7H), 7.88-7.90 (m, 3H), 8.45-8.46 (d, $J=4.0 \mathrm{~Hz}, 1 \mathrm{H}) .{ }^{13} \mathrm{C}$ NMR $\left(\mathrm{CDCl}_{3} / \mathrm{DMSO}_{6} \mathrm{~d}_{6}\right.$, $125 \mathrm{MHz}) \delta 21.7,36.7,66.8,124.8,126.1,127.3,128.5,129.2,129.5,130.2,134.1,136.3,139.2$, 145.4, 147.8, 151.0, 195.7. ESI-MS: $\mathrm{m} / \mathrm{z}=366[\mathrm{M}+1]^{+}$. Anal. Calcd for $\mathrm{C}_{21} \mathrm{H}_{19} \mathrm{NO}_{3} \mathrm{~S}: \mathrm{C}, 69.02 \%$; H, 5.24\%; N, 3.83\%. Found: C, 68.78\%; H, 5.41\%; N, 3.99\%. 
<smiles>CC(=O)CC([18F])c1ccccc1</smiles>

\section{Chemical Formula: $\mathrm{C}_{17} \mathrm{H}_{18} \mathrm{O}_{3} \mathrm{~S}$ \\ Mass: 302}

4-phenyl-4-tosylbutan-2-one 3e, ${ }^{11}$ white solid, mp: 120-122 ${ }^{\circ} \mathrm{C}$ (lit. 123-125), yield 92\%, $278 \mathrm{mg}$. ${ }^{1} \mathrm{H}$ NMR $\left(\mathrm{CDCl}_{3}, 500 \mathrm{MHz}\right) \delta 2.14(\mathrm{~s}, 3 \mathrm{H}), 2.37(\mathrm{~s}, 3 \mathrm{H}), 3.22-3.28(\mathrm{~m}, 1 \mathrm{H}), 3.55-3.60(\mathrm{~m}, 1 \mathrm{H})$, 4.69-4.71 (m, 1H), 7.10-7.25 (m, 7H), 7.36-7.37 (d, $\left.J=8.0 \mathrm{~Hz}, 2 \mathrm{H}) .{ }^{13} \mathrm{C} \mathrm{NMR}_{(\mathrm{CDCl}}, 125 \mathrm{MHz}\right)$ $\delta 21.7,30.6,41.7,66.3,128.6,128.9,129.1,129.5,129.8,132.7,134.0,144.9,203.4$. ESI-MS: $\mathrm{m} / \mathrm{z}=303[\mathrm{M}+1]^{+}$.<smiles>CC(=O)CC([18F])c1cccs1</smiles>

\section{Chemical Formula: $\mathrm{C}_{15} \mathrm{H}_{16} \mathrm{O}_{3} \mathrm{~S}_{2}$ Mass: 308}

4-(thiophen-2-yl)-4-tosylbutan-2-one 3f ${ }^{12}$ white solid, mp: 107-109 ${ }^{\circ} \mathrm{C}$, yield 95\%, $293 \mathrm{mg} .{ }^{1} \mathrm{H}$ NMR $\left(\mathrm{CDCl}_{3}, 500 \mathrm{MHz}\right) \delta 2.17(\mathrm{~s}, 3 \mathrm{H}), 2.39(\mathrm{~s}, 3 \mathrm{H}), 3.19-3.25(\mathrm{~m}, 1 \mathrm{H}), 3.54-3.58(\mathrm{~m}, 1 \mathrm{H})$, 4.97-5.00 (m, 1H), $6.80(\mathrm{~s}, 1 \mathrm{H}), 6.81-6.86(\mathrm{~m}, 1 \mathrm{H}), 7.19-7.22(\mathrm{~m}, 3 \mathrm{H}), 7.45-7.46(\mathrm{~d}, J=8.5 \mathrm{~Hz}$, 2H). ${ }^{13} \mathrm{C} \mathrm{NMR}\left(\mathrm{CDCl}_{3}, 125 \mathrm{MHz}\right) \delta 21.8,30.6,43.0,61.9,127.0,129.2,129.6,133.6,134.6$, 145.1, 203.0. ESI-MS: $\mathrm{m} / \mathrm{z}=309[\mathrm{M}+1]^{+}$.<smiles>CC(=O)CC([As])c1ccco1</smiles>

\section{Chemical Formula: $\mathrm{C}_{15} \mathrm{H}_{16} \mathrm{O}_{4} \mathrm{~S}$ \\ Mass: 292}

4-(Furan-2-yl)-4-tosylbutan-2-one 3g, ${ }^{13}$ white solid, mp: 103-105 ${ }^{\circ} \mathrm{C}$, yield 93\%, $272 \mathrm{mg} .{ }^{1} \mathrm{H}$ NMR $\left(\mathrm{CDCl}_{3}, 500 \mathrm{MHz}\right) \delta 2.18(\mathrm{~s}, 3 \mathrm{H}), 2.41(\mathrm{~s}, 3 \mathrm{H}), 3.24-3.30(\mathrm{~m}, 1 \mathrm{H}), 3.41-3.45(\mathrm{~m}, 1 \mathrm{H})$, 4.84-4.87 (m, 1H), 6.16-6.17 (d, $J=3.0 \mathrm{~Hz}, 1 \mathrm{H}), 6.27(\mathrm{~s}, 1 \mathrm{H}), 7.23-7.25(\mathrm{~d}, J=8.0 \mathrm{~Hz}, 2 \mathrm{H})$, 7.46-7.48 (d, $J=8.0 \mathrm{~Hz}, 2 \mathrm{H}) .{ }^{13} \mathrm{C} \mathrm{NMR}\left(\mathrm{CDCl}_{3}, 125 \mathrm{MHz}\right) \delta 21.8,30.3,40.0,60.6,111.1,111.5$, 129.1, 129.1, 129.7, 134.1, 143.4, 145.2, 145.8, 203.0. ESI-MS: $\mathrm{m} / \mathrm{z}=293[\mathrm{M}+1]^{+}$.<smiles>[3H]C(C)CC(C)=O</smiles>

\section{Chemical Formula: $\mathrm{C}_{12} \mathrm{H}_{16} \mathrm{O}_{3} \mathrm{~S}$ Mass: 240}

4-Tosylpentan-2-one $3 \mathbf{h},{ }^{10}$ white solid, mp 80-82 (lit. 79-80 ${ }^{\circ} \mathrm{C}$ ), yield 73\%, $175 \mathrm{mg} .{ }^{1} \mathrm{H}$ NMR $\left(\mathrm{CDCl}_{3}, 500 \mathrm{MHz}\right) \delta 1.20-1.21(\mathrm{~d}, J=6.5 \mathrm{~Hz}, 3 \mathrm{H}), 2.17(\mathrm{~s}, 3 \mathrm{H}), 2.44(\mathrm{~s}, 3 \mathrm{H}), 2.53-2.59(\mathrm{~m}, 1 \mathrm{H})$, 3.15-3.20 (m, 1H), 3.58-3.65 (m, 1H), 7.34-7.36 (d, $J=8.0 \mathrm{~Hz}, 2 \mathrm{H}), 7.73-7.75(\mathrm{~d}, J=8.0 \mathrm{~Hz} .2 \mathrm{H})$. ${ }^{13} \mathrm{C} \mathrm{NMR}\left(\mathrm{CDCl}_{3}, 125 \mathrm{MHz}\right) \delta 14.3,21.7,30.5,42.6,55.6,129.0,130.0,134.1,145.0,204.2$. ESI-MS: $\mathrm{m} / \mathrm{z}=241[\mathrm{M}+1]^{+}$.<smiles>CN(C)C(=O)CCS</smiles>

\section{Chemical Formula: $\mathrm{C}_{12} \mathrm{H}_{17} \mathrm{NO}_{3} \mathrm{~S}$ Mass: 255}

$\mathrm{N}, \mathrm{N}$-Dimethyl-3-tosylpropanamide $3 \mathbf{3 i},{ }^{14}$ white solid, mp 97-99 ${ }^{\circ} \mathrm{C}$, yield 77\%, $196 \mathrm{mg} .{ }^{1} \mathrm{H}$ NMR $\left(\mathrm{CDCl}_{3}, 500 \mathrm{MHz}\right) \delta 2.45(\mathrm{~s}, 3 \mathrm{H}), 2.78-2.81(\mathrm{t}, J=7.0 \mathrm{~Hz}, 2 \mathrm{H}), 2.90(\mathrm{~s}, 3 \mathrm{H}), 3.00(\mathrm{~s}, 3 \mathrm{H})$, 3.43-3.46 (t, $J=7.0 \mathrm{~Hz}, 2 \mathrm{H}), 7.35-7.37(\mathrm{~d}, J=8.0 \mathrm{~Hz}, 2 \mathrm{H}), 7.78-7.80(\mathrm{~d}, J=8.0 \mathrm{~Hz}, 2 \mathrm{H}) .{ }^{13} \mathrm{C}$ NMR $\left(\mathrm{CDCl}_{3}, 125 \mathrm{MHz}\right) \delta 21.8,26.4,26.7,35.7,37.2,52.3,128.1,130.1,136.3,145.0,168.9$. ESI-MS: $\mathrm{m} / \mathrm{z}=256[\mathrm{M}+1]^{+}$. 
$\prod_{\mathrm{O}}^{\text {Ts }}$ OEt Chemical Formula: $\mathrm{C}_{12} \mathrm{H}_{16} \mathrm{O}_{4} \mathrm{~S}$

Ethyl 3-tosylpropanoate 3j, ${ }^{15}$ colourless oil, yield 94\%, $241 \mathrm{mg} .{ }^{1} \mathrm{H}$ NMR $\left(\mathrm{CDCl}_{3}, 500 \mathrm{MHz}\right) \delta$ $1.20-1.22(\mathrm{~m}, 3 \mathrm{H}), 21.44$ (s, 3H), 2.70-2.73 (t, $J=7.5 \mathrm{~Hz}, 2 \mathrm{H}), 3.38-3.41(\mathrm{t}, J=7.5 \mathrm{~Hz}, 2 \mathrm{H})$, 4.05-4.10 (q, $J=7.5 \mathrm{~Hz}, 2 \mathrm{H}), 7.35-7.37(\mathrm{~d}, J=8.0 \mathrm{~Hz}, 2 \mathrm{H}), 7.77-7.78(\mathrm{~d}, J=7.5 \mathrm{~Hz}, 2 \mathrm{H}) .{ }^{13} \mathrm{C}$ $\mathrm{NMR}\left(\mathrm{CDCl}_{3}, 125 \mathrm{MHz}\right) \delta 14.2,21.7,28.1,51.7,61.4,128.3,130.1,135.7,145.2,170.1$. ESI-MS: $\mathrm{m} / \mathrm{z}=257[\mathrm{M}+1]^{+}$.

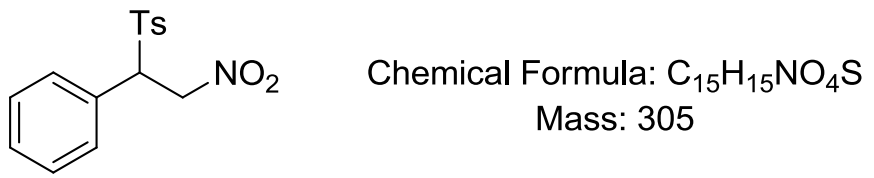

1-Methyl-4-((2-nitro-1-phenylethyl)sulfonyl)benzene $3 \mathbf{k},{ }^{16}$ white solid, $144-146{ }^{\circ} \mathrm{C}$ (lit. 147.5-148 $\left.{ }^{\circ} \mathrm{C}\right)$, yield 99\%, $302 \mathrm{mg} .{ }^{1} \mathrm{H}$ NMR $\left(\mathrm{CDCl}_{3}, 500 \mathrm{MHz}\right) \delta 2.41(\mathrm{~s}, 3 \mathrm{H}), 4.95-4.98(\mathrm{~m}, 1 \mathrm{H}), 5.05-5.09$ $(\mathrm{m}, 1 \mathrm{H}), 5.32-5.35(\mathrm{~m}, 1 \mathrm{H}), 7.14-7.15(\mathrm{~d}, J=7.0 \mathrm{~Hz}, 2 \mathrm{H}), 7.21-7.23(\mathrm{~d}, J=8.5 \mathrm{~Hz}, 2 \mathrm{H}), 7.26-7.36$ $(\mathrm{m}, 3 \mathrm{H}), 7.41-7.42(\mathrm{~d}, J=8.5 \mathrm{~Hz}, 2 \mathrm{H}) .{ }^{13} \mathrm{C} \mathrm{NMR}\left(\mathrm{CDCl}_{3}, 125 \mathrm{MHz}\right) \delta 21.8,68.1,73.0,129.1$, 129.3, 129.6, 129.9, 130.0, 133.1, 145.9. ESI-MS: $\mathrm{m} / \mathrm{z}=306[\mathrm{M}+1]^{+}$.

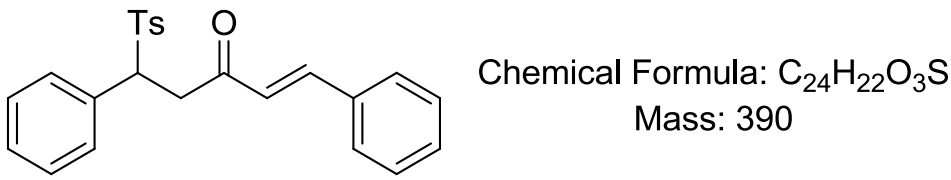

(E)-1,5-diphenyl-5-tosylpent-1-en-3-one 31, ${ }^{17}$ white solid, $174-176{ }^{\circ} \mathrm{C}$ (lit. 177-178 ${ }^{\circ} \mathrm{C}$ ), yield $98 \%$, $382 \mathrm{mg} .{ }^{1} \mathrm{H}$ NMR $\left(\mathrm{CDCl}_{3}, 500 \mathrm{MHz}\right) \delta 2.38(\mathrm{~s}, 3 \mathrm{H}), 3.58-3.64(\mathrm{~m}, 1 \mathrm{H}), 3.78-3.83(\mathrm{~m}, 1 \mathrm{H})$, 4.83-4.85 (m, 1H), 6.67-6.70 (d, $J=16.0 \mathrm{~Hz}, 1 \mathrm{H}), 7.17-7.26(\mathrm{~m}, 7 \mathrm{H}), 7.39-7.43(\mathrm{~m}, 5 \mathrm{H})$,

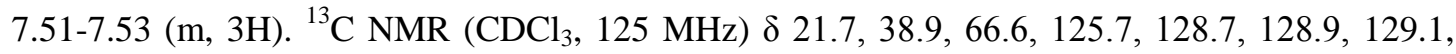
$129.5,129.9,131.0,132.7,134.2,144.1,144.9,194.9$. ESI-MS: $\mathrm{m} / \mathrm{z}=391[\mathrm{M}+1]^{+}$.<smiles>CN1C(=O)CC([TeH])C1=O</smiles>

Chemical Formula: $\mathrm{C}_{12} \mathrm{H}_{13} \mathrm{NO}_{4} \mathrm{~S}$

Mass: 267

1-Methyl-3-tosylpyrrolidine-2,5-dione 3m, ${ }^{18}$ white solid, $149-151{ }^{\circ} \mathrm{C}$, yield 75\%, $200 \mathrm{mg} .{ }^{1} \mathrm{H}$ NMR $\left(\mathrm{CDCl}_{3}, 500 \mathrm{MHz}\right) \delta 2.48(\mathrm{~s}, 3 \mathrm{H}), 2.95(\mathrm{~s}, 3 \mathrm{H}), 3.02-3.07(\mathrm{~m}, 1 \mathrm{H}), 3.30-3.34(\mathrm{~m}, 1 \mathrm{H})$, 4.30-4.33 (m, 1H), 7.40-7.41 (d, $J=8.5 \mathrm{~Hz}, 2 \mathrm{H}), 7.79-7.81(\mathrm{~d}, J=8.0 \mathrm{~Hz}, 2 \mathrm{H}) .{ }^{13} \mathrm{C} \mathrm{NMR}\left(\mathrm{CDCl}_{3}\right.$, $125 \mathrm{MHz}) \delta 21.9,25.6,30.0,63.5,129.5,130.2,133.7,146.4,169.7,173.1 . \mathrm{ESI}-\mathrm{MS}: \mathrm{m} / \mathrm{z}=268$ $[\mathrm{M}+1]^{+}$.<smiles>CCN1C(=O)CC([Hg])C1=O</smiles>

\section{Chemical Formula: $\mathrm{C}_{13} \mathrm{H}_{15} \mathrm{NO}_{4} \mathrm{~S}$}

Exact Mass: 281.0722

Elemental Analysis: C, 55.50; $\mathrm{H}, 5.37 ; \mathrm{N}$,

4.98; O, 22.75; S, 11.40

1-Ethyl-3-tosylpyrrolidine-2,5-dione 3n, white solid, mp: $155-157{ }^{\circ} \mathrm{C}$, yield $88 \%, 247 \mathrm{mg} .{ }^{1} \mathrm{H}$ NMR $\left(\mathrm{CDCl}_{3}, 500 \mathrm{MHz}\right) \delta 1.05-1.08(\mathrm{t}, J=7.5 \mathrm{~Hz}, 3 \mathrm{H}), 2.47(\mathrm{~s}, 3 \mathrm{H}), 3.01-3.06(\mathrm{~m}, 1 \mathrm{H})$, 3.27-3.32 (m, 1H), 3.49-3.52 (q, $J=7.0 \mathrm{~Hz}, 2 \mathrm{H}), 4.29-4.31(\mathrm{~m}, 1 \mathrm{H}), 7.39-7.41(\mathrm{~d}, J=8.0 \mathrm{~Hz}, 2 \mathrm{H})$, 7.79-7.80 (d, $J=8.0 \mathrm{~Hz}, 2 \mathrm{H}) .{ }^{13} \mathrm{C} \mathrm{NMR}\left(\mathrm{CDCl}_{3}, 125 \mathrm{MHz}\right) \delta 12.7,21.9,30.1,34.6,63.6,129.5$, 130.2, 133.6, 146.4, 168.6, 172.9. ESI-MS: $\mathrm{m} / \mathrm{z}=282[\mathrm{M}+1]^{+}$. Anal. Calcd for $\mathrm{C}_{13} \mathrm{H}_{15} \mathrm{NO}_{4} \mathrm{~S}: \mathrm{C}$, 
$55.50 \%$; H, 5.37\%; N, 4.98\%. Found: C, 55.63\%; H, 5.14\%; N, 5.07\%.<smiles>O=C1CC([18F])C(=O)N1c1ccccc1</smiles>

Chemical Formula: $\mathrm{C}_{17} \mathrm{H}_{15} \mathrm{NO}_{4} \mathrm{~S}$

Mass: 329

1-Phenyl-3-tosylpyrrolidine-2,5-dione 3o, ${ }^{19}$ white solid, mp: $175-177{ }^{\circ} \mathrm{C}$ (lit. 176-177 ${ }^{\circ} \mathrm{C}$ ), yield 97\%, $317 \mathrm{mg} .{ }^{1} \mathrm{H}$ NMR $\left(\mathrm{CDCl}_{3}, 500 \mathrm{MHz}\right) \delta 2.45(\mathrm{~s}, 3 \mathrm{H}), 3.18-3.23(\mathrm{~m}, 1 \mathrm{H}), 3.43-3.48(\mathrm{~m}, 1 \mathrm{H})$, 4.45-4.48 (m, 1H), 7.12-7.13 (d, $J=7.0 \mathrm{~Hz}, 1 \mathrm{H}), 7.37-7.44(\mathrm{~m}, 5 \mathrm{H}), 7.81-7.82(\mathrm{~d}, J=8.0 \mathrm{~Hz}, 2 \mathrm{H})$. ${ }^{13} \mathrm{C}$ NMR $\left(\mathrm{CDCl}_{3}, 125 \mathrm{MHz}\right) \delta 21.9,30.3,63.8,126.5,129.3,129.4,130.3,131.2,133.5,146.5$, 168.0, 172.2. ESI-MS: $\mathrm{m} / \mathrm{z}=330[\mathrm{M}+1]^{+}$.<smiles>O=C1CC([As])C(=O)N1Cc1ccccc1</smiles>

\section{Chemical Formula: $\mathrm{C}_{18} \mathrm{H}_{17} \mathrm{NO}_{4} \mathrm{~S}$ Exact Mass: 343.0878} Elemental Analysis: C, 62.96; $\mathrm{H}, 4.99$; $\mathrm{N}, 4.08 ; \mathrm{O}, 18.64 ; \mathrm{S}, 9.34$

1-Benzyl-3-tosylpyrrolidine-2,5-dione 3p, white solid, mp: 180-182 ${ }^{\circ} \mathrm{C}$, yield 83\%, $285 \mathrm{mg} .{ }^{1} \mathrm{H}$ NMR $\left(\mathrm{CDCl}_{3}, 500 \mathrm{MHz}\right) \delta 2.44(\mathrm{~s}, 3 \mathrm{H}), 3.01-3.07(\mathrm{~m}, 1 \mathrm{H}), 3.29-3.33(\mathrm{~m}, 1 \mathrm{H}), 4.28-4.31(\mathrm{~m}, 1 \mathrm{H})$, $4.60(\mathrm{~s}, 2 \mathrm{H}), 7.27-7.31(\mathrm{~m}, 7 \mathrm{H}), 7.70-7.72(\mathrm{~d}, J=8.5 \mathrm{~Hz}, 2 \mathrm{H}) .{ }^{13} \mathrm{C} \mathrm{NMR}\left(\mathrm{CDCl}_{3}, 125 \mathrm{MHz}\right) \delta$ 21.9, 30.1, 43.2, 63.5, 128.3, 128.8, 129.5, 130.1, 133.4, 134.9, 146.3, 168.5, 172.7. ESI-MS: m/z $=344[\mathrm{M}+1]^{+}$. Anal. Calcd for $\mathrm{C}_{18} \mathrm{H}_{17} \mathrm{NO}_{4} \mathrm{~S}: \mathrm{C}, 62.96 \% ; \mathrm{H}, 4.99 \% ; \mathrm{N}, 4.08 \%$. Found: $\mathrm{C}, 62.71 \%$; $\mathrm{H}, 5.29 \%$; N, 3.84\%.<smiles>O=C(CC(c1ccccc1)S(=O)(=O)c1ccc(Cl)cc1)c1ccccc1</smiles>

\section{Chemical Formula: $\mathrm{C}_{21} \mathrm{H}_{17} \mathrm{ClO}_{3} \mathrm{~S}$ Mass: 384}

3-((4-Chlorophenyl)sulfonyl)-1,3-diphenylpropan-1-one $\mathbf{3 q}{ }^{20}$ white solid, mp: $172-174{ }^{\circ} \mathrm{C}$ $\left(175-176{ }^{\circ} \mathrm{C}\right)$, yield $99 \%, 380 \mathrm{mg} .{ }^{1} \mathrm{H}$ NMR $\left(\mathrm{CDCl}_{3}, 500 \mathrm{MHz}\right) \delta 3.91-3.96(\mathrm{~m}, 1 \mathrm{H}), 4.12-4.16(\mathrm{~m}$, $1 \mathrm{H}), 4.93-4.96(\mathrm{dd}, J=9.5,3.5 \mathrm{~Hz}, 1 \mathrm{H}), 7.20-7.27(\mathrm{~m}, 5 \mathrm{H}), 7.35-7.36(\mathrm{t}, J=7.5 \mathrm{~Hz}, 2 \mathrm{H})$, 7.45-7.49 (m, 4H), 7.59-7.61 (t, $J=7.5 \mathrm{~Hz}, 1 \mathrm{H}), 7.94-7.96(\mathrm{~d}, J=7.5 \mathrm{~Hz}, 2 \mathrm{H}) .{ }^{13} \mathrm{C} \mathrm{NMR}\left(\mathrm{CDCl}_{3}\right.$, $125 \mathrm{MHz}) \delta 36.9,66.8,128.3,128.8,128.9,129.2,129.9,130.6,132.5,133.9,135.6,136.2,140.7$, 194.8. ESI-MS: $\mathrm{m} / \mathrm{z}=385[\mathrm{M}+1]^{+}$.

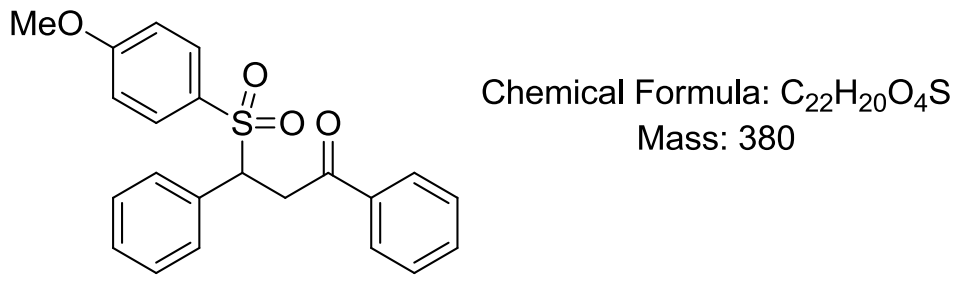

3-((4-methoxyphenyl)sulfonyl)-1,3-diphenylpropan-1-one 3r, ${ }^{11}$ white solid, mp 158-160 ${ }^{\circ} \mathrm{C}$ (lit. $\left.162-163{ }^{\circ} \mathrm{C}\right)$, yield 86\%, $327 \mathrm{mg} .{ }^{1} \mathrm{H}$ NMR $\left(\mathrm{CDCl}_{3}, 500 \mathrm{MHz}\right) \delta 3.84(\mathrm{~s}, 1 \mathrm{H}), 3.90-3.96(\mathrm{~m}, 1 \mathrm{H})$, 4.11-4.15 (m, 1H), 4.89-4.92 (dd, $J=9.5,3.5 \mathrm{~Hz}, 1 \mathrm{H}), 6.83-6.85(\mathrm{~d}, J=9.0 \mathrm{~Hz}, 2 \mathrm{H}), 7.21-7.27(\mathrm{~m}$, $5 \mathrm{H}), 7.45-7.48(\mathrm{~m}, 4 \mathrm{H}), 7.57-7.60(\mathrm{t}, J=7.5 \mathrm{~Hz}, 1 \mathrm{H}), 7.94-7.96(\mathrm{~d}, J=7.0 \mathrm{~Hz}, 2 \mathrm{H}) .{ }^{13} \mathrm{C}$ NMR $\left(\mathrm{CDCl}_{3}, 125 \mathrm{MHz}\right) \delta 37.2,55.7,66.8,114.1,128.3,128.6,128.9,129.9,131.3,133.0,133.7$, 136.4, 163.9, 195.0. ESI-MS: $\mathrm{m} / \mathrm{z}=381[\mathrm{M}+1]^{+}$. 
<smiles>O=C(CC(c1ccccc1)S(=O)(=O)c1ccc(Br)cc1)c1ccccc1</smiles>

Chemical Formula: $\mathrm{C}_{21} \mathrm{H}_{17} \mathrm{BrO}_{3} \mathrm{~S}$

Exact Mass: 428.0082

Elemental Analysis: C, 58.75; H, 3.99;

$\mathrm{Br}, 18.61 ; \mathrm{O}, 11.18 ; \mathrm{S}, 7.47$

3-((4-bromophenyl)sulfonyl)-1,3-diphenylpropan-1-one 3s, white solid, mp: $178-180{ }^{\circ} \mathrm{C}$, yield 89\%, $381 \mathrm{mg} .{ }^{1} \mathrm{H}$ NMR $\left(\mathrm{CDCl}_{3}, 500 \mathrm{MHz}\right) \delta$ 3.91-3.96 (m, 1H), 4.12-4.16 (m, 1H), 4.93-5.07 (m, $1 \mathrm{H}), 7.20-7.28(\mathrm{~m}, 5 \mathrm{H}), 7.38-7.40(\mathrm{~d}, \mathrm{~J}=8.5 \mathrm{~Hz}, 2 \mathrm{H}), 7.48-7.49(\mathrm{t}, J=7.5 \mathrm{~Hz}, 1 \mathrm{H}), 7.52-7.53(\mathrm{~d}$, $J=8.5 \mathrm{~Hz}, 2 \mathrm{H}), 7.58-7.61(\mathrm{t}, J=7.5 \mathrm{~Hz}, 1 \mathrm{H}), 7.94-7.96(\mathrm{~d}, J=7.0 \mathrm{~Hz}, 2 \mathrm{H}) .{ }^{13} \mathrm{C} \mathrm{NMR}\left(\mathrm{CDCl}_{3}\right.$, $125 \mathrm{MHz}) \delta 36.9,66.7,128.7,128.8,128.9,129.2,129.3,129.9,130.6,132.2,132.5,133.9,136.2$, 194.8. ESI-MS: $\mathrm{m} / \mathrm{z}=429[\mathrm{M}+1]^{+}$. Anal. Calcd for $\mathrm{C}_{21} \mathrm{H}_{17} \mathrm{BrO}_{3} \mathrm{~S}: \mathrm{C}, 58.75 \%$; H, 3.99\%. Found: $\mathrm{C}$, $59.01 \%$; H, 3.87\%.<smiles>Cc1ccccc1S(=O)(=O)C(CC(=O)c1ccccc1)c1ccccc1</smiles>

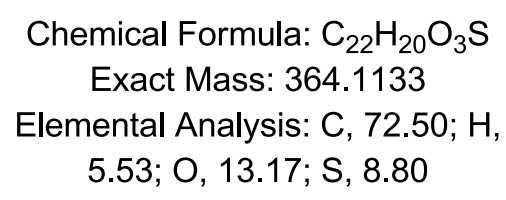

1,3-Diphenyl-3-(o-tolylsulfonyl)propan-1-one 3t, white solid, mp: $151-153{ }^{\circ} \mathrm{C}$, yield 81\%, $294 \mathrm{mg}$. ${ }^{1} \mathrm{H}$ NMR $\left(\mathrm{CDCl}_{3}, 500 \mathrm{MHz}\right) \delta 2.57(\mathrm{~s}, 3 \mathrm{H}), 3.91-3.96(\mathrm{~m}, 1 \mathrm{H}), 4.11-4.16(\mathrm{~m}, 1 \mathrm{H}), 5.01-5.10(\mathrm{~m}$, $1 \mathrm{H}), 7.13-7.16(\mathrm{t}, J=7.5 \mathrm{~Hz}, 1 \mathrm{H}), 7.19-7.26(\mathrm{~m}, 6 \mathrm{H}), 7.42-7.49(\mathrm{~m}, 3 \mathrm{H}), 7.59-7.61(\mathrm{~m}, 2 \mathrm{H})$, 7.94-7.96 (d, $J=7.5 \mathrm{~Hz}, 2 \mathrm{H}) .{ }^{13} \mathrm{C} \mathrm{NMR}\left(\mathrm{CDCl}_{3}, 125 \mathrm{MHz}\right) \delta 20.3,36.7,65.6,126.3,128.3,128.6$, 128.9, 129.1, 129.9, 131.4, 132.6, 133.8, 135.2, 136.3, 139.3, 195.2. ESI-MS: $\mathrm{m} / \mathrm{z}=365[\mathrm{M}+1]^{+}$. Anal. Calcd for $\mathrm{C}_{22} \mathrm{H}_{20} \mathrm{O}_{3} \mathrm{~S}: \mathrm{C}, 72.50 \% ; \mathrm{H}, 5.53 \%$. Found: C, 72.68\%; H, 5.46\%.<smiles>O=C(CC(c1ccccc1)S(=O)(=O)c1ccccc1)c1ccccc1</smiles>

\section{Chemical Formula: $\mathrm{C}_{21} \mathrm{H}_{18} \mathrm{O}_{3} \mathrm{~S}$ Mass: 350}

1,3-Diphenyl-3-(phenylsulfonyl)propan-1-one 3u, ${ }^{11}$ white solid, mp: $148-150{ }^{\circ} \mathrm{C}$ (lit. 152-153 ${ }^{\circ} \mathrm{C}$ ), yield 87\%, $304 \mathrm{mg} .{ }^{1} \mathrm{H}$ NMR $\left(\mathrm{CDCl}_{3}, 500 \mathrm{MHz}\right) \delta 3.92-3.98(\mathrm{~m}, 1 \mathrm{H}), 4.12-4.16(\mathrm{~m}, 1 \mathrm{H})$, 4.94-4.96 (m, 1H), 7.20-7.27 (m, 5H), 7.37-7.60 (m, 8H), 7.94-7.96 (d, $J=8.0 \mathrm{~Hz}, 2 \mathrm{H}) .{ }^{13} \mathrm{C} \mathrm{NMR}$ $\left(\mathrm{CDCl}_{3}, 125 \mathrm{MHz}\right) \delta 37.0,66.7,128.3,128.6,128.9,129.1,129.9,132.7,133.8,136.3,137.1$, 195.0. ESI-MS: $\mathrm{m} / \mathrm{z}=351[\mathrm{M}+1]^{+}$.<smiles>CC1(C)CCC(=O)CC1[18F]</smiles>

\section{Chemical Formula: $\mathrm{C}_{15} \mathrm{H}_{20} \mathrm{O}_{3} \mathrm{~S}$ \\ Exact Mass: 280.1133}

Elemental Analysis: C, 64.26; H, 7.19; O, 17.12; S, 11.44

4,4-Dimethyl-3-tosylcyclohexanone 3v. white solid, mp: 121-123 ${ }^{\circ} \mathrm{C}$, yield 95\%, $266 \mathrm{mg} .{ }^{1} \mathrm{H}$ $\operatorname{NMR}\left(\mathrm{CDCl}_{3}, 500 \mathrm{MHz}\right) \delta 1.47(\mathrm{~s}, 6 \mathrm{H}), 1.61-1.68(\mathrm{~m}, 1 \mathrm{H}), 1.78-1.83(\mathrm{~m}, 1 \mathrm{H}), 2.21-2.28(\mathrm{~m}, 2 \mathrm{H})$, 2.37-2.51 (m, 4H), 2.68-2.73 (m, 1H), 3.11-3.14 (m, 1H), $7.31(\mathrm{~d}, J=8.0 \mathrm{~Hz}, 2 \mathrm{H}), 7.68(\mathrm{~d}, J=8.5$ $\mathrm{Hz}, 2 \mathrm{H}) .{ }^{13} \mathrm{C} \mathrm{NMR}\left(\mathrm{CDCl}_{3}, 125 \mathrm{MHz}\right) \delta 21.4,21.8,30.2,34.9,37.4,39.1,41.0,69.2,128.6,130.1$, 136.2, 145.1, 207.5. ESI-MS: $\mathrm{m} / \mathrm{z}=281[\mathrm{M}+1]^{+}$. Anal. Calcd for $\mathrm{C}_{15} \mathrm{H}_{20} \mathrm{O}_{3} \mathrm{~S}: \mathrm{C}, 64.26 \% ; \mathrm{H}$, 7.19\%. Found: C, $63.97 \%$; H, 7.35\%. 


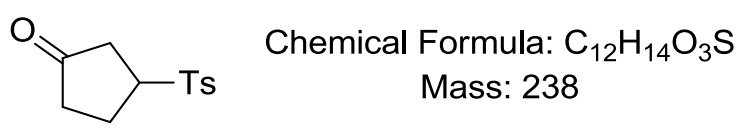

3-Tosylcyclopentanone $3 w^{21}$ white solid, mp: $68-70{ }^{\circ} \mathrm{C}$ (lit. 73-74,5 ${ }^{\circ} \mathrm{C}$ ), yield 82\%, $195 \mathrm{mg} .{ }^{1} \mathrm{H}$ $\mathrm{NMR}\left(\mathrm{CDCl}_{3}, 500 \mathrm{MHz}\right) \delta$ 2.20-2.30 (m, 2H), 2.38-2.54 (m, 6H), 2.63-2.69 (m, 1H), 3.71-3.77 $(\mathrm{m}, 1 \mathrm{H}), 7.38(\mathrm{~d}, J=8.0 \mathrm{~Hz}, 2 \mathrm{H}), 7.77(\mathrm{~d}, J=8.0 \mathrm{~Hz}, 2 \mathrm{H}) .{ }^{13} \mathrm{C} \mathrm{NMR}\left(\mathrm{CDCl}_{3}, 125 \mathrm{MHz}\right) \delta 21.8$, 23.3, 37.2, 38.8, 60.9, 128.8, 130.3, 134.5, 145.5, 213.1. ESI-MS: $\mathrm{m} / \mathrm{z}=239[\mathrm{M}+1]^{+}$.

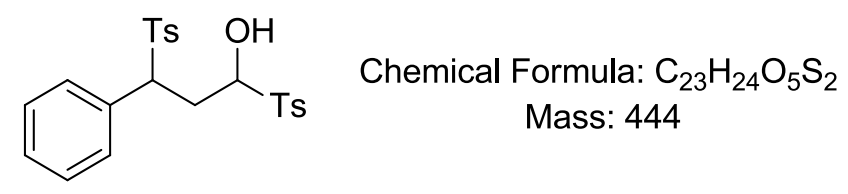

3-Phenyl-1,3-ditosylpropan-1-ol 4, ${ }^{22}$ white solid, mp: $130-132{ }^{\circ} \mathrm{C}$ (lit. 128-129 ${ }^{\circ} \mathrm{C}$ ), yield $72 \%$, $320 \mathrm{mg} .{ }^{1} \mathrm{H}$ NMR (DMSO-d 6 , $\left.500 \mathrm{MHz}\right) \delta 2.04-2.08(\mathrm{t}, J=12.0 \mathrm{~Hz}, 1 \mathrm{H}), 2.33(\mathrm{~s}, 3 \mathrm{H}), 2.38(\mathrm{~s}$, $3 \mathrm{H}), 2.53-2.59(\mathrm{t}, J=12.5 \mathrm{~Hz}, 1 \mathrm{H}), 3.85-3.87(\mathrm{~d}, J=11.0 \mathrm{~Hz}, 1 \mathrm{H}), 4.38-4.40(\mathrm{~d}, J=12.0 \mathrm{~Hz}, 1 \mathrm{H})$, 7.09-7.10 (d, $J=7.0 \mathrm{~Hz}, 2 \mathrm{H}), 7.29-7.44(\mathrm{~m}, 9 \mathrm{H}), 7.58-7.59(\mathrm{~d}, J=7.0 \mathrm{~Hz}, 2 \mathrm{H}) .{ }^{13} \mathrm{C}$ NMR $\left(\right.$ DMSO-d $\left._{6}, 125 \mathrm{MHz}\right) \delta 21.6,28.7,66.2,85.8,129.2$ (4C), 129.3, 129.9, 130.2, 130.4 (4C), 130.7, 132.8, 134.4, 145.2, 145.4. ESI-MS: $\mathrm{m} / \mathrm{z}=445[\mathrm{M}+1]^{+}$.

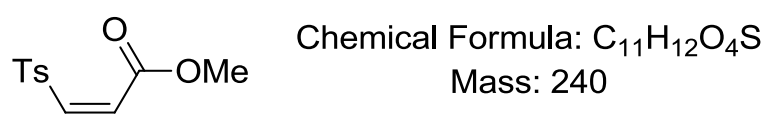

(Z)-methyl 3-tosylacrylate 5, ${ }^{23}$ light yellow oil, yield 98\%, $235 \mathrm{mg} .{ }^{1} \mathrm{H} \mathrm{NMR}\left(\mathrm{CDCl}_{3}, 500 \mathrm{MHz}\right) \delta$ $2.44(\mathrm{~s}, 3 \mathrm{H}), 3.88(\mathrm{~s}, 3 \mathrm{H}), 6.46-6.49(\mathrm{~d}, J=11.5 \mathrm{~Hz}, 1 \mathrm{H}), 6.53-6.55(\mathrm{~d}, J=11.5 \mathrm{~Hz}, 1 \mathrm{H})$, 7.35-7.36 (d, $J=8.0 \mathrm{~Hz}, 2 \mathrm{H}), 7.84-7.86(\mathrm{~d}, J=8.0 \mathrm{~Hz}, 2 \mathrm{H}) .{ }^{13} \mathrm{C} \mathrm{NMR}\left(\mathrm{CDCl}_{3}, 125 \mathrm{MHz}\right) \delta 21.8$, $52.9,128.4,130.1,131.2,136.0,136.5,145.4,164.6$. ESI-MS: $\mathrm{m} / \mathrm{z}=241[\mathrm{M}+1]^{+}$.<smiles>O=C1CCCC(O)=C1C(F)c1ccccc1</smiles>

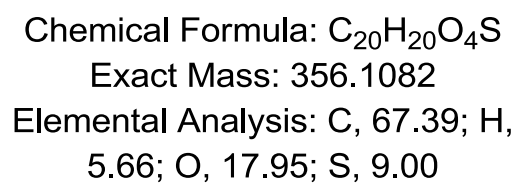

3-hydroxy-2-(phenyl(tosyl)methyl)cyclohex-2-enone 6a, white solid, mp: 240-242 ${ }^{\circ} \mathrm{C}$, yield 99\%, $352 \mathrm{mg} .{ }^{1} \mathrm{H}$ NMR $\left(\mathrm{CDCl}_{3}, 500 \mathrm{MHz}\right) \delta 1.84-1.87(\mathrm{~m}, 2 \mathrm{H}), 2.05-2.12(\mathrm{~m}, 1 \mathrm{H}), 2.30-2.34(\mathrm{~m}, 1 \mathrm{H})$, 2.30-2.34 (m, 1H), $2.43(\mathrm{~s}, 3 \mathrm{H}), 2.54-2.64(\mathrm{~m}, 2 \mathrm{H}), 6.26(\mathrm{~s}, 1 \mathrm{H}), 7.27-7.33(\mathrm{~m}, 5 \mathrm{H}), 7.40-7.41(\mathrm{t}, \mathrm{J}$ $=6.5 \mathrm{~Hz}, 2 \mathrm{H}), 7.63-7.65(\mathrm{~d}, \mathrm{~J}=8.0 \mathrm{~Hz}, 2 \mathrm{H}), 10.44(\mathrm{~s}, 1 \mathrm{H}) \cdot{ }^{13} \mathrm{C} \mathrm{NMR}\left(\mathrm{CDCl}_{3}, 125 \mathrm{MHz}\right) \delta 19.9$, 21.8, 31.0, 36.1, 66.1, 109.2, 128.7, 128.8, 129.0, 129.7, 130.3, 131.3, 133.8, 145.6, 176.3, 195.9. ESI-MS: $\mathrm{m} / \mathrm{z}=357[\mathrm{M}+1]^{+}$. Anal. Calcd for $\mathrm{C}_{20} \mathrm{H}_{20} \mathrm{O}_{4} \mathrm{~S}: \mathrm{C}, 67.39 \%$; $\mathrm{H}, 5.66 \%$. Found: C, 67.18\%; $\mathrm{H}, 5.96 \%$.<smiles>CC1(C)CC(=O)C(C([As])c2ccccc2)=C(O)C1</smiles>

\section{Chemical Formula: $\mathrm{C}_{22} \mathrm{H}_{24} \mathrm{O}_{4} \mathrm{~S}$ \\ Exact Mass: 384.1395}

Elemental Analysis: C, 68.72; H, 6.29;

O, 16.64; S, 8.34

3-hydroxy-5,5-dimethyl-2-(phenyl(tosyl)methyl)cyclohex-2-enone $\mathbf{6 b}$, white solid, mp > $250{ }^{\circ} \mathrm{C}$, 
89\%, $342 \mathrm{mg} .{ }^{1} \mathrm{H}$ NMR $\left(\mathrm{CDCl}_{3}, 500 \mathrm{MHz}\right) \delta 0.86(\mathrm{~s}, 3 \mathrm{H}), 0.94(\mathrm{~s}, 3 \mathrm{H}), 2.02-2.05(\mathrm{~d}, J=16.0 \mathrm{~Hz}$, $1 \mathrm{H}), 2.12-2.16(\mathrm{~d}, J=16.0 \mathrm{~Hz}, 1 \mathrm{H}), 2.38-2.49(\mathrm{~m}, 5 \mathrm{H}), 6.24(\mathrm{~s}, 1 \mathrm{H}), 7.26-7.33(\mathrm{~m}, 5 \mathrm{H}), 7.39-7.41$ $(\mathrm{m}, 2 \mathrm{H}), 7.65-7.67(\mathrm{~d}, J=8.0 \mathrm{~Hz}, 2 \mathrm{H}), 10.41(\mathrm{~s}, 2 \mathrm{H}) .{ }^{13} \mathrm{C} \mathrm{NMR}\left(\mathrm{CDCl}_{3}, 125 \mathrm{MHz}\right) \delta 21.8,27.6$, 28.3, 31.3, 31.5, 44.5, 49.9, 66.1, 108.0, 128.3, 128.6, 128.9, 129.0, 129.8, 130.3, 131.1, 134.0, 145.5, 174.4, 195.8. ESI-MS: $\mathrm{m} / \mathrm{z}=385[\mathrm{M}+1]^{+}$. Anal. Calcd for $\mathrm{C}_{22} \mathrm{H}_{24} \mathrm{O}_{4} \mathrm{~S}: \mathrm{C}, 68.72 \% ; \mathrm{H}$, 6.29\%. Found: C, $69.01 \%$; H, 5.99\%.<smiles>O=C(NC([13F])c1ccccc1)c1ccccc1</smiles>

\section{Chemical Formula: $\mathrm{C}_{21} \mathrm{H}_{19} \mathrm{NO}_{3} \mathrm{~S}$} Mass: 365

$N$-(phenyl(tosyl)methyl)benzamide 7a,${ }^{24}$ white solid, mp: > $250{ }^{\circ} \mathrm{C}$, yield $71 \%, 259 \mathrm{mg} .{ }^{1} \mathrm{H}$ NMR $\left(\mathrm{CDCl}_{3} / \mathrm{DMSO}_{-} \mathrm{d}_{6}, 500 \mathrm{MHz}\right) \delta 2.34(\mathrm{~s}, 3 \mathrm{H}), 6.52-6.54(\mathrm{~d}, J=10.0 \mathrm{~Hz}, 1 \mathrm{H}), 7.29-7.37(\mathrm{~m}, 7 \mathrm{H})$, 7.45-7.48 (d, $J=7.5 \mathrm{~Hz}, 1 \mathrm{H}), 7.65-7.71(\mathrm{~m}, 6 \mathrm{H}), 9.62-9.64(\mathrm{~d}, J=10.0 \mathrm{~Hz}, 1 \mathrm{H}) .{ }^{13} \mathrm{C} \mathrm{NMR}$ $\left(\mathrm{CDCl}_{3} / \mathrm{DMSO}_{6} \mathrm{~d}_{6}, 125 \mathrm{MHz}\right) \delta 21.7,73.4,128.4,128.6,129.5,129.7,129.9,130.4,131.0,132.0$, 133.8, 134.5, 145.0, 167.3. ESI-MS: $\mathrm{m} / \mathrm{z}=366[\mathrm{M}+1]^{+}$.<smiles>CN(C)c1ccc(C([As])NC(=O)c2ccccc2)cc1</smiles>

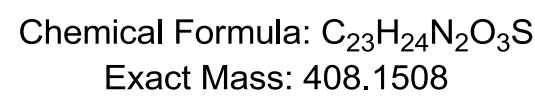

$\mathrm{N}$-((4-(dimethylamino)phenyl)(tosyl)methyl)benzamide $7 \mathbf{b}$, white solid, mp: $>250{ }^{\circ} \mathrm{C}$, yield $88 \%$, $360 \mathrm{mg} .{ }^{1} \mathrm{H}$ NMR (DMSO-d $\left.{ }_{6}, 500 \mathrm{MHz}\right) \delta 2.32(\mathrm{~s}, 3 \mathrm{H}), 2.89$ (s, 6H), 6.37-6.39 (d, $J=10.0 \mathrm{~Hz}$, $1 \mathrm{H}), 6.69-6.70(\mathrm{~d}, J=8.0 \mathrm{~Hz}, 2 \mathrm{H}), 7.32-7.41(\mathrm{~m}, 4 \mathrm{H}), 7.47-7.51(\mathrm{~m}, 3 \mathrm{H}), 7.61-7.71(\mathrm{~m}, 4 \mathrm{H})$, 9.53-9.55 (d, $J=10.5 \mathrm{~Hz}, 1 \mathrm{H}) .{ }^{13} \mathrm{C}$ NMR (DMSO-d 6 , $\left.125 \mathrm{MHz}\right) \delta 21.7,73.3,112.2,117.5,128.4$, 128.6, 129.5, 130.1, 131.4, 132.2, 134.0, 135.0, 144.9, 151.5, 167.1. ESI-MS: $\mathrm{m} / \mathrm{z}=409[\mathrm{M}+1]^{+}$. Anal. Calcd for $\mathrm{C}_{23} \mathrm{H}_{24} \mathrm{~N}_{2} \mathrm{O}_{3} \mathrm{~S}$ : C, $67.62 \%$; H, 5.92\%; N 6.86\%. Found: C, 67.45\%; H, 6.28\%; N $6.75 \%$.

[1] Frisch, M. J.; Trucks, G. W.; Schlegel, H. B.; Scuseria, G. E.; Robb, M. A.; Cheeseman, J. R.; Scalmani, G.; Barone, V.; Mennucci, B.; Petersson, G. Gaussian 09, Revision A.02; Gaussian: Wallingford, CT, USA, 2009.

[2] Marenich, A. V.; Cramer, C. J.; Truhlar, D. G. Universal Solvation Model Based on Solute Electron Density and on a Continuum Model of the Solvent Defined by the Bulk Dielectric Constant and Atomic Surface Tensions. J. Phys. Chem. B 2009, 113, 6378-6396.

[3] Zhou, B.-j.; Agarwal M.; Wong C. F. Variable atomic radii for continuum-solvent electrostatics calculation. J. Chem. Phys. 2008, 129, 014509.

[4] Maeda, S.; Saito, R.; Morokuma, K. Finding Minimum Structures on the Seam of Crossing in Reactions of Type $\mathrm{A}+\mathrm{B} \rightarrow \mathrm{X}$ : Exploration of Nonadiabatic Ignition Pathways of Unsaturated Hydrocarbons. J. Phys. Chem. Lett. 2011, 2, 852-857.

[5] Maeda, S.; Morokuma, K. Finding Reaction Pathways of Type A+B $\rightarrow$ X: Toward Systematic Prediction of Reaction Mechanisms. J. Chem. Theor. Comput. 2011, 7, 2335-2345.

[6] Maeda, S.; Ohno, K.; Morokuma, K. Systematic Exploration of the Mechanism of Chemical Reactions: The Global Reaction Route Mapping (GRRM) Strategy Using the ADDF and AFIR Methods. Phys. Chem. Chem. Phys. 2013, 15, 3683-3701. 
[7] Ohno, K.; Maeda, S. A scaled hypersphere search method for the topography of reaction pa1thways on the potential energy surface. Chem. Phys. Lett. 2004, 384, 277-282.

[8] Maeda, S.; Ohno, K. Global Mapping of Equilibrium and Transition Structures on Potential Energy Surfaces by the Scaled Hypersphere Search Method: Applications to ab Initio Surfaces of Formaldehyde and Propyne Molecules. J. Phys. Chem. A 2005, 109, 5742-5753.

[9] Ohno, K.; Maeda, S., Global Reaction Route Mapping on Potential Energy Surfaces of Formaldehyde, Formic Acid, and Their Metal-Substituted Analogues. J. Phys. Chem. A 2006, 110, 8933-8941.

[10] Sreedhar, B.; Reddy, M. A.; Reddy, P. S. FeCl3/TMSCl: An Effective Catalytic System for the Conjugate Addition of Sodium p-Toluenesulfinate to $\alpha, \beta$-Enones. Synlett 2008, 1949-1952.

[11] Jin, Z.; Xu, J.; Yang, S.; Song, B.-A.; Chi, Y. R. Enantioselective Sulfonation of Enones with Sulfonyl Imines by Cooperative N-Heterocyclic-Carbene/Thiourea/Tertiary-Amine Multicatalysis. Angew. Chem. Int. Ed. 2013, 52, 12354-12358.

[12] Messinger, P.; Gompertz, J. Sulfone als chemische Transportformen germicid wirkender Stoffe, 6. Mitt.: Darstellung von Sulfonyl-keton-Mannichbasen. Arch. Pharm. 1975, 308, 737-742. [13] Korikov, P. V.; Gerasimova, N. P.; Moskvichyov, Y. A.; Alov, E. M.; Nozhnin, N. A.; Shutova, I. V. Izvestiya Vysshikh Uchebnykh Zavedenii, Khimiya i Khimicheskaya Tekhnologiya 2000, 43, 64-68.

[14] Commercial chemical, CAS number: 694517-80-1.

[15] Yang, Y.; Tang, L.; Zhang, S.; Guo, X.; Zha, Z.; Wang, Z. Catalyst-free sulfonylation of activated alkenes for highly efficient synthesis of mono-substituted ethyl sulfones in water. Green Chem. 2014, 16, 4106-4109.

[16] Bilaya, E. E.; Obushak, N. D.; Buchinskii, A. M.; Ganushchak, N. I. Reaction of Arendiazonium Salts and SO2 with $\alpha$-Nitroolefins. Russ. J. Org. Chem. 2004, 40, 191-194.

[17] Henry, R. A.; Moore, D. W. Thermal decomposition of p-tosylhydrazones. J. Org. Chem. 1967, 32, 4145-4147.

[18] Dietz, H.-J.; Rieck, G.; Schulze, B. Synthese neuer Pyrrolidin-2,5-dione. Zeitschrift für Chemie 1989, 29, 284-285.

[19] Abramovitch, R. A.; Mathur, S. S.; Saunders, D. W.; Vanderpool, D. P. Pyridinium p-toluenesulfonylmethylide as a formyl anion equivalent. Tetrahedron Lett. 1980, 21, 705-708.

[20] Gilman, H.; Cason, L. F. Some Addition Reactions of Chalcones. I. The Preparation of Some $\gamma$-Ketosulfones. J. Am. Chem. Soc. 1950, 72, 3469-3472.

[21] Rothberg, I.; Sundoro, B.; Balanikas, G.; Kirsch, S. Preparation and stereochemistry of methylation of some cycloalkyl sulfones. J. Org. Chem. 1983, 48, 4345-4348.

[22] Janssen, C. G. M.; Van Lier, P. M.; Buck, H. M.; Godefroi, E. F. Synthesis of 2-[2-aryl-2-(p-toluenesulfonyl)ethyl]-1,3-dioxolanes. J. Org. Chem. 1979, 44, 4199-4202.

[23] Commercial chemical, CAS number: 114140-97-5.

[24] Morton, J.; Rahim, A.; Walker, E. R. H. A new general method of $\alpha$-amino-alkylation. Tetrahedron Lett. 1982, 23, 4123-4126. 
3 NMR Spectra of All Products

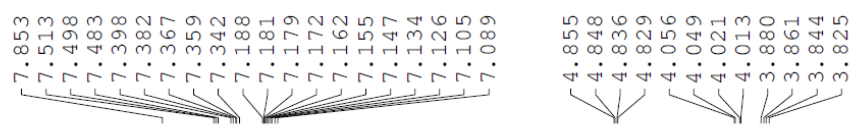

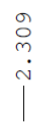

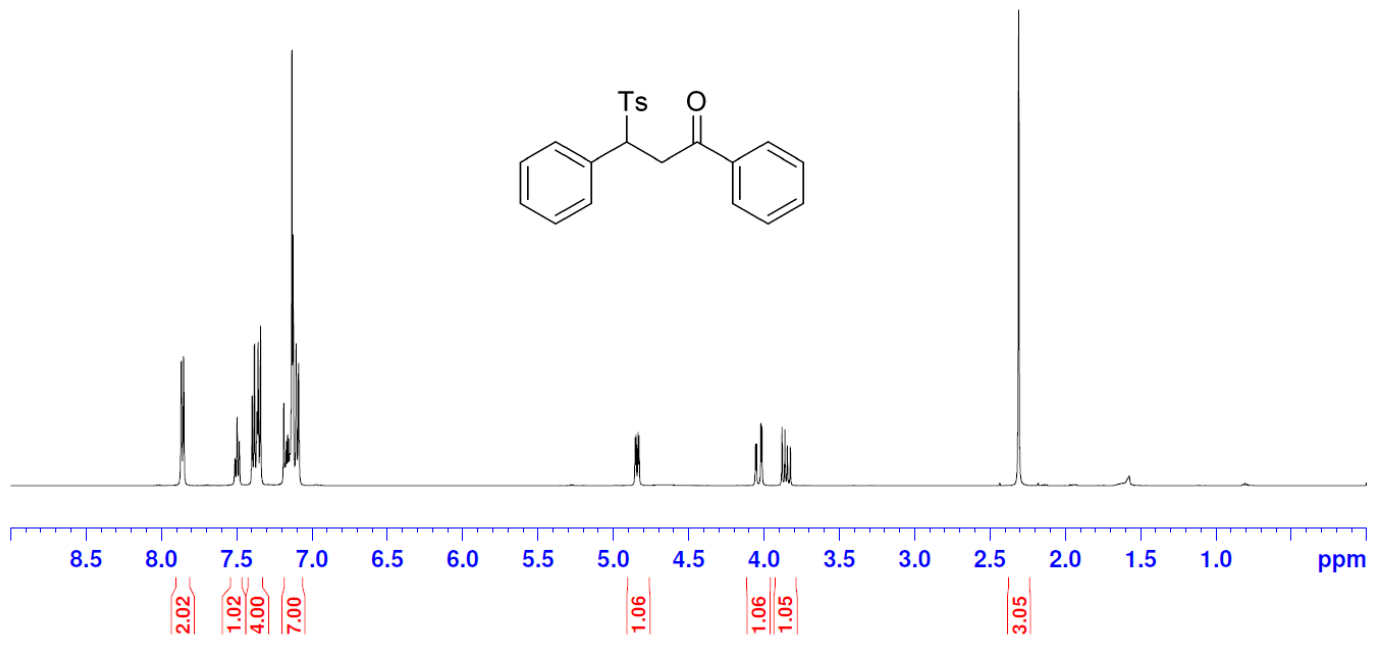

${ }^{1} \mathrm{H}$ NMR of $3 \mathrm{a}$
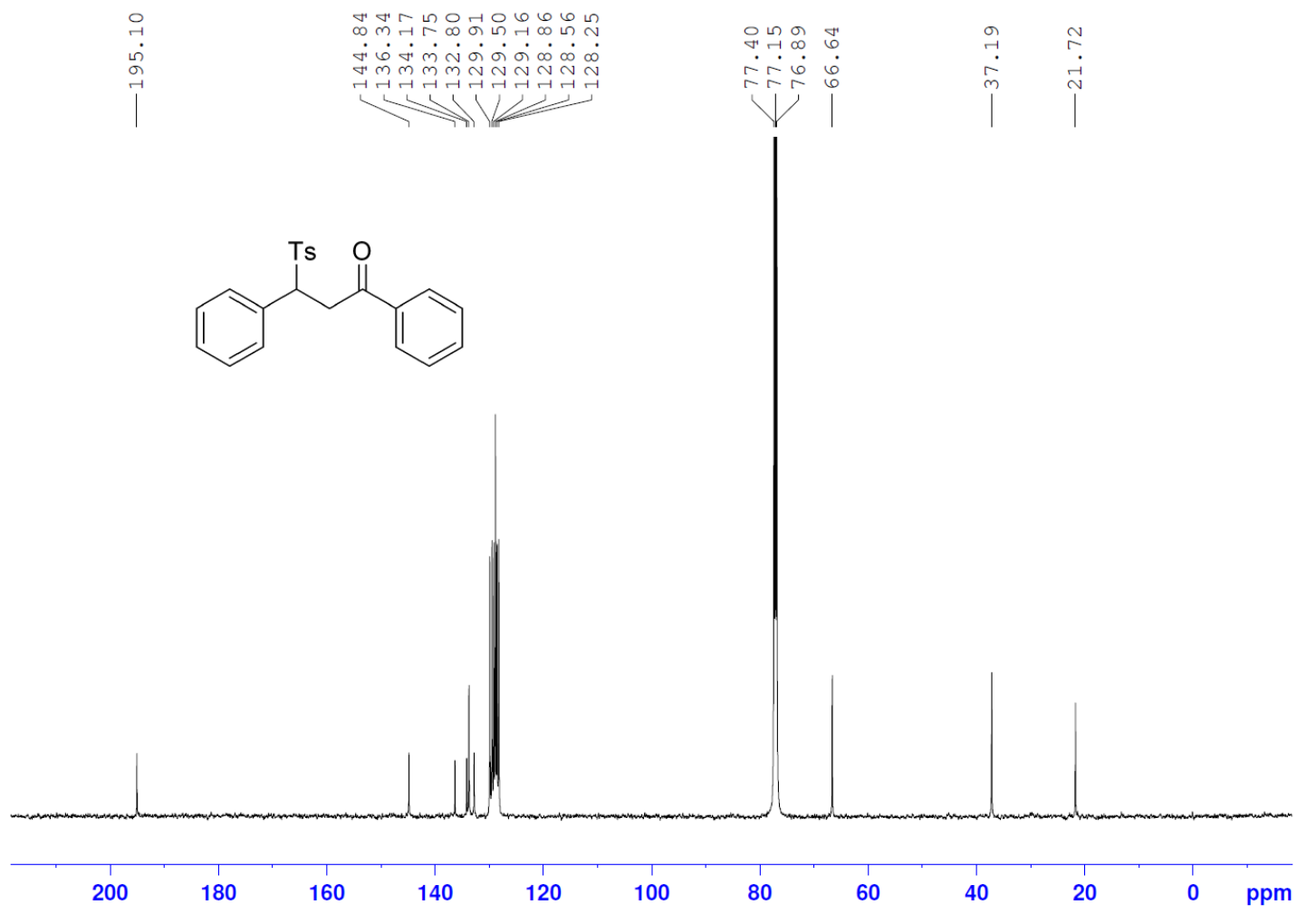

${ }^{13}$ C NMR of Ba

S14 


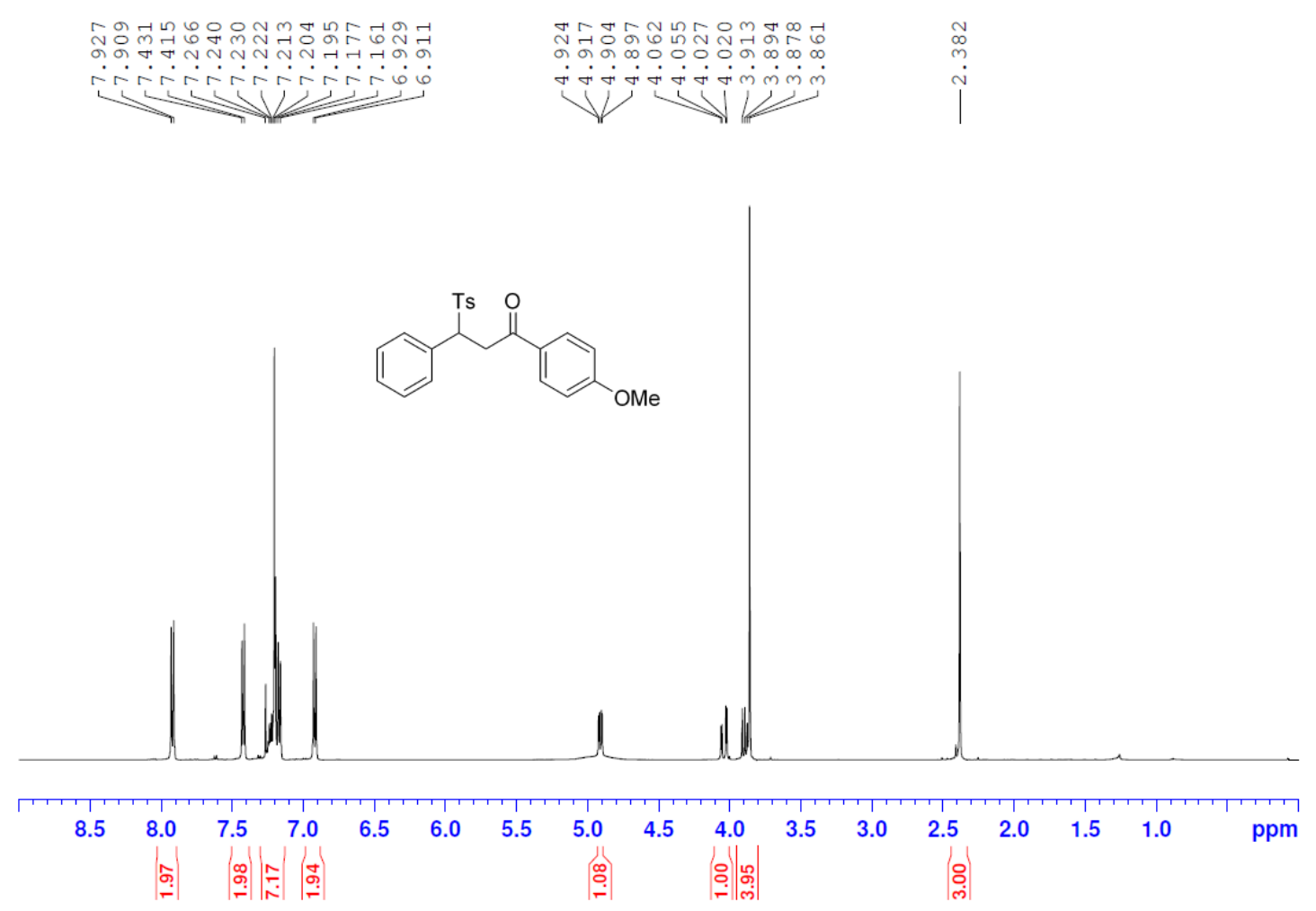

${ }^{1} \mathbf{H}$ NMR of $\mathbf{3 b}$

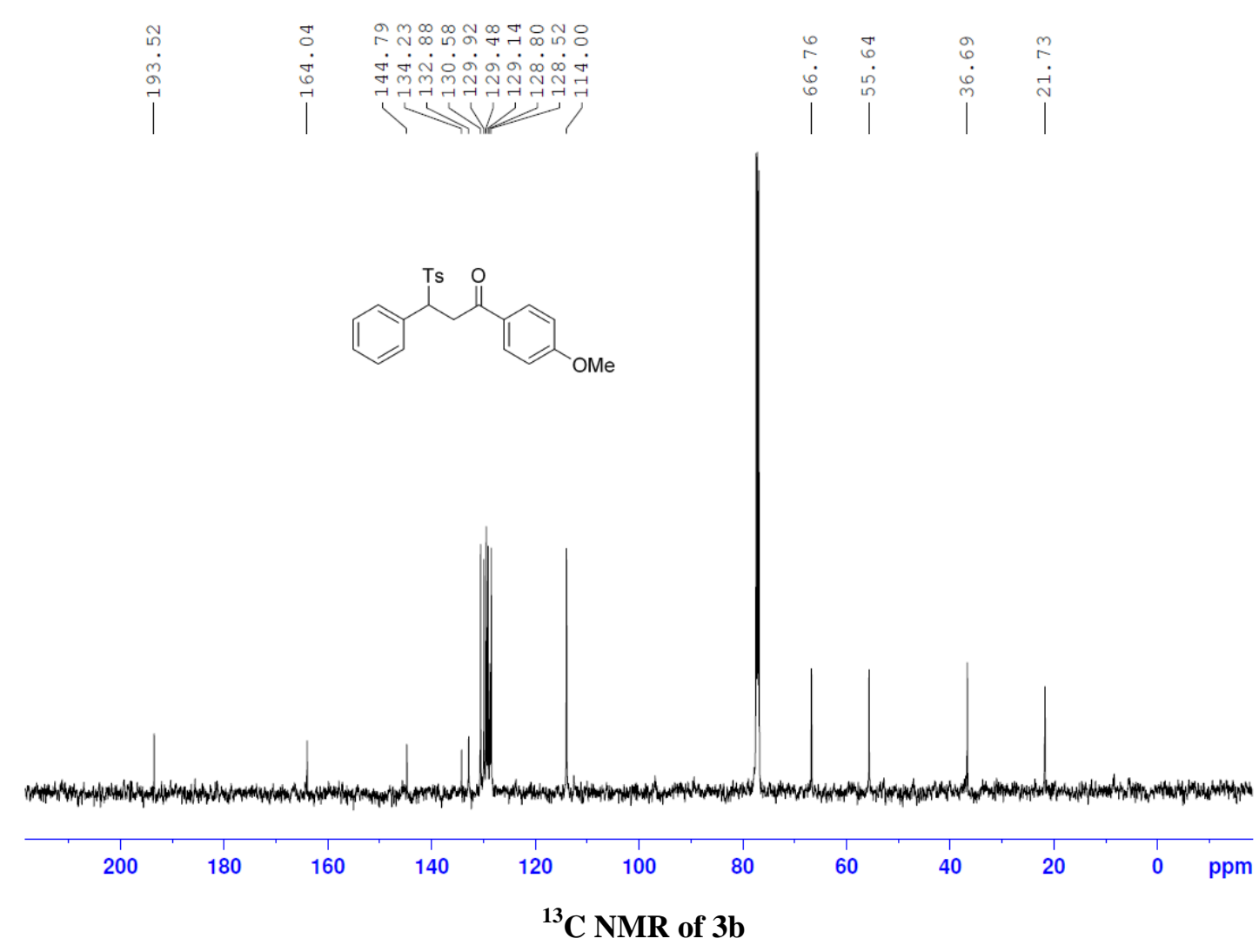



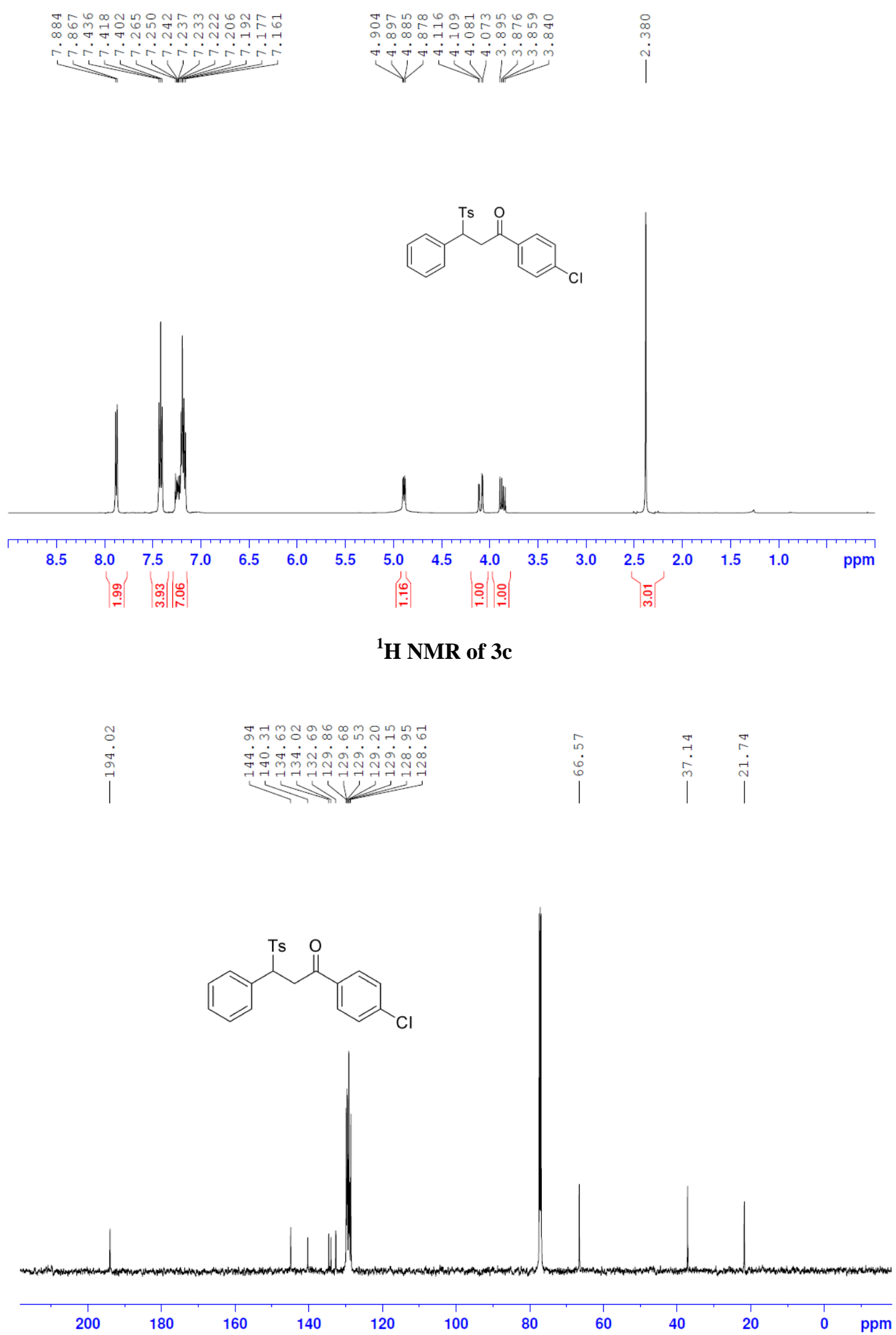

${ }^{13}$ C NMR of $3 \mathrm{c}$ 


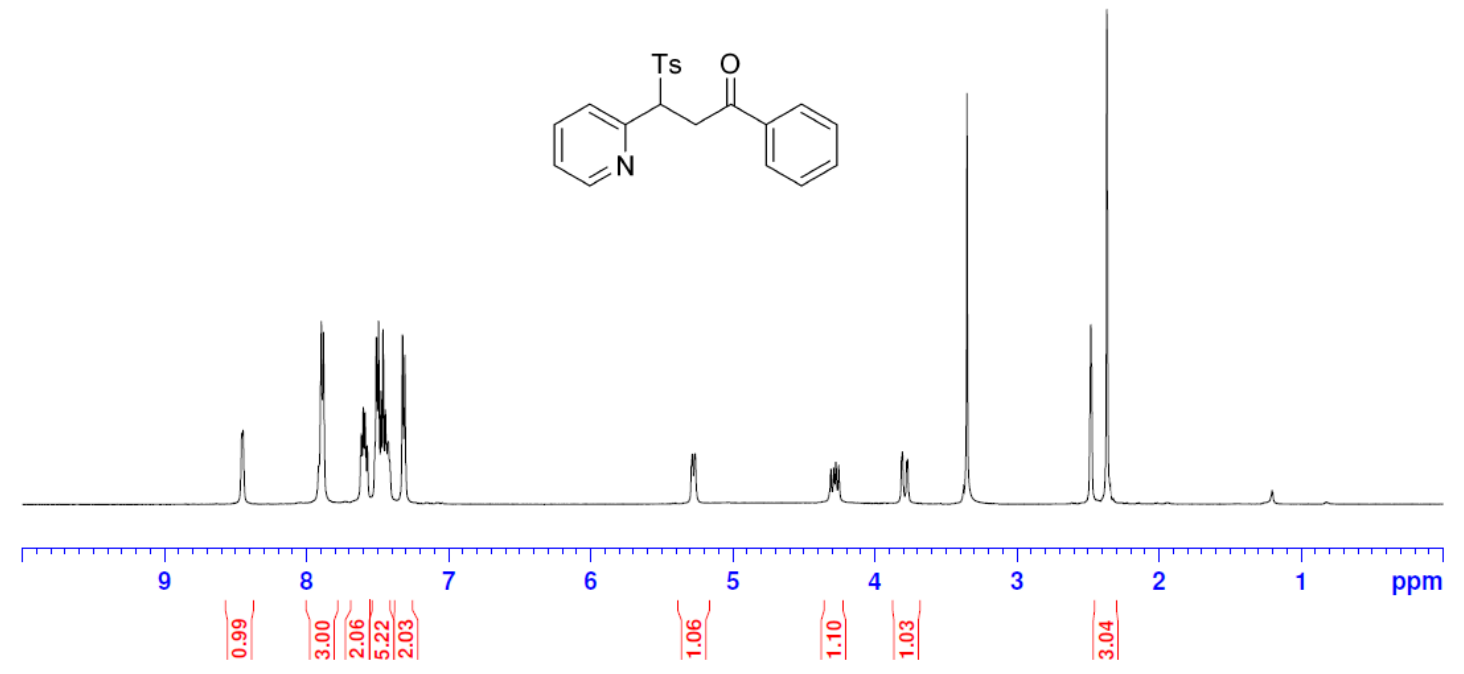

${ }^{1} \mathrm{H}$ NMR of $\mathbf{3 d}$

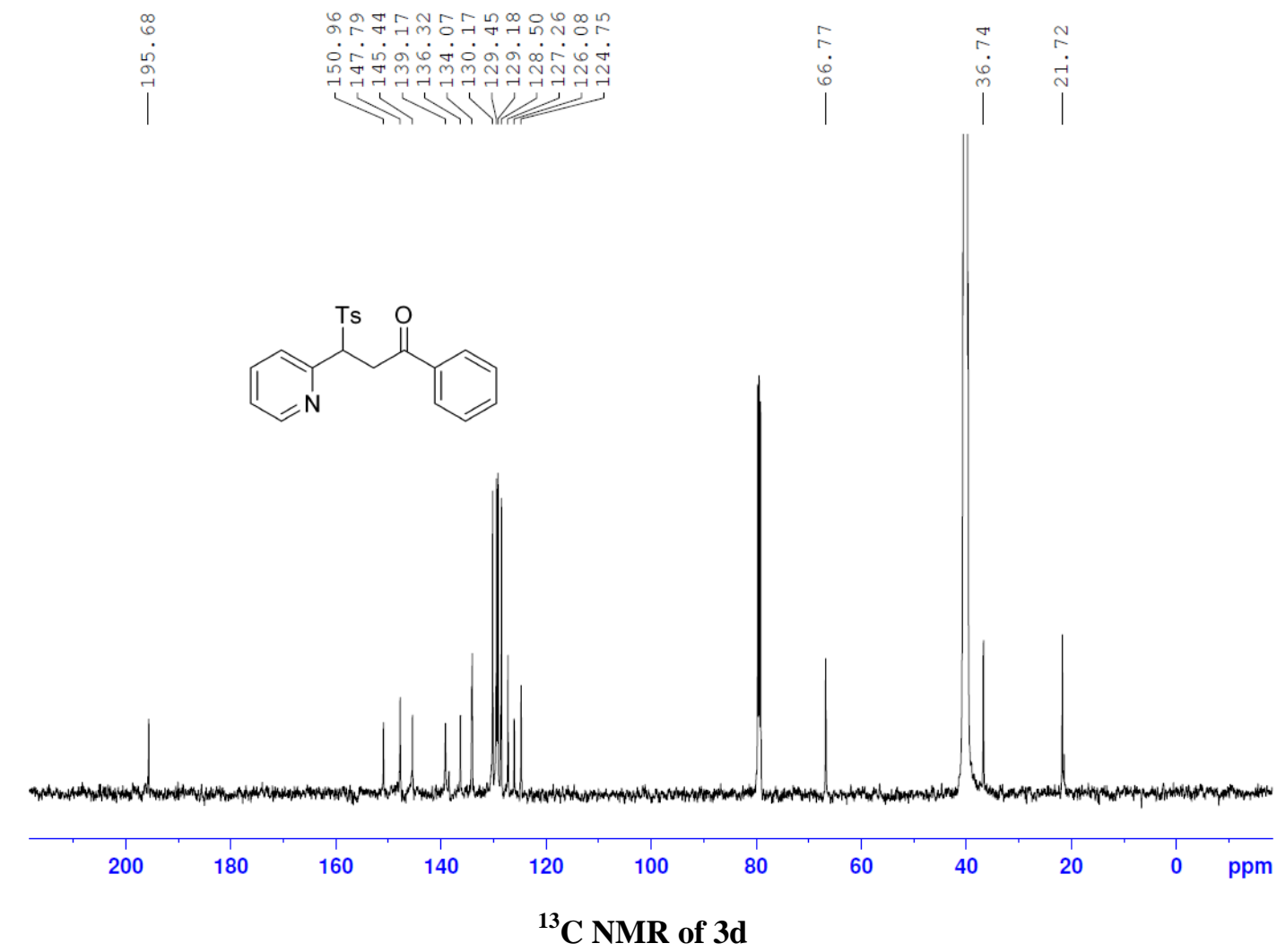



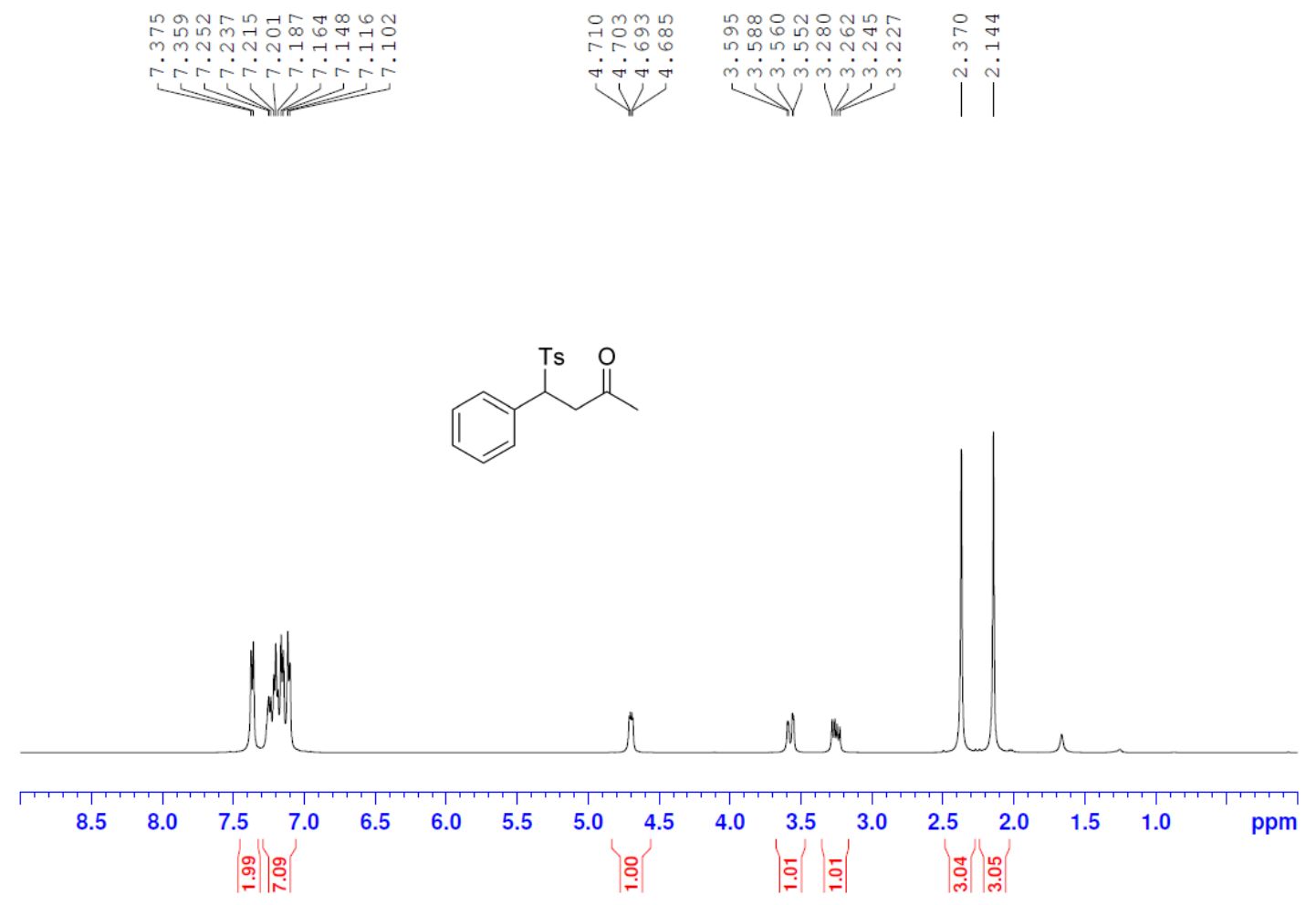

${ }^{1} \mathrm{H}$ NMR of $3 \mathrm{e}$

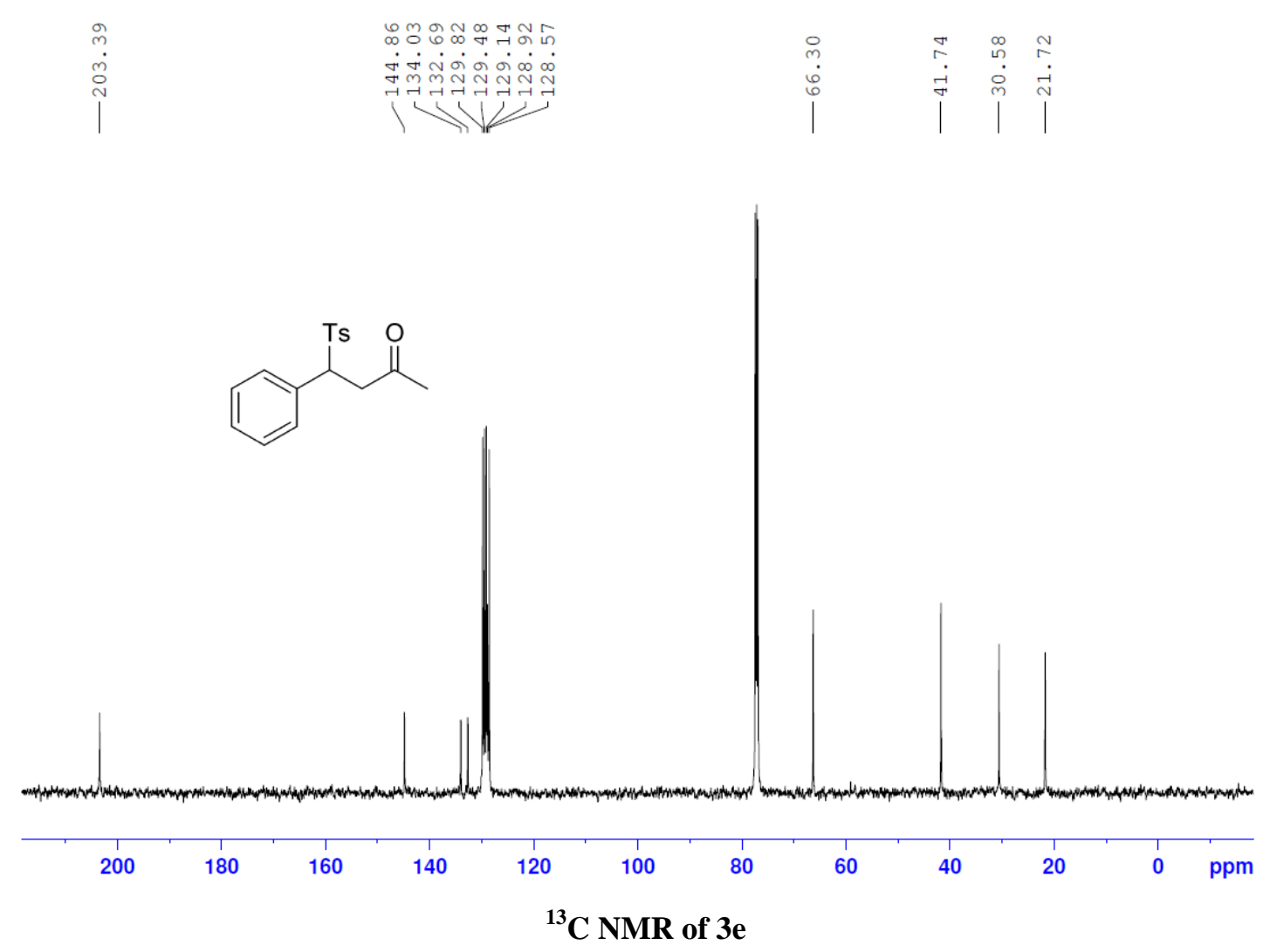



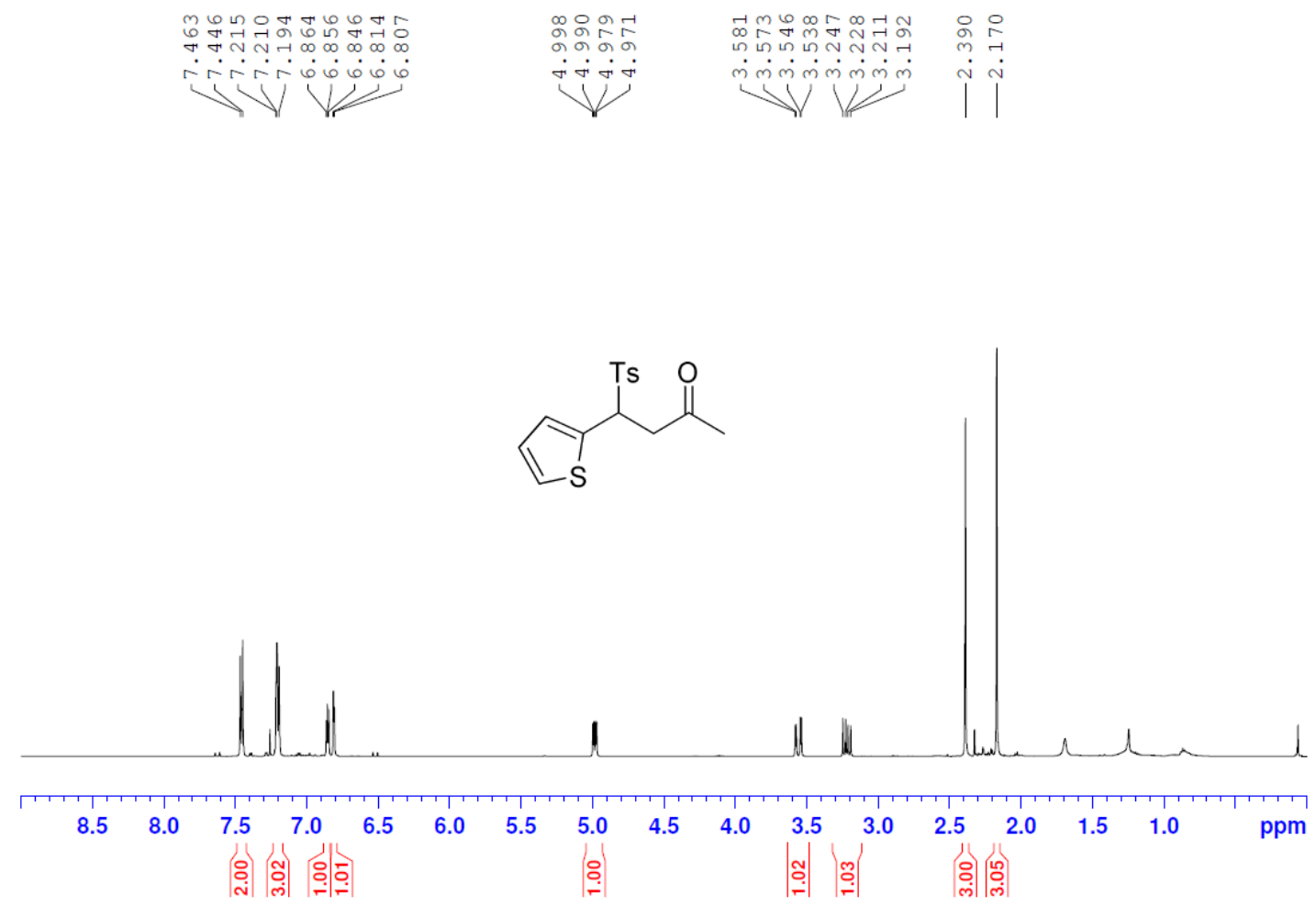

${ }^{1}$ H NMR of $3 f$

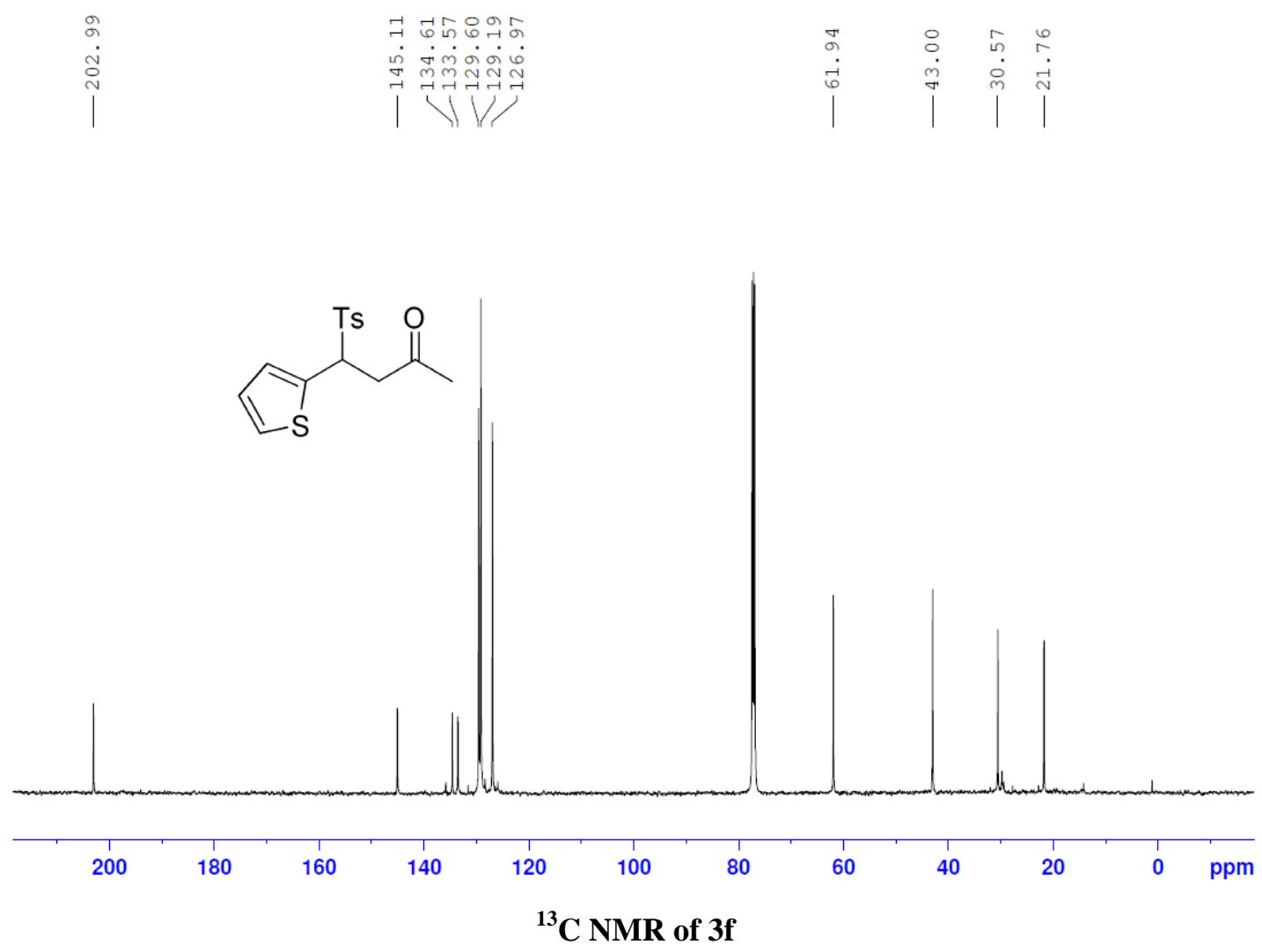




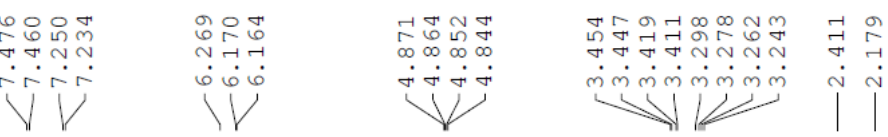<smiles>CC(=O)CC([Te])c1ccco1</smiles>

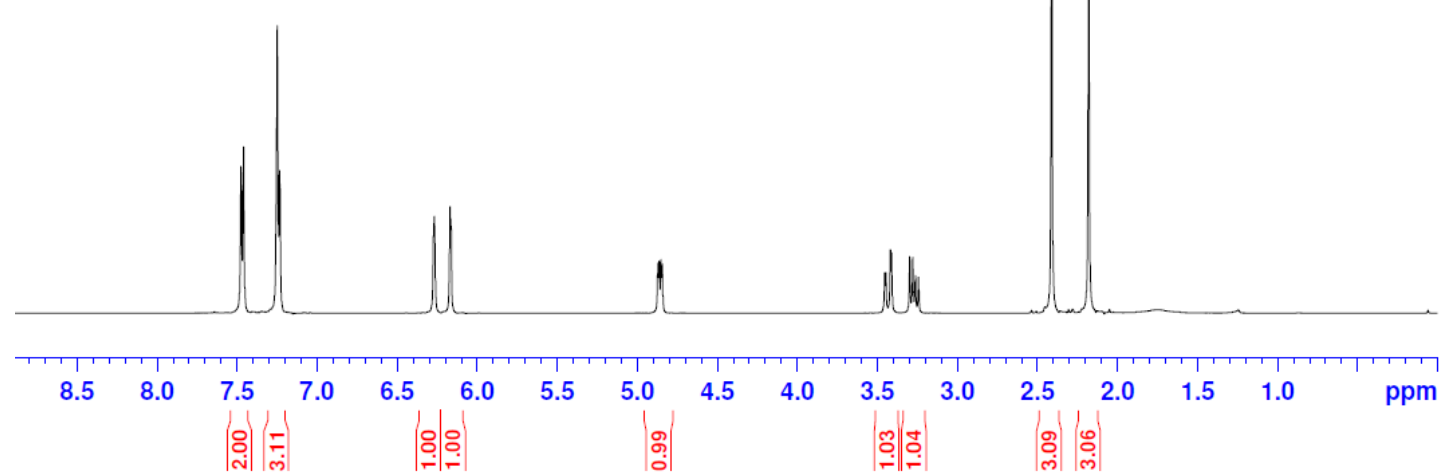

${ }^{1} \mathrm{H}$ NMR of $\mathbf{3 g}$

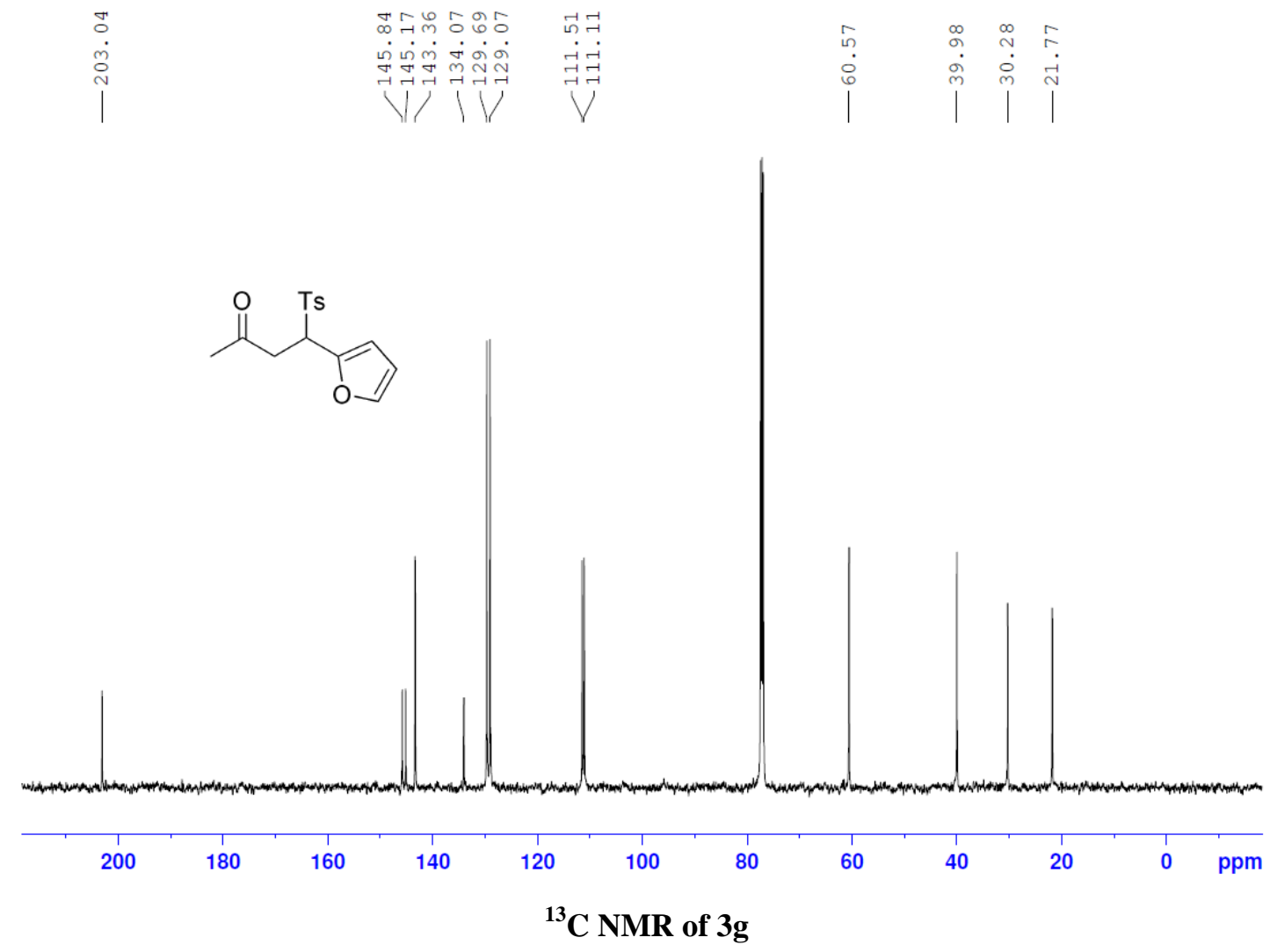




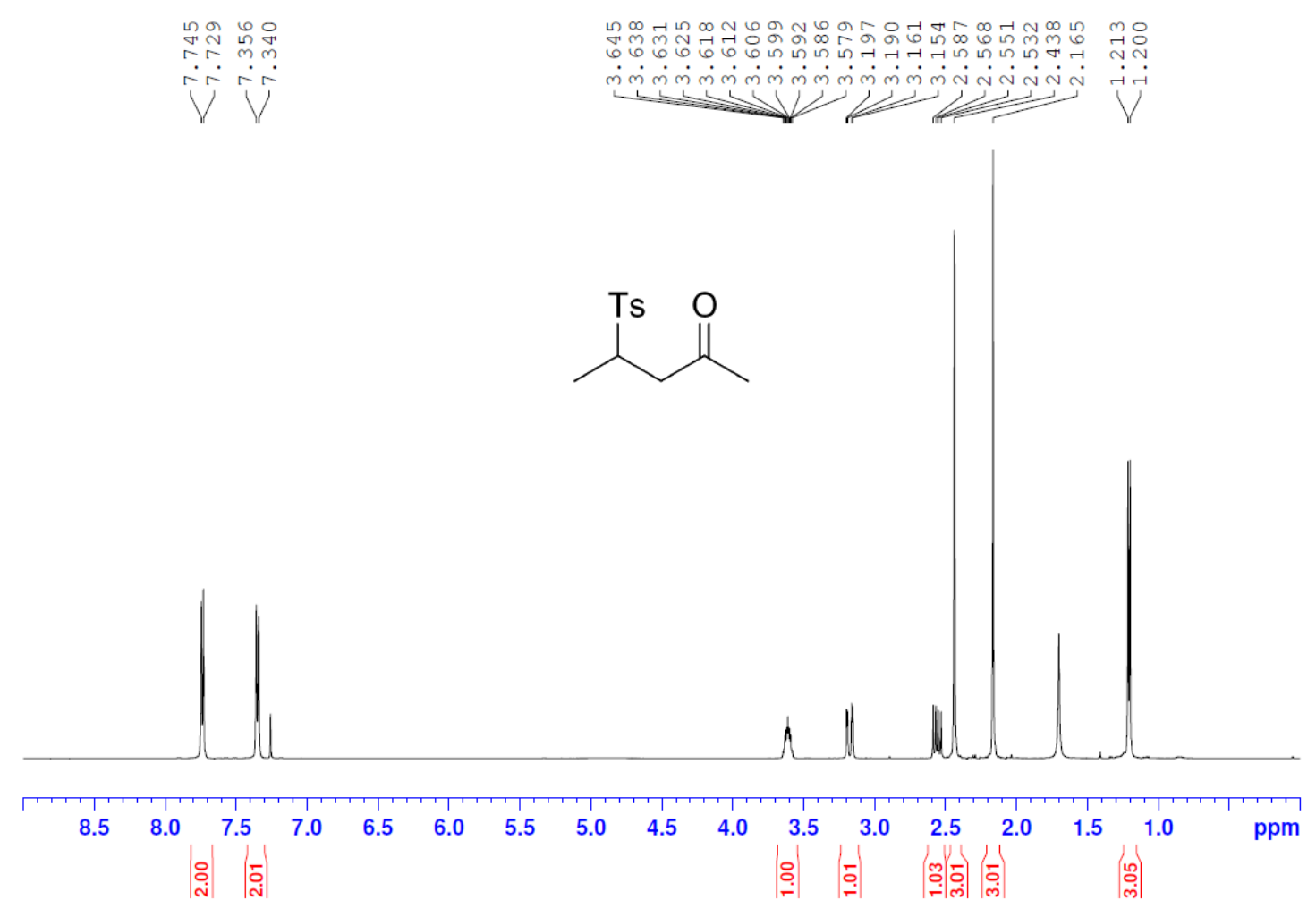

${ }^{\mathbf{1}} \mathrm{H}$ NMR of $\mathbf{3 h}$

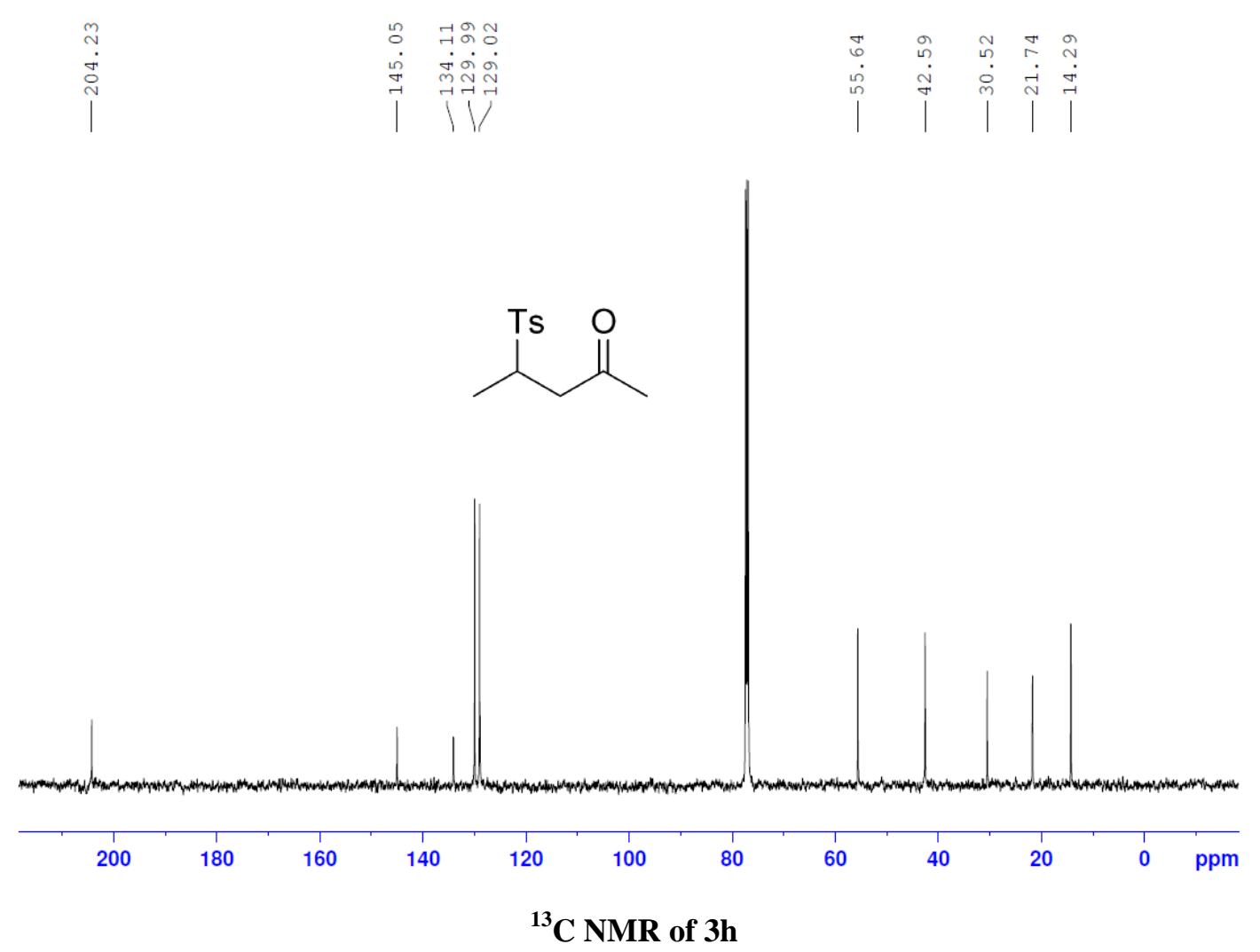




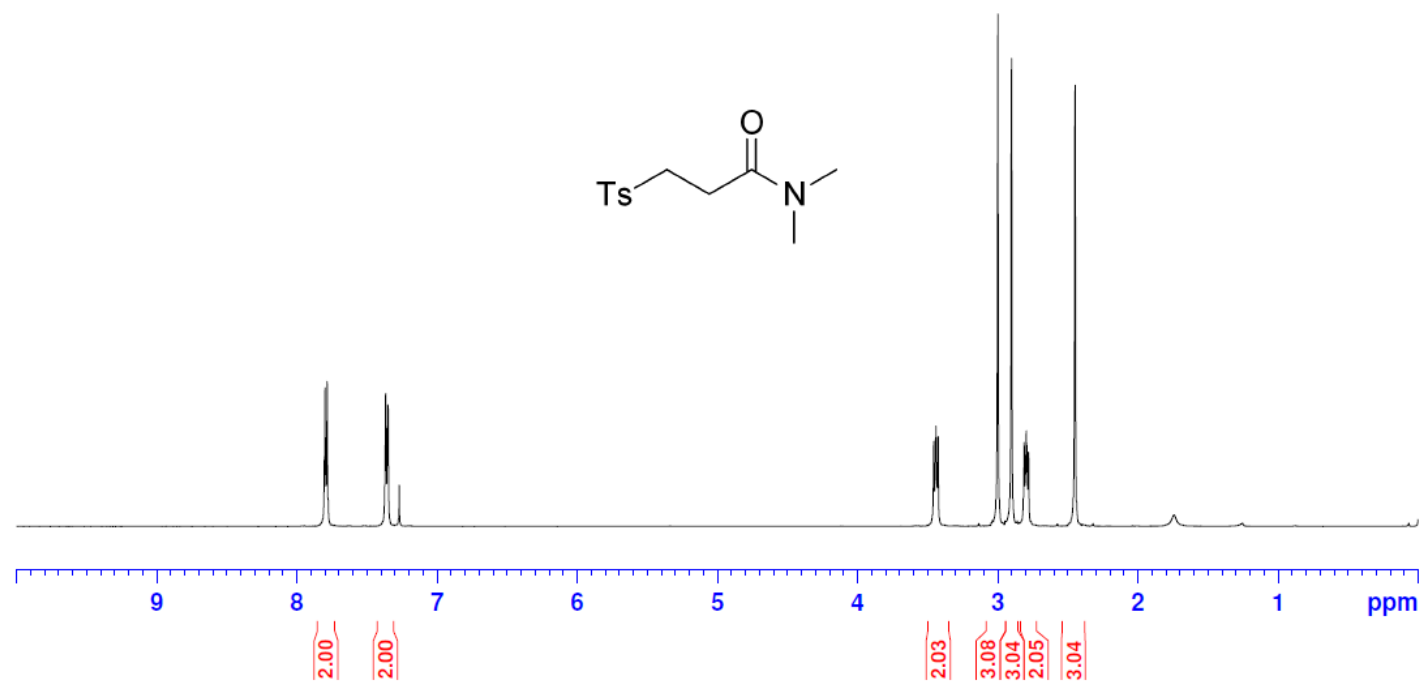

${ }^{1} \mathrm{H}$ NMR of $3 \mathrm{i}$

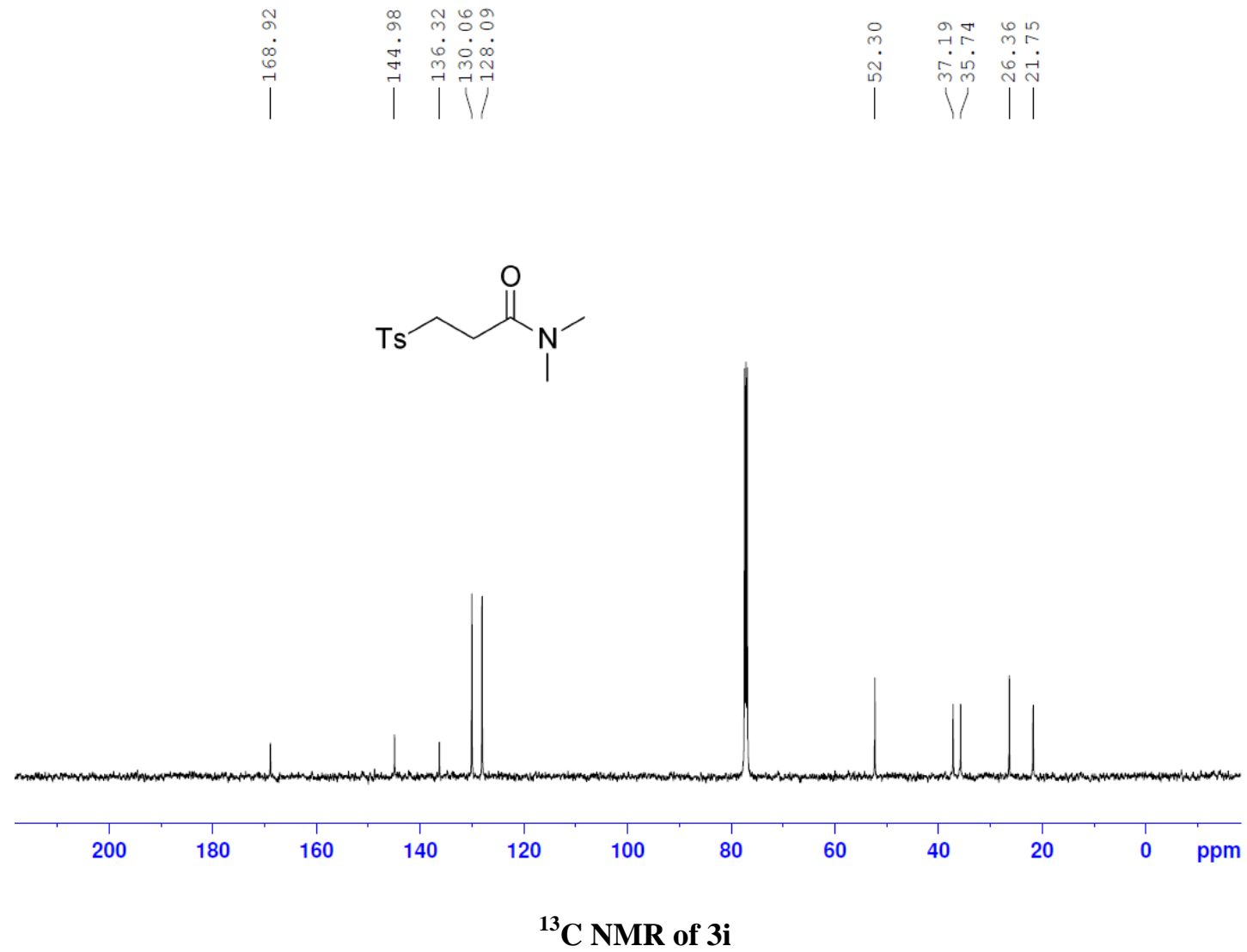



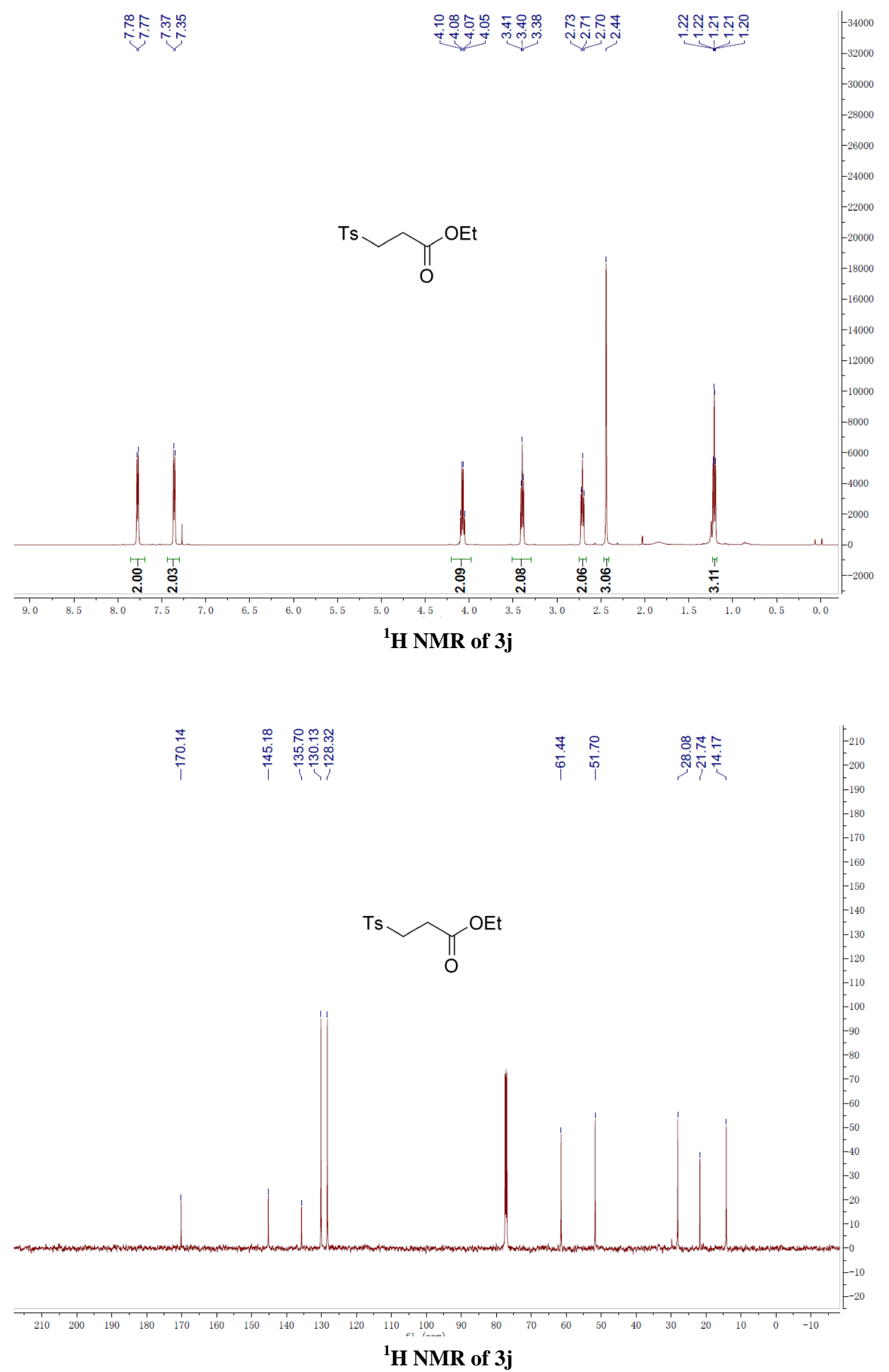


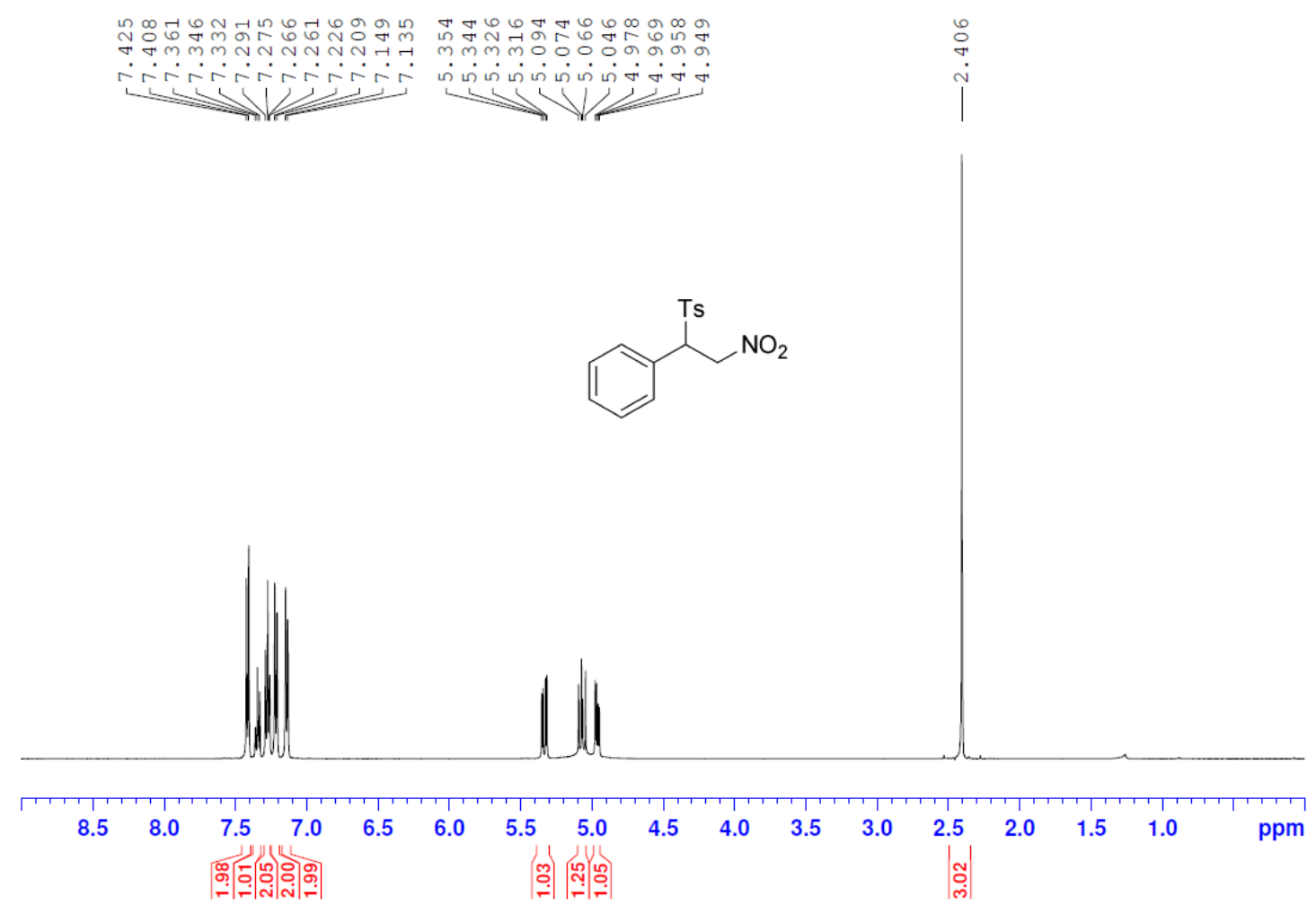

${ }^{1} \mathrm{H}$ NMR of $3 \mathrm{k}$
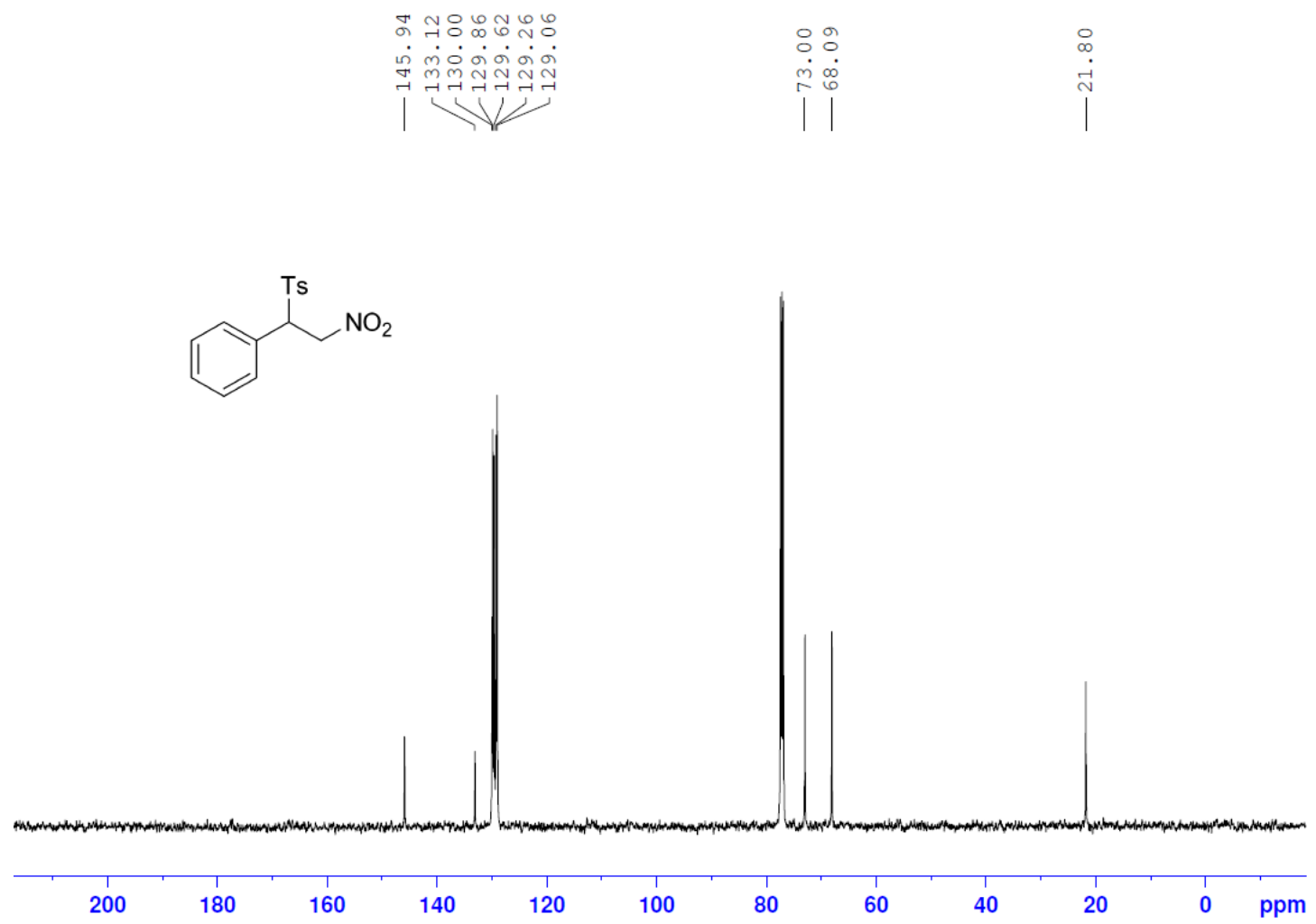

${ }^{13} \mathrm{C}$ NMR of $3 k$ 


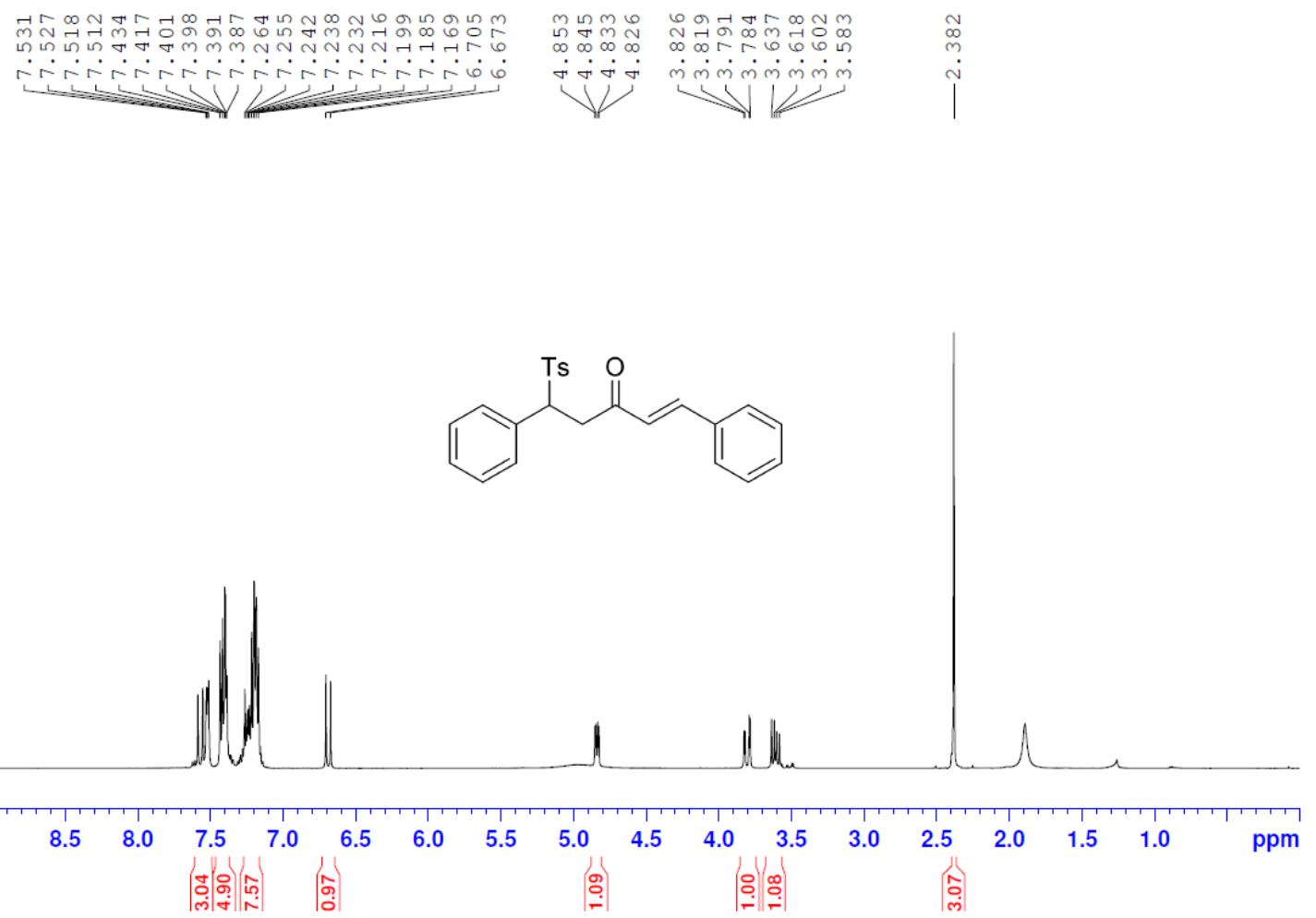

${ }^{1} \mathrm{H}$ NMR of 3 l

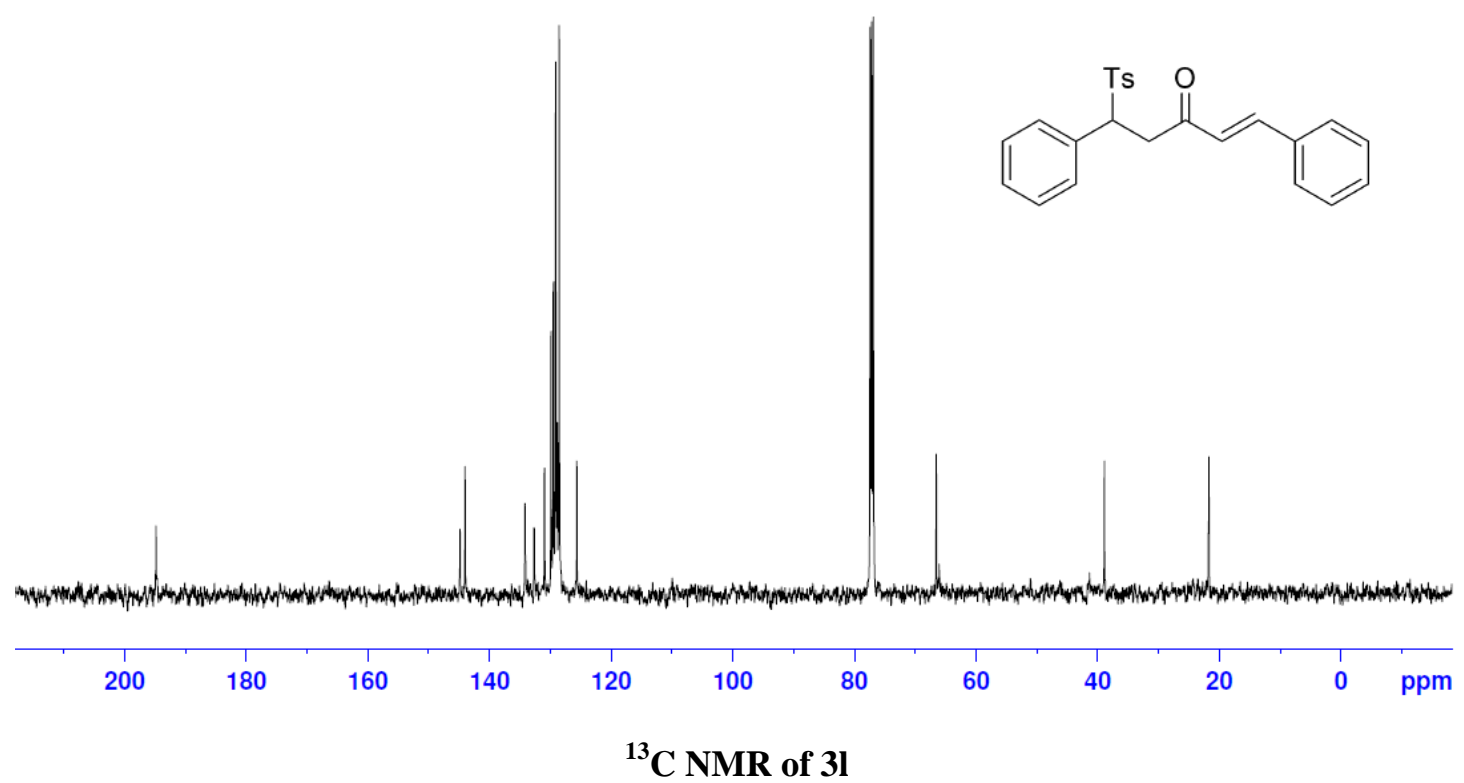




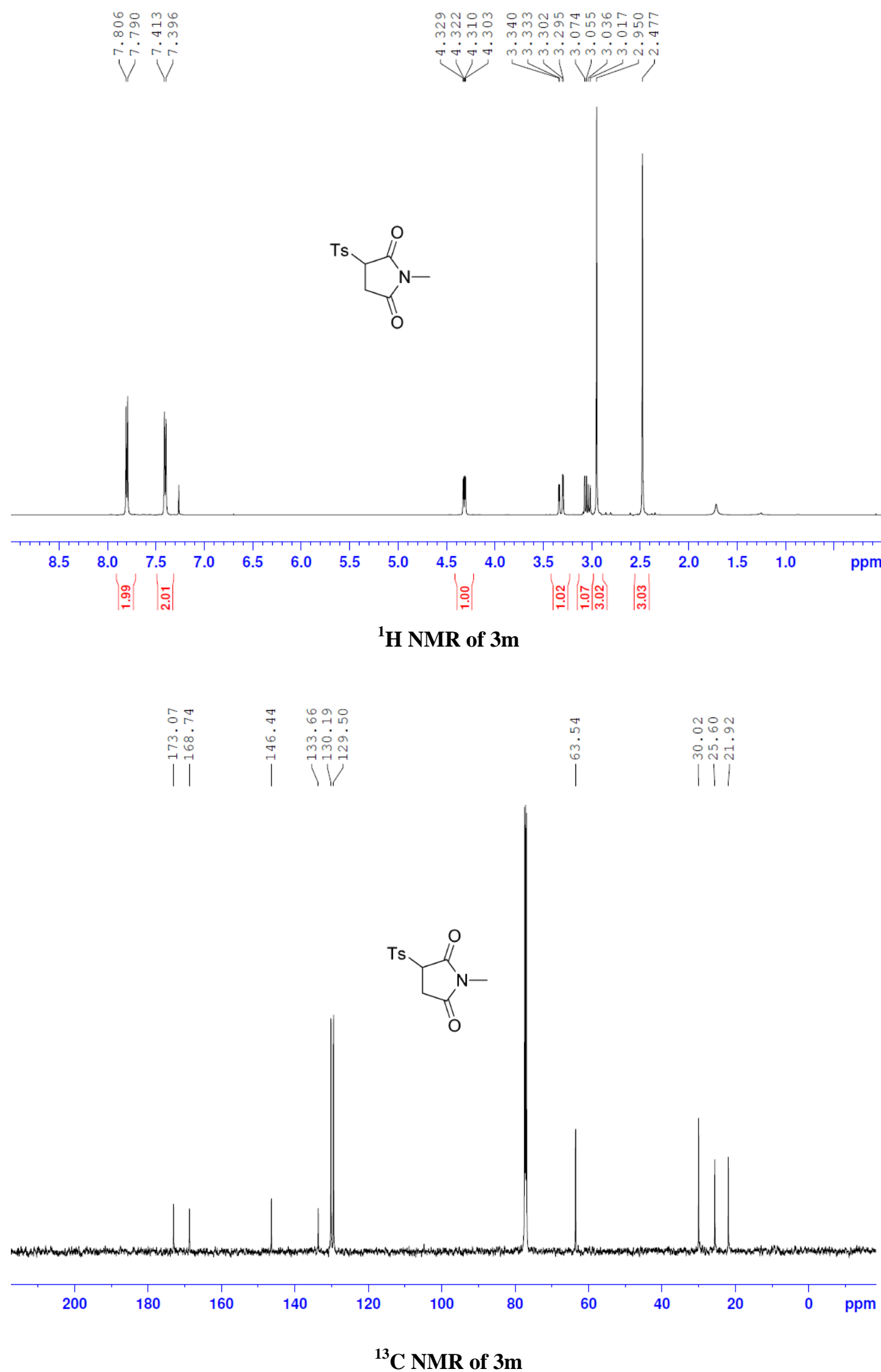




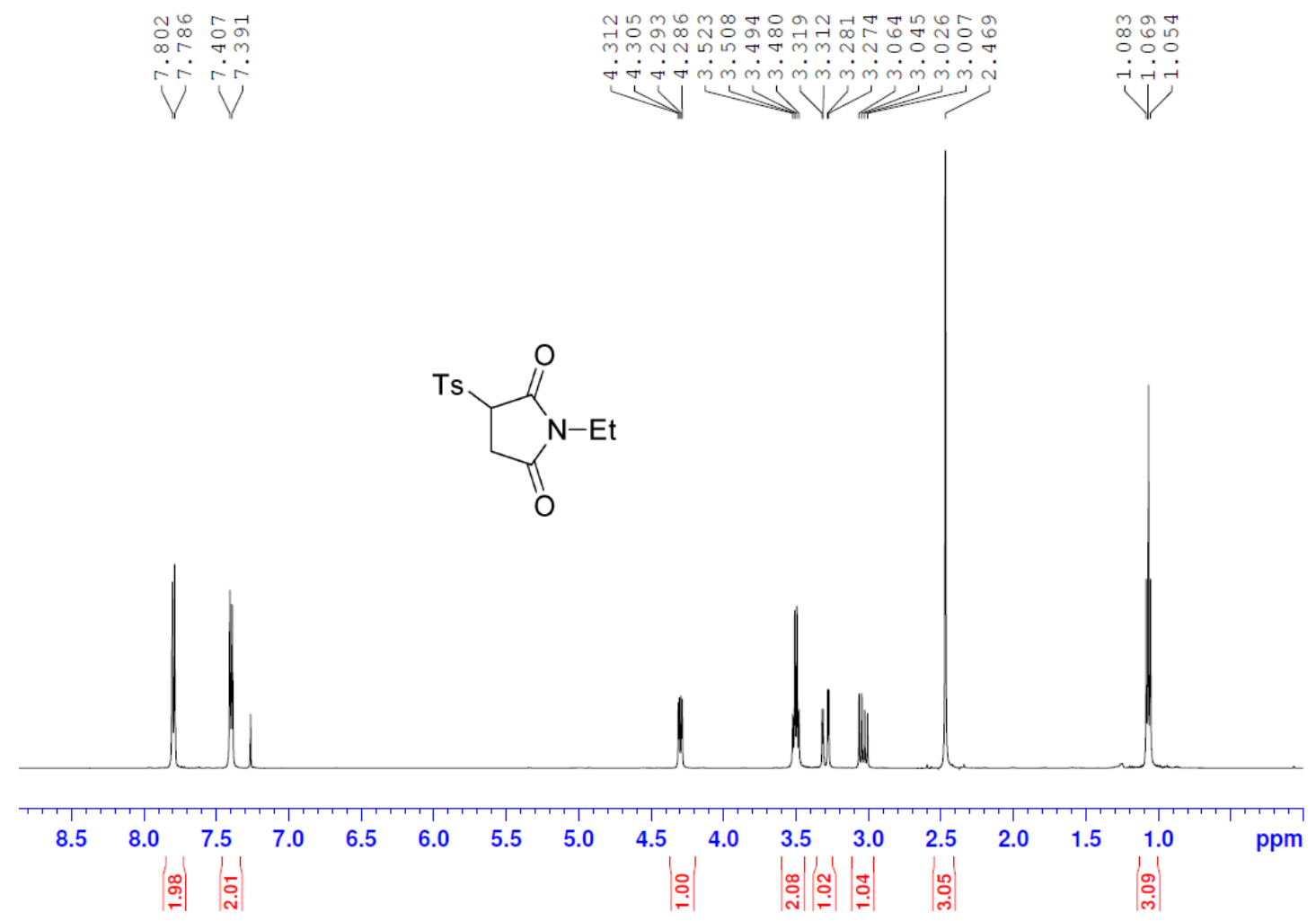

\section{${ }^{1}$ H NMR of 3n}

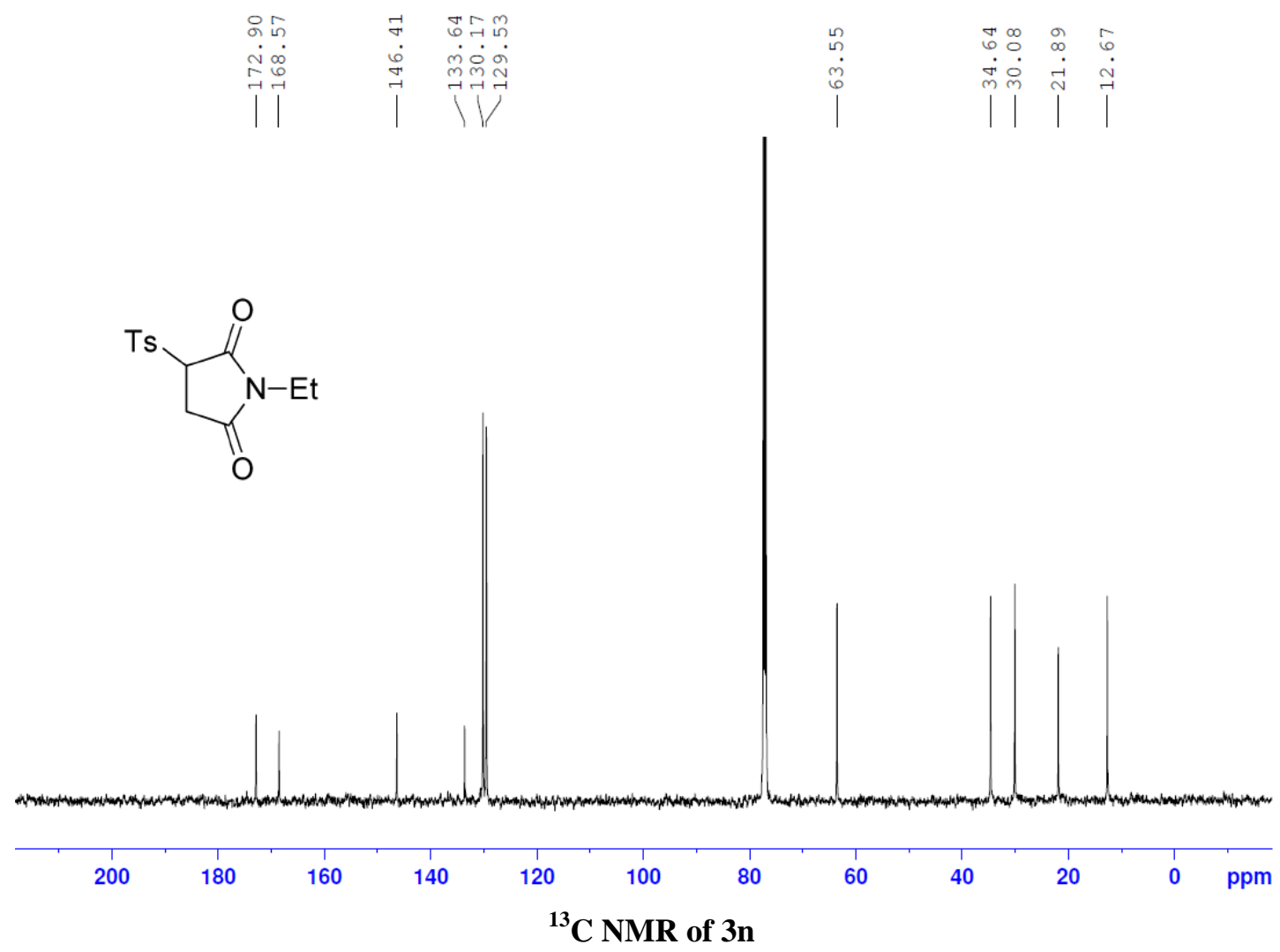




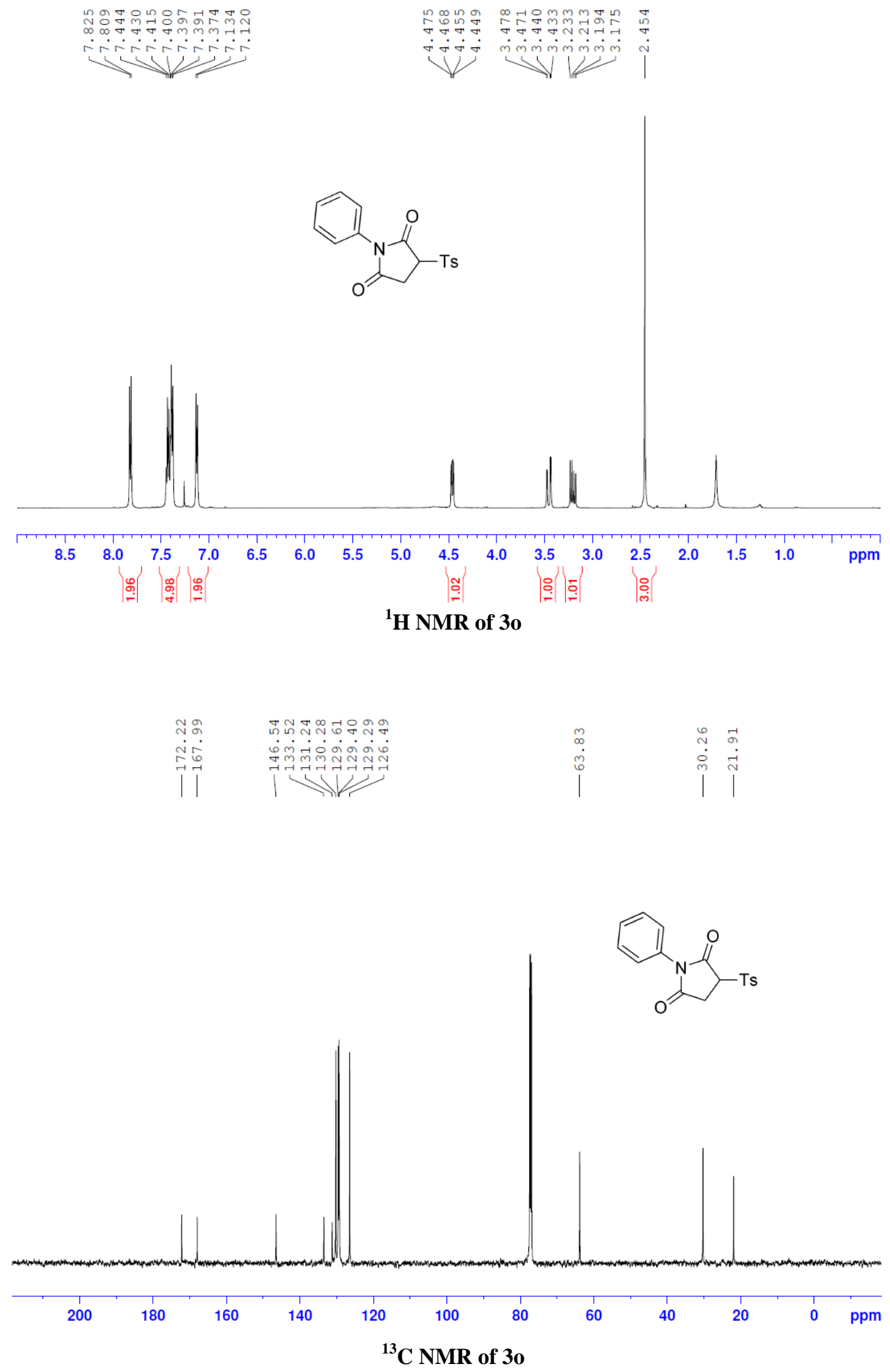




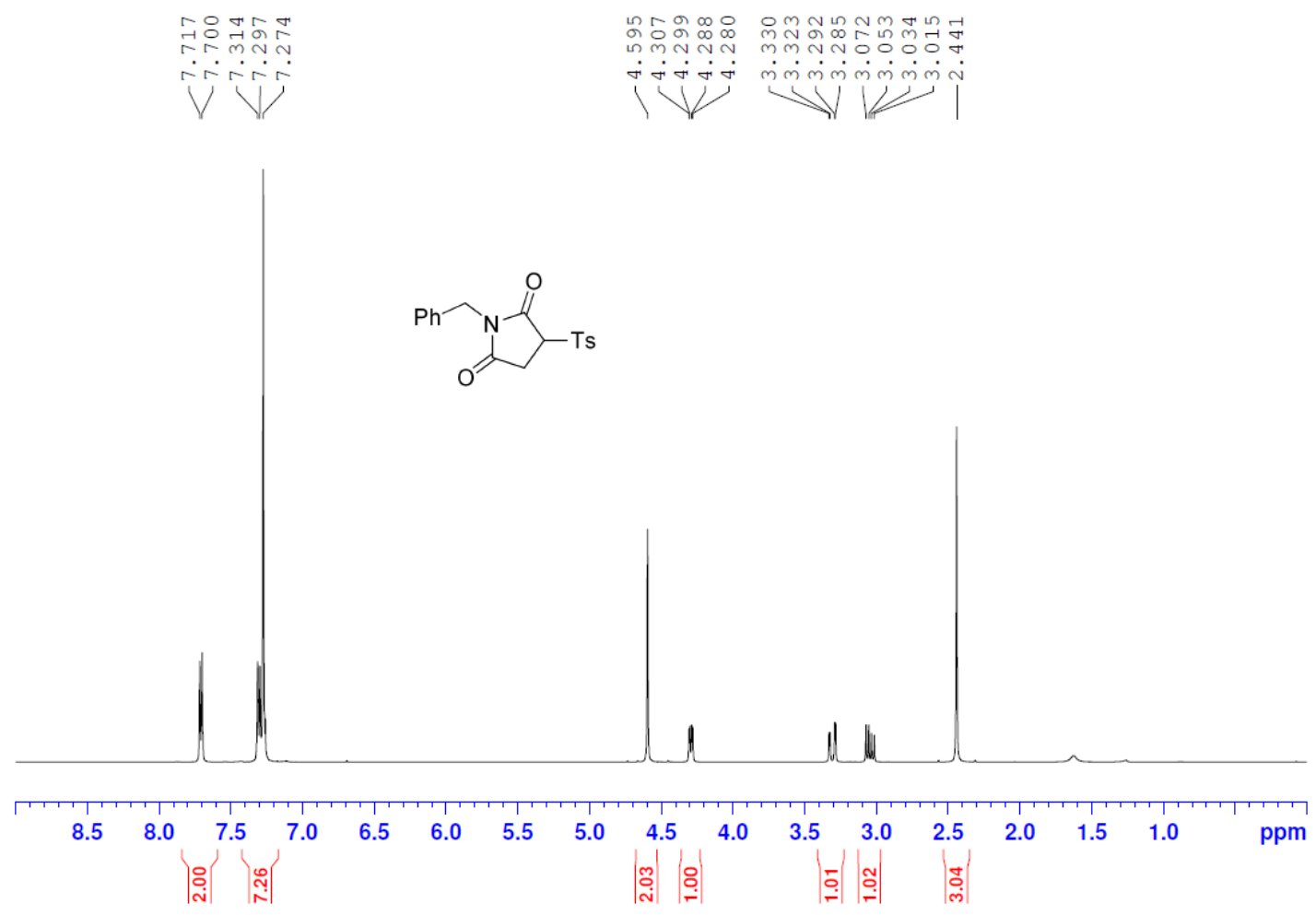

${ }^{1} \mathbf{H}$ NMR of $\mathbf{3 p}$

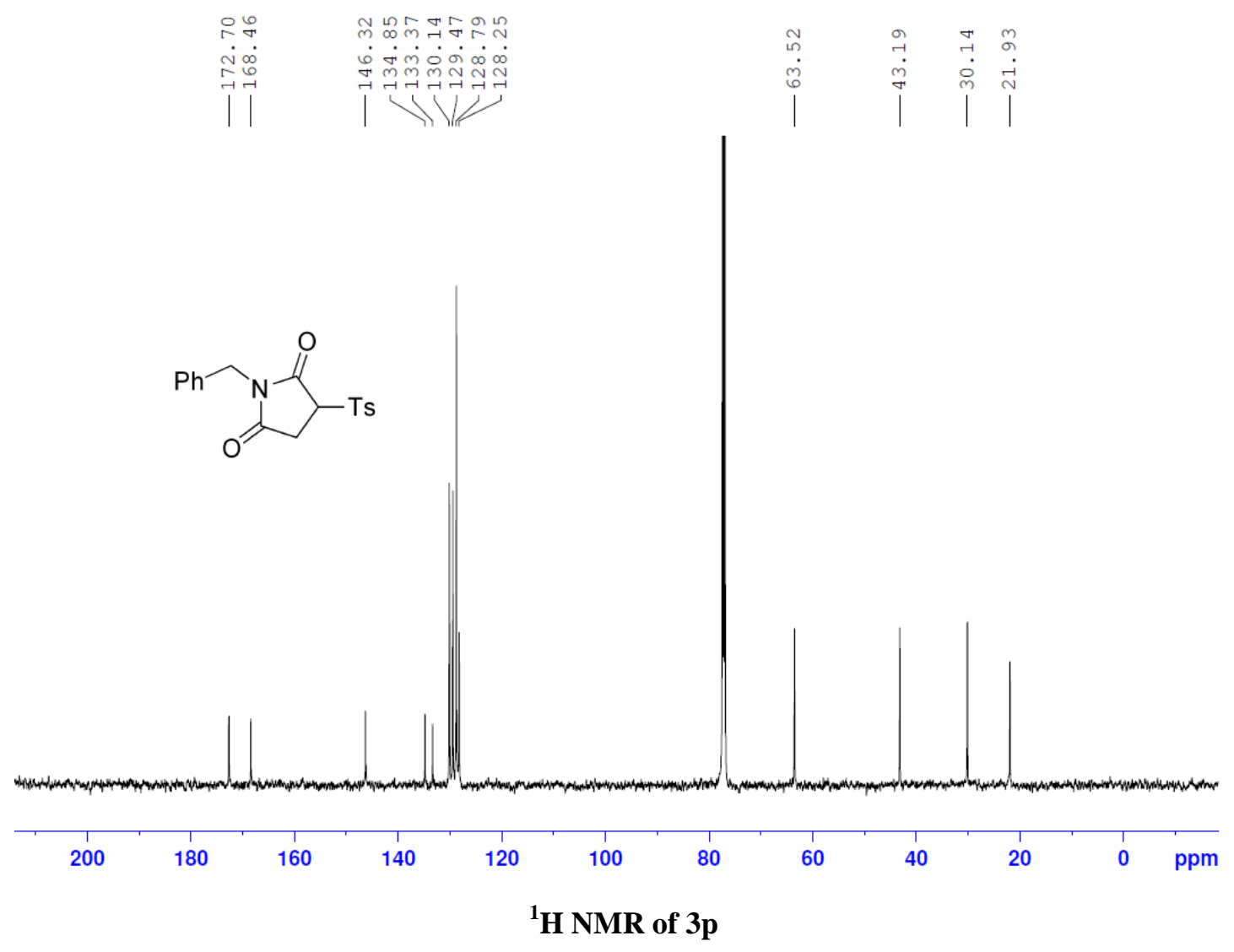



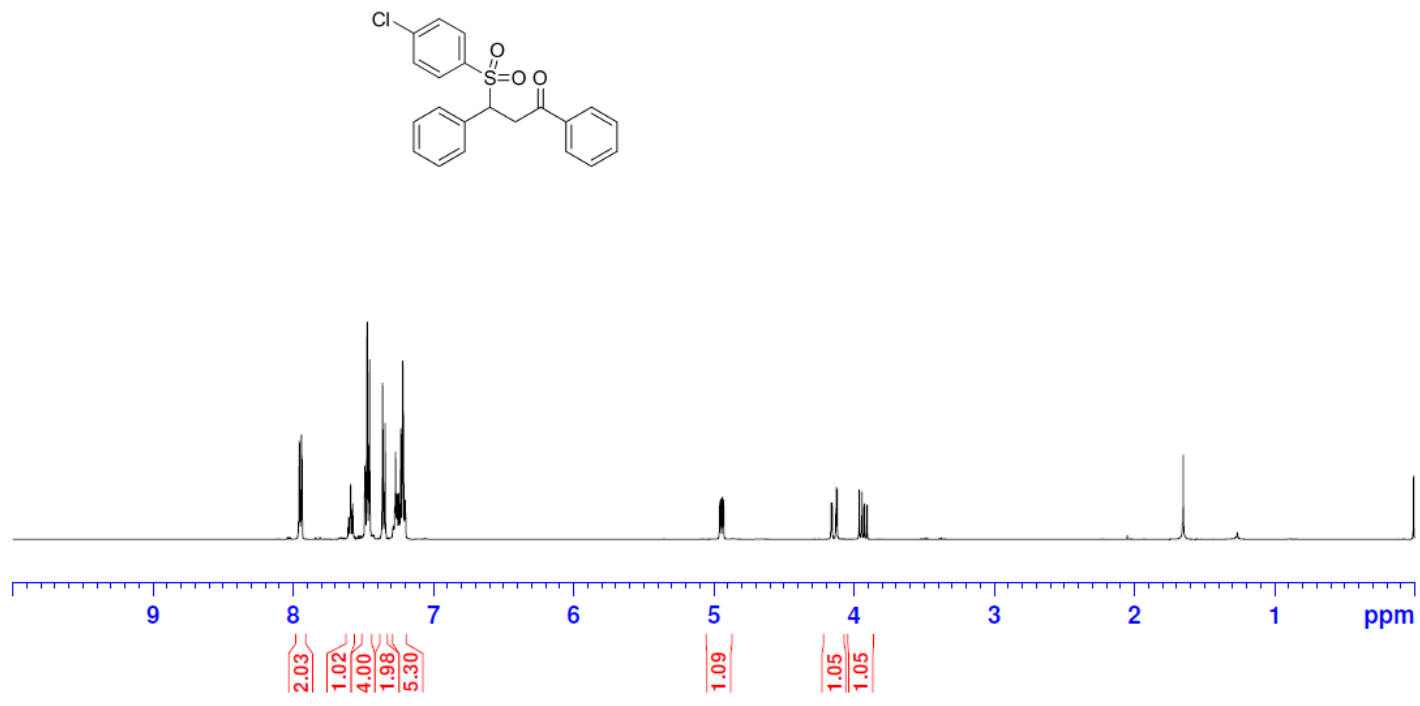

${ }^{1}$ H NMR of $3 q$

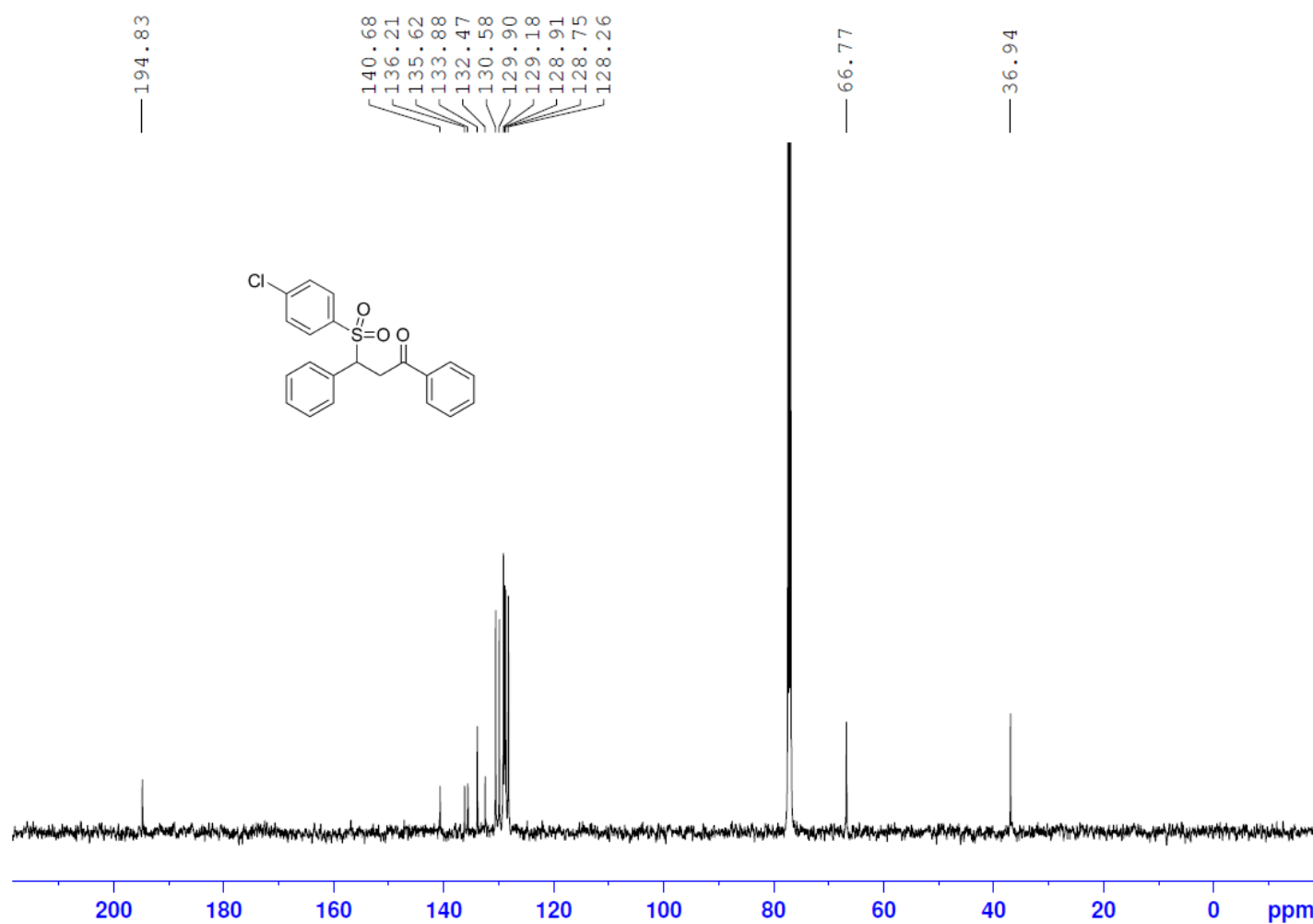

${ }^{13}$ C NMR of 3q 

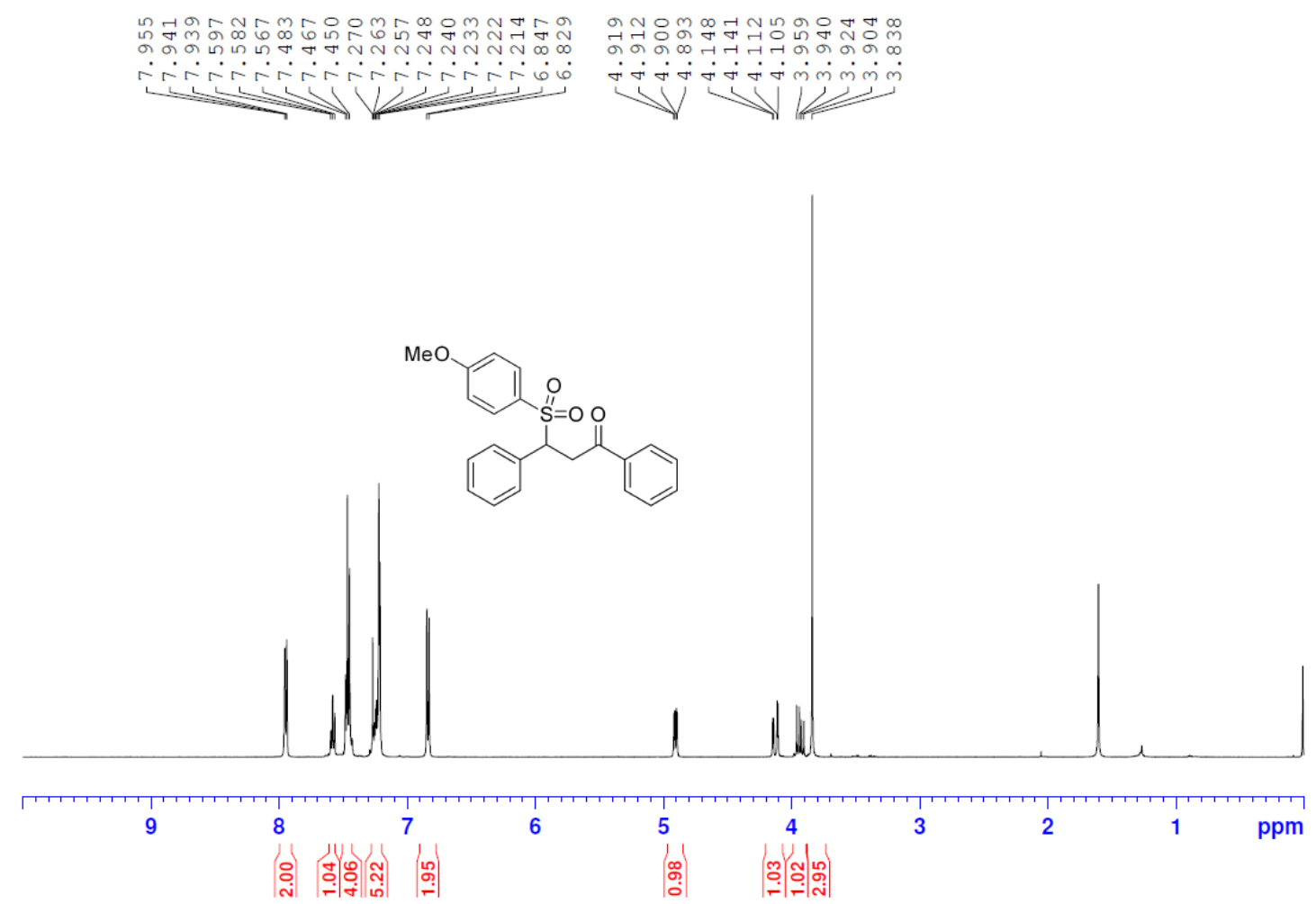

${ }^{1}$ H NMR of $3 \mathbf{r}$

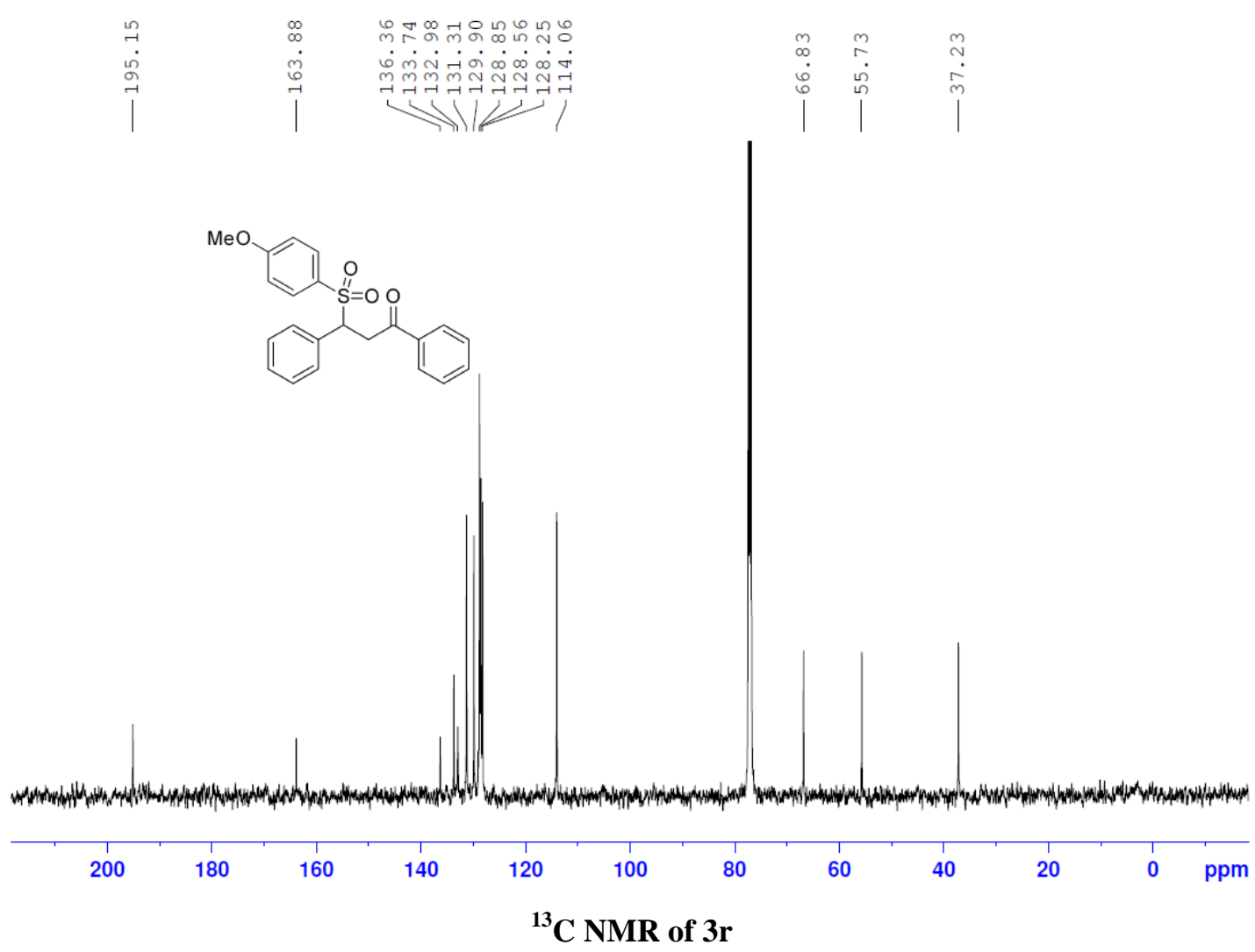




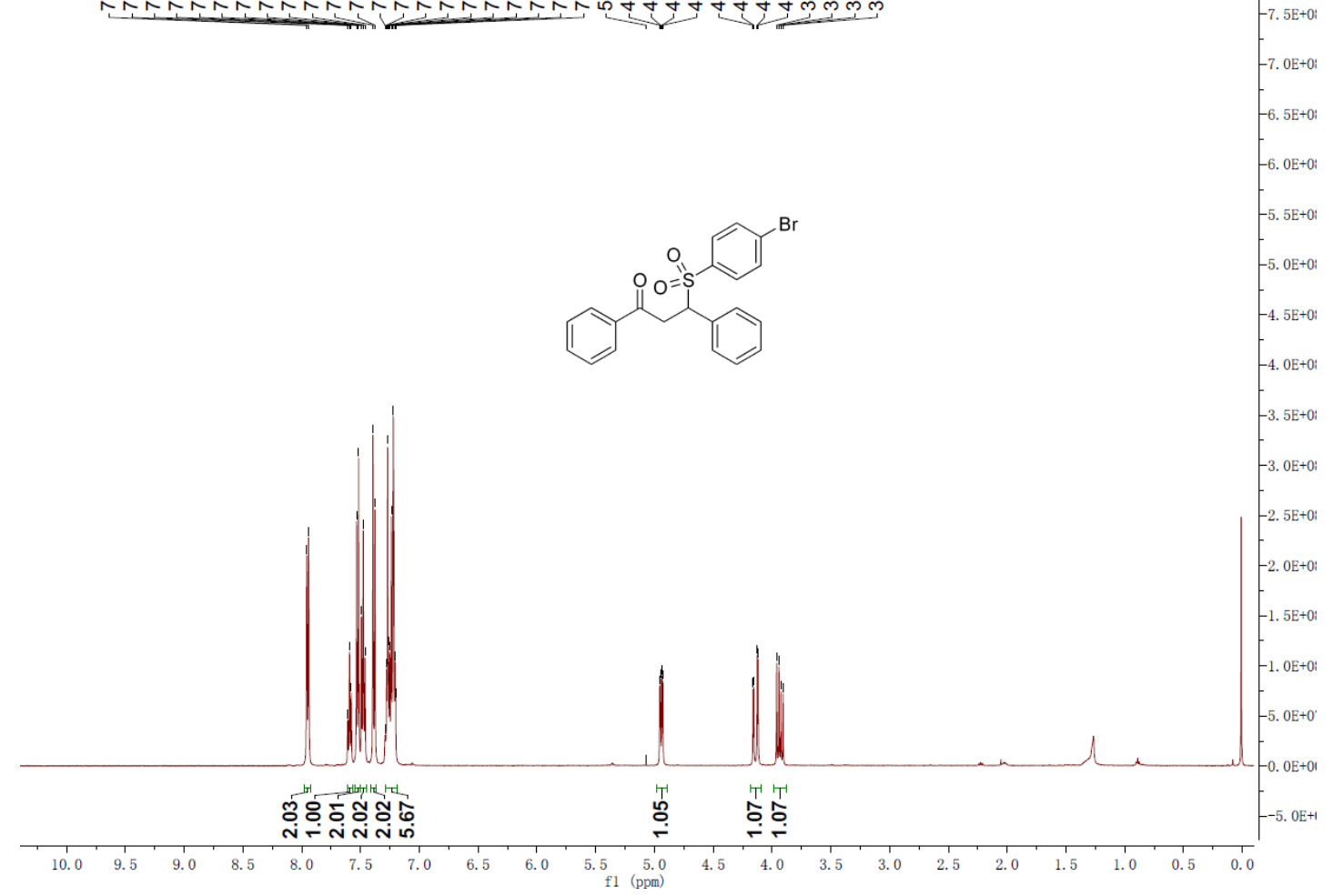

${ }^{1} \mathrm{H}$ NMR of $3 \mathrm{~s}$

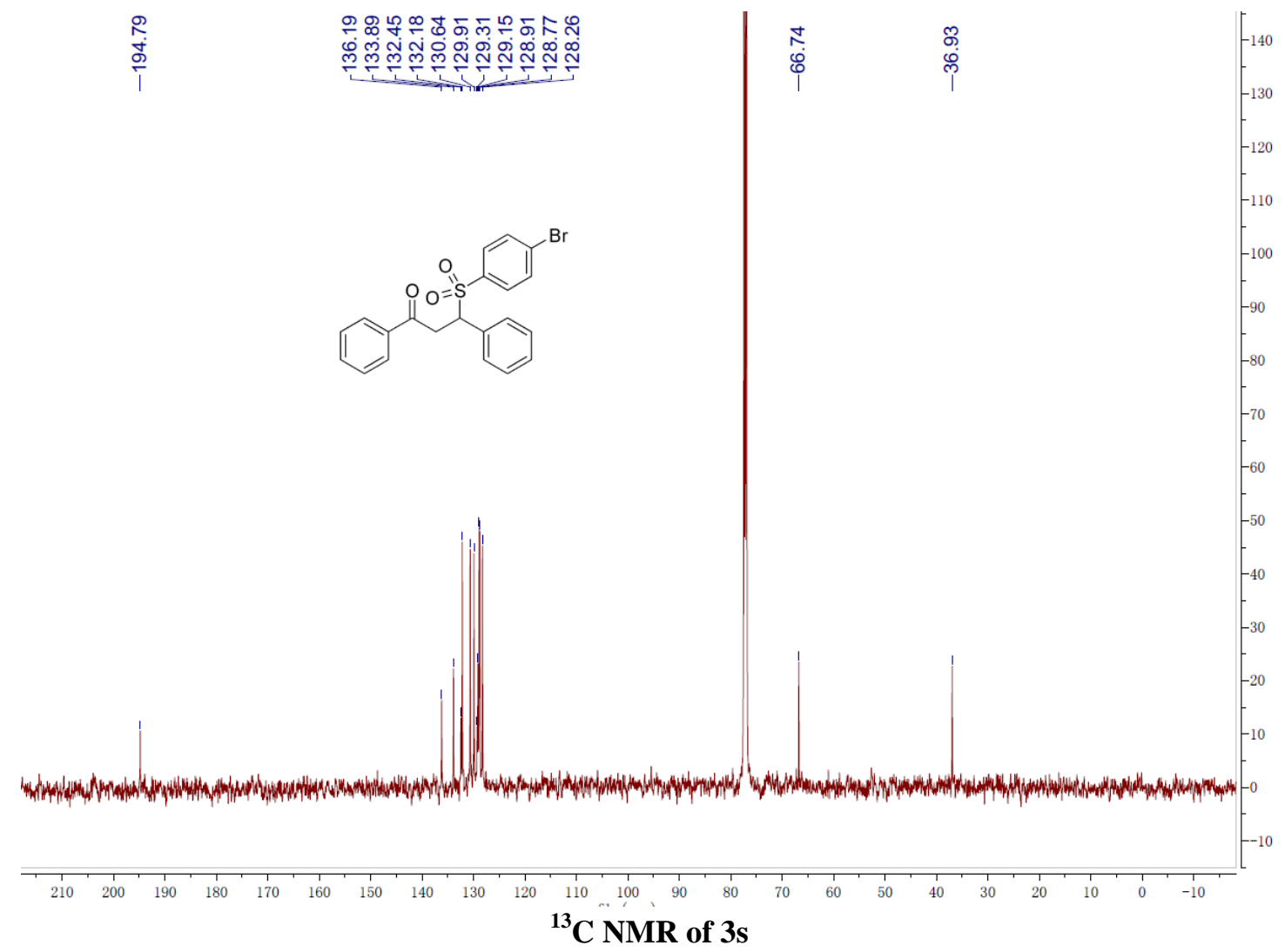



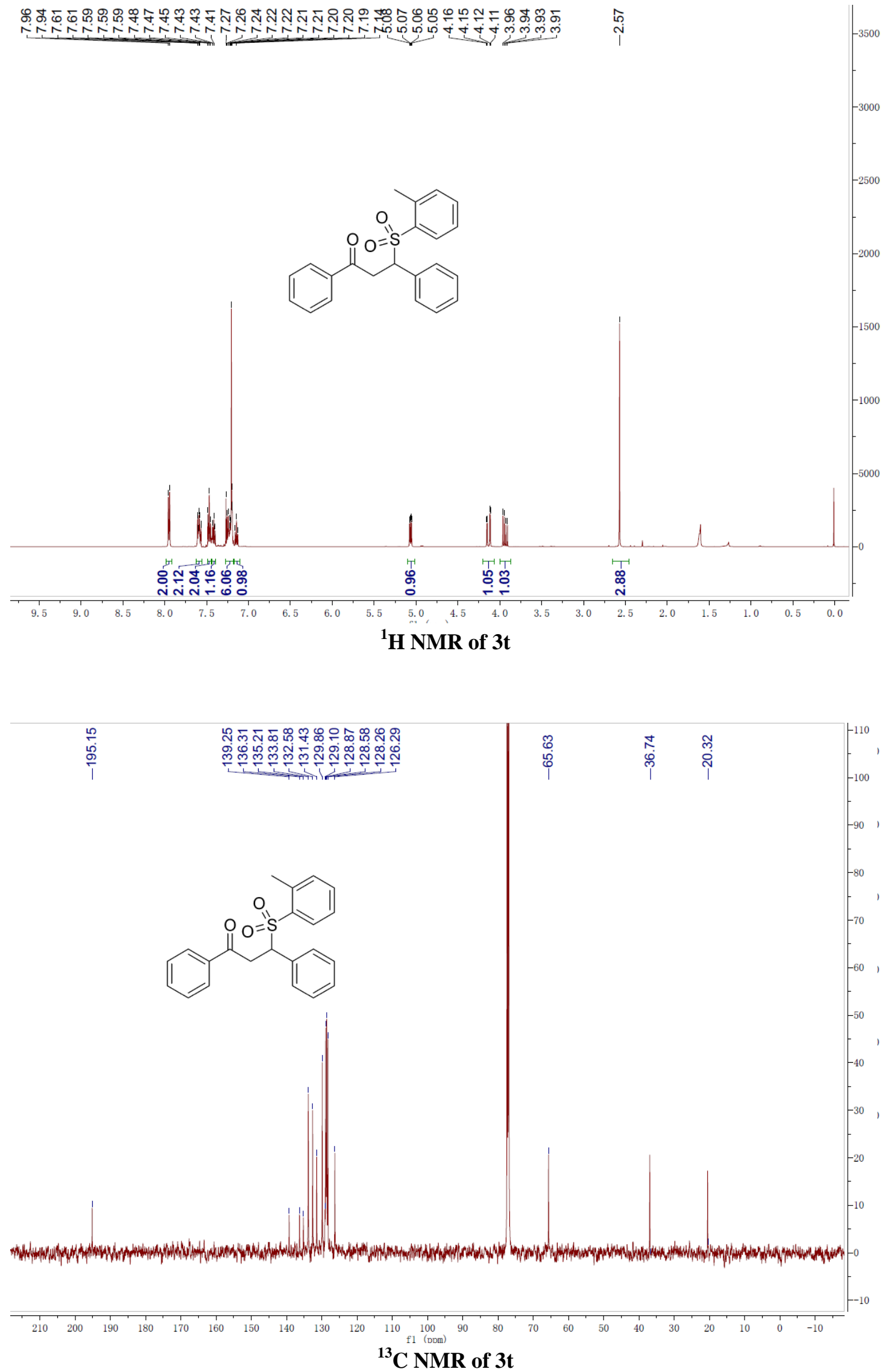

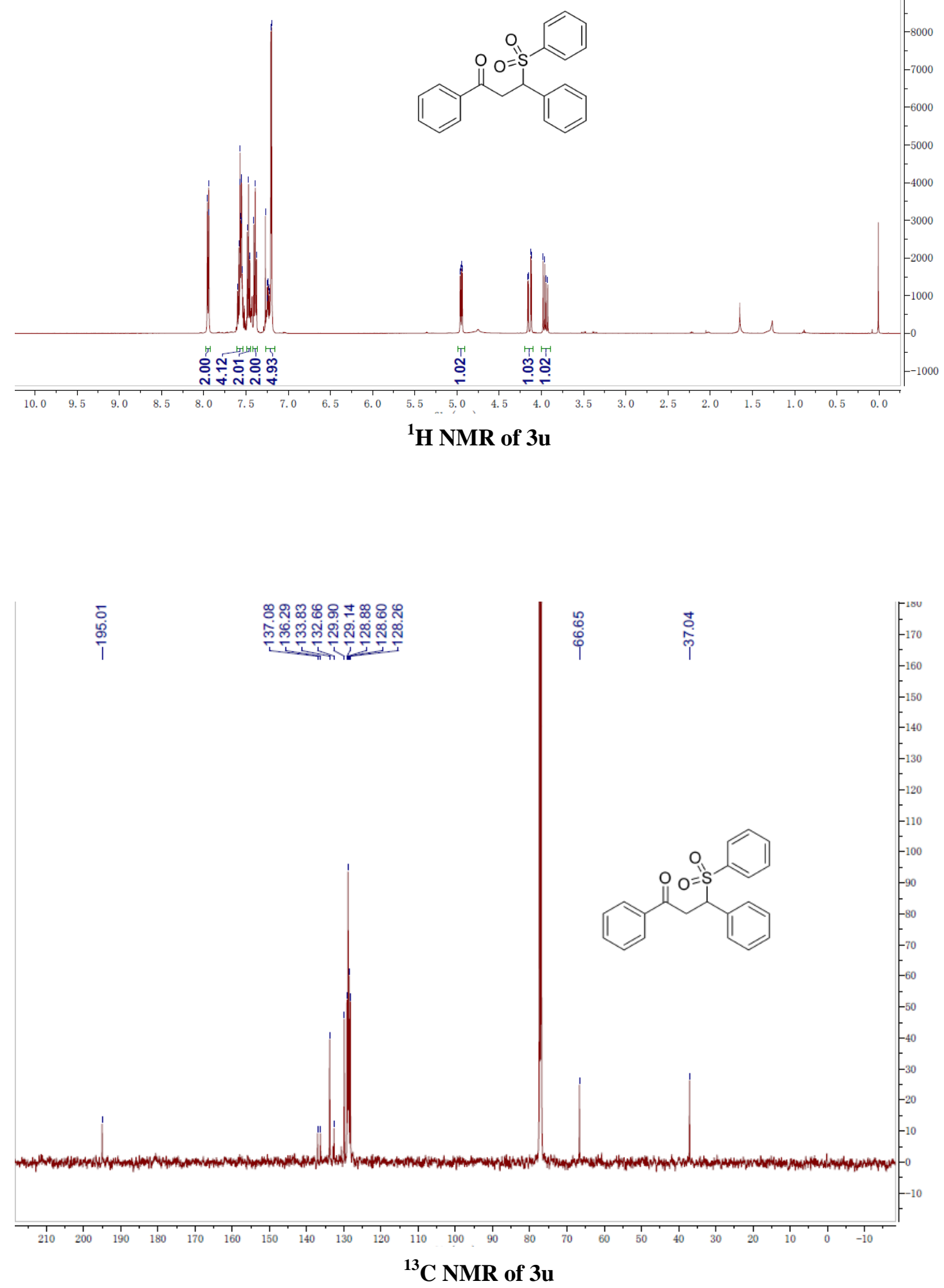


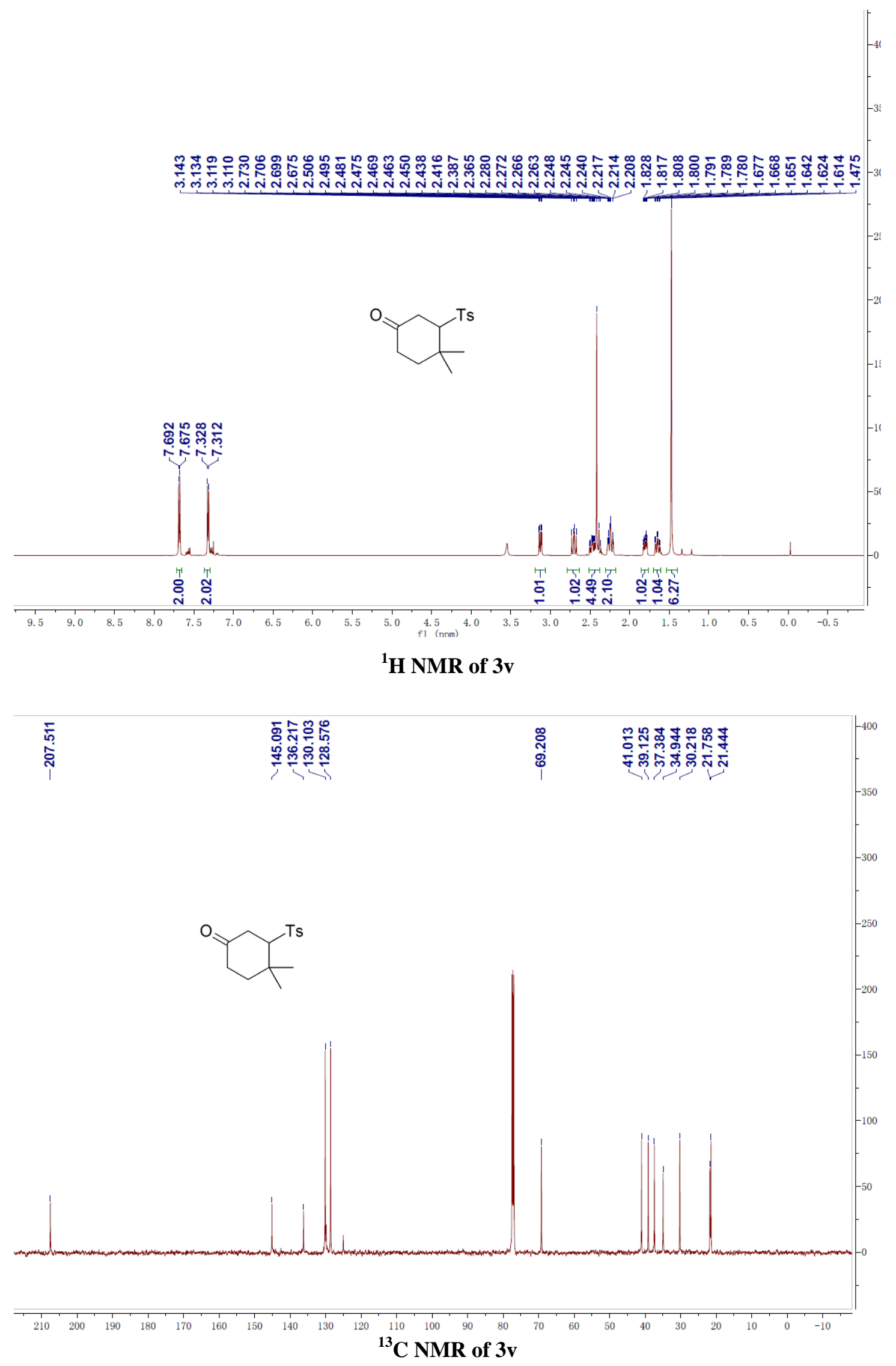



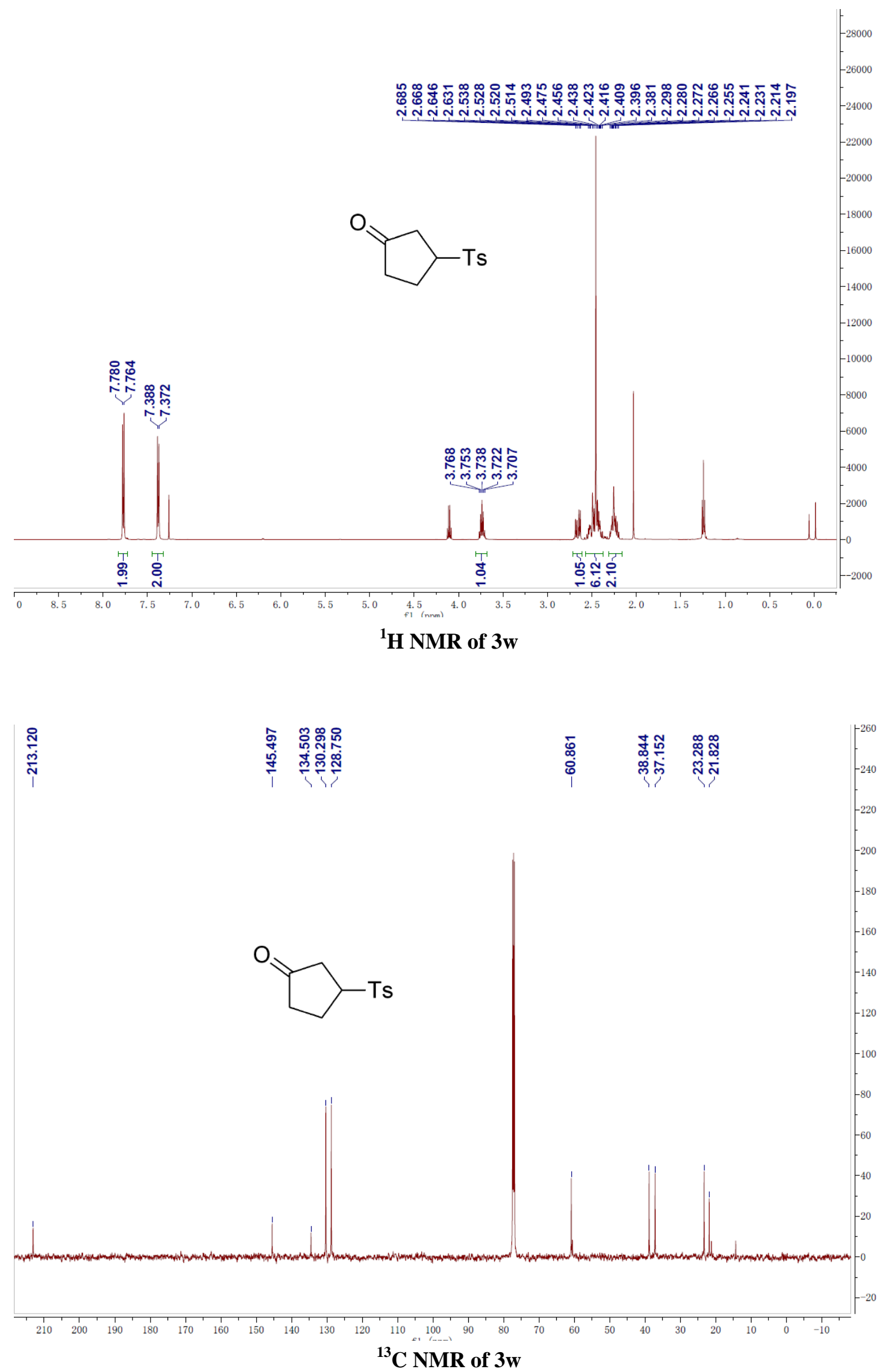


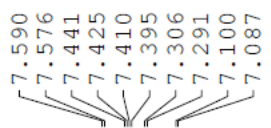

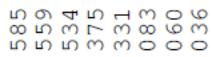

ن $\dot{v} \dot{v} \dot{\sim} \dot{v}$

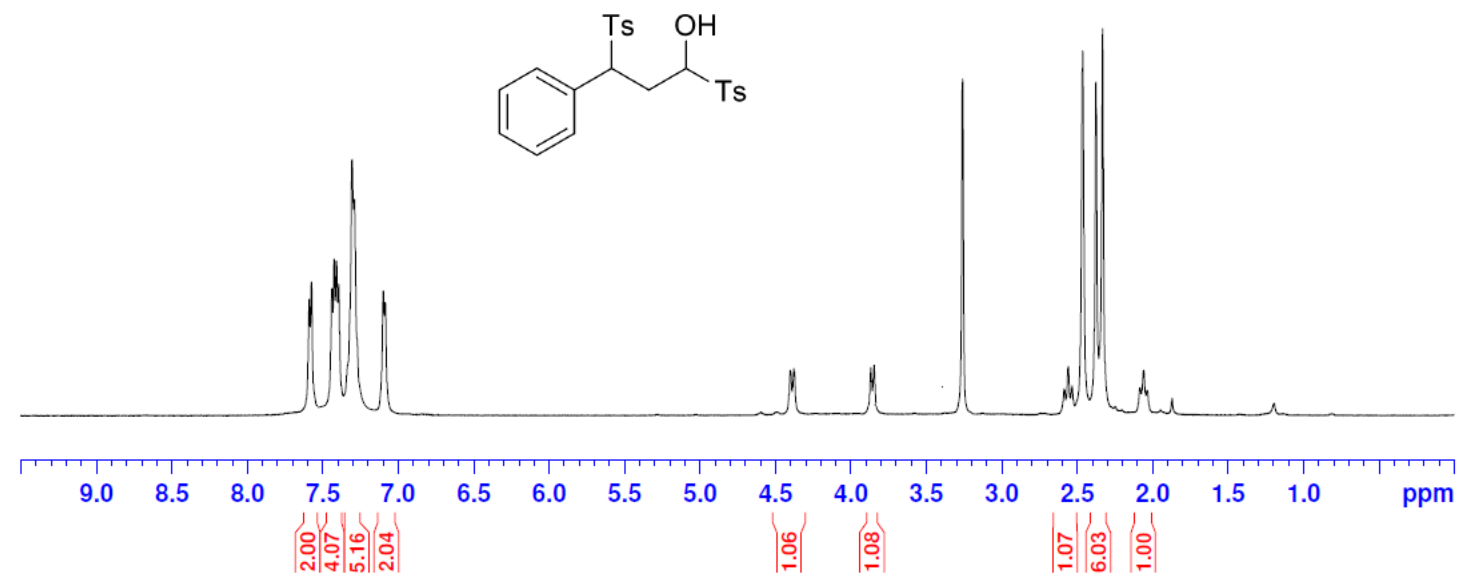

${ }^{1}$ H NMR of 4

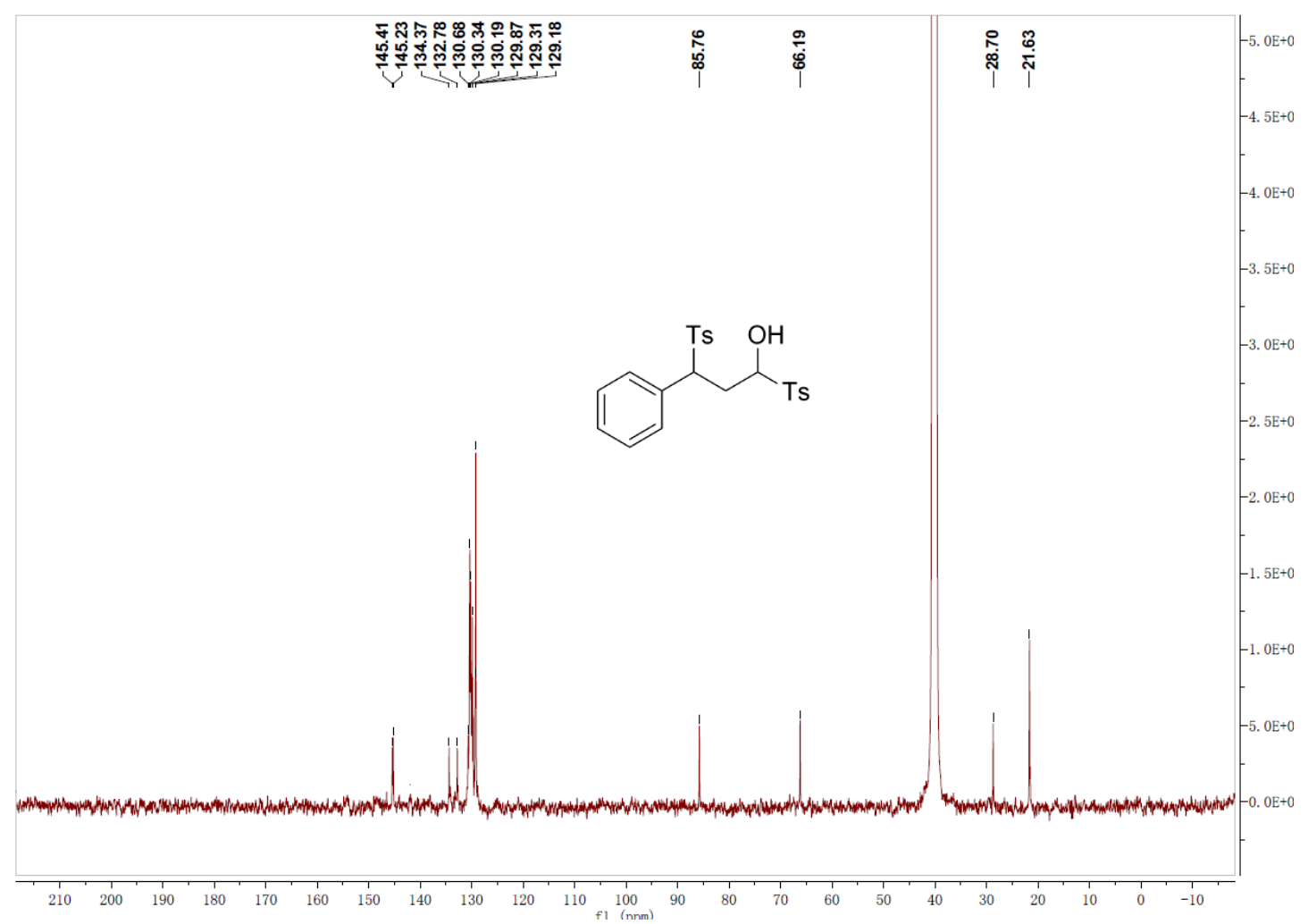

${ }^{1}$ H NMR of 4 


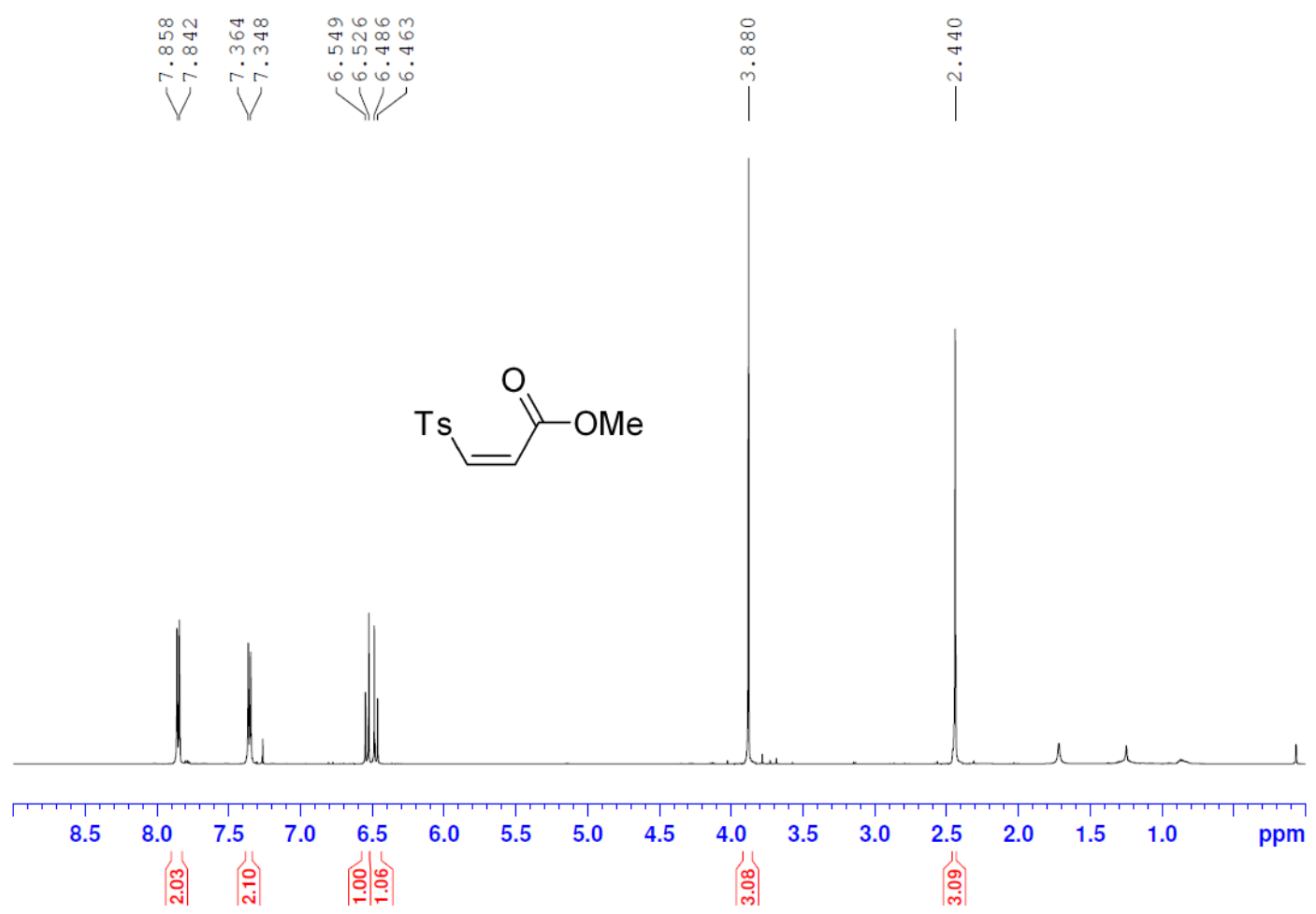

${ }^{1}$ H NMR of 5

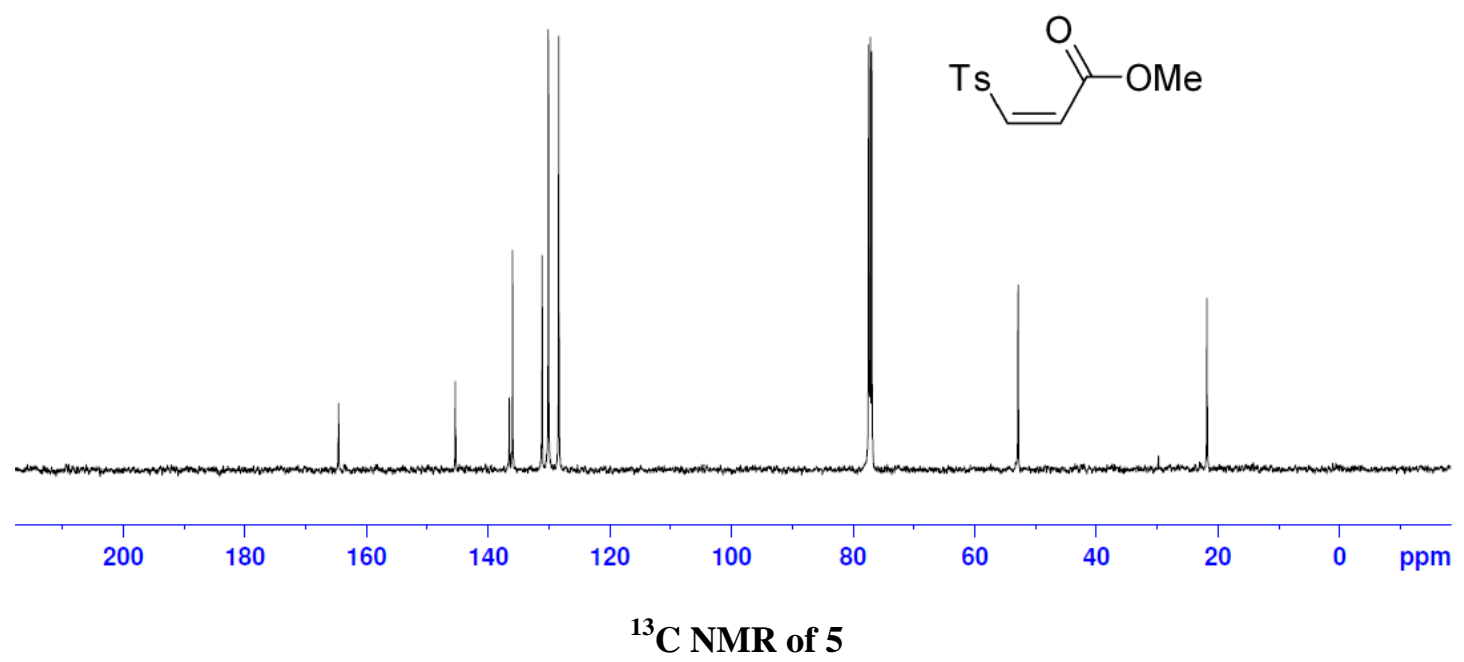



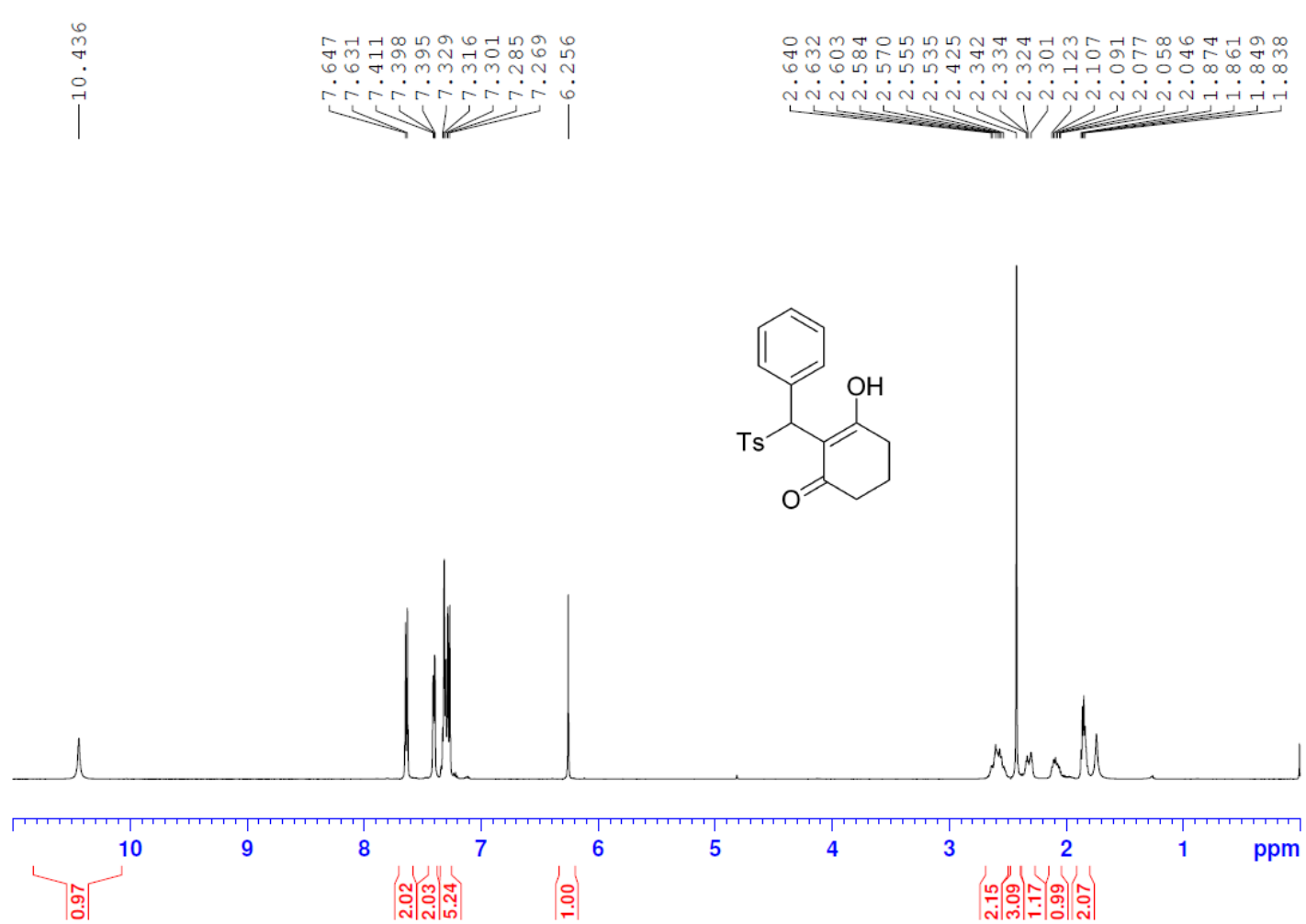

${ }^{1} \mathrm{H}$ NMR of $6 \mathrm{a}$

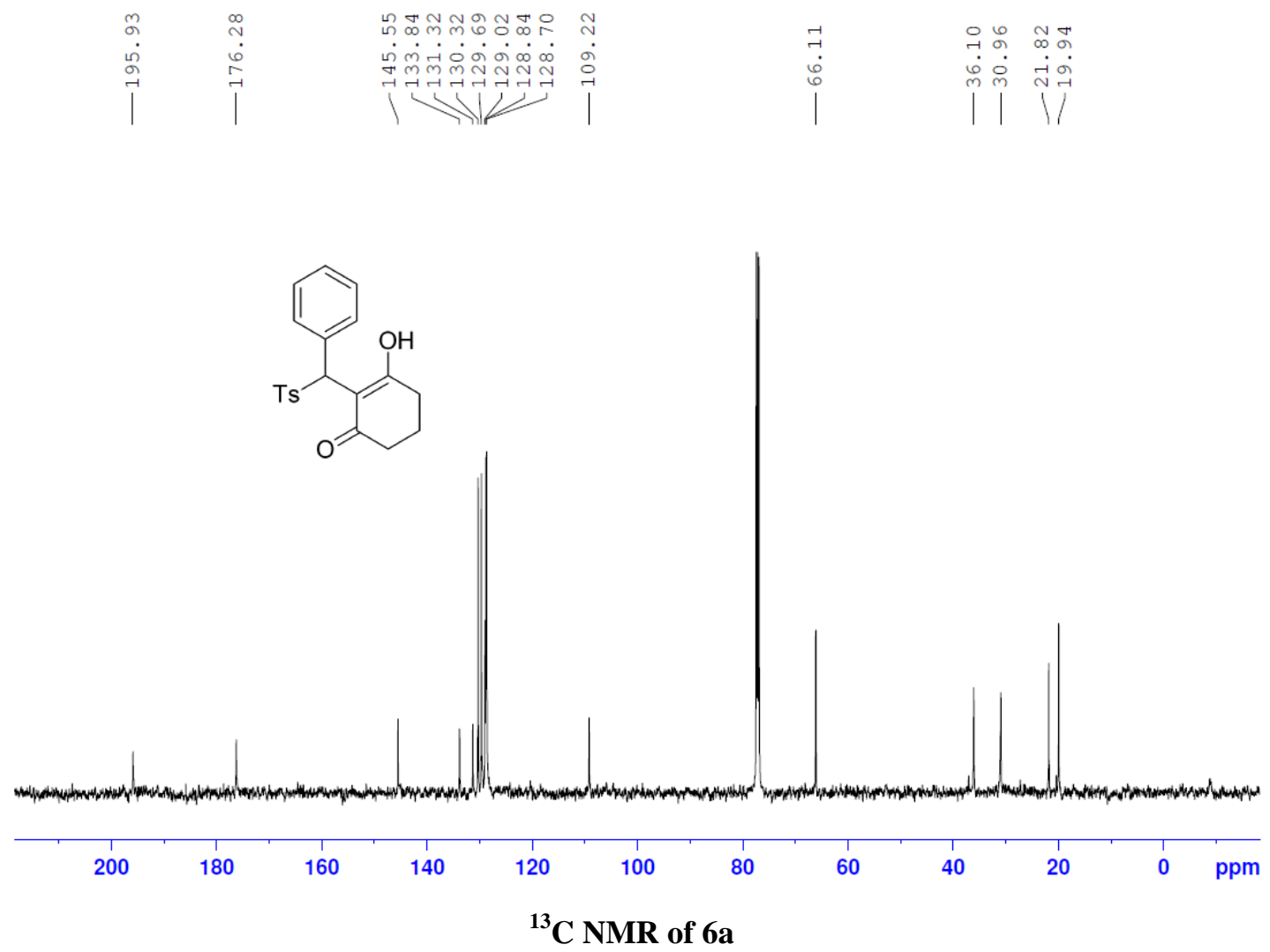




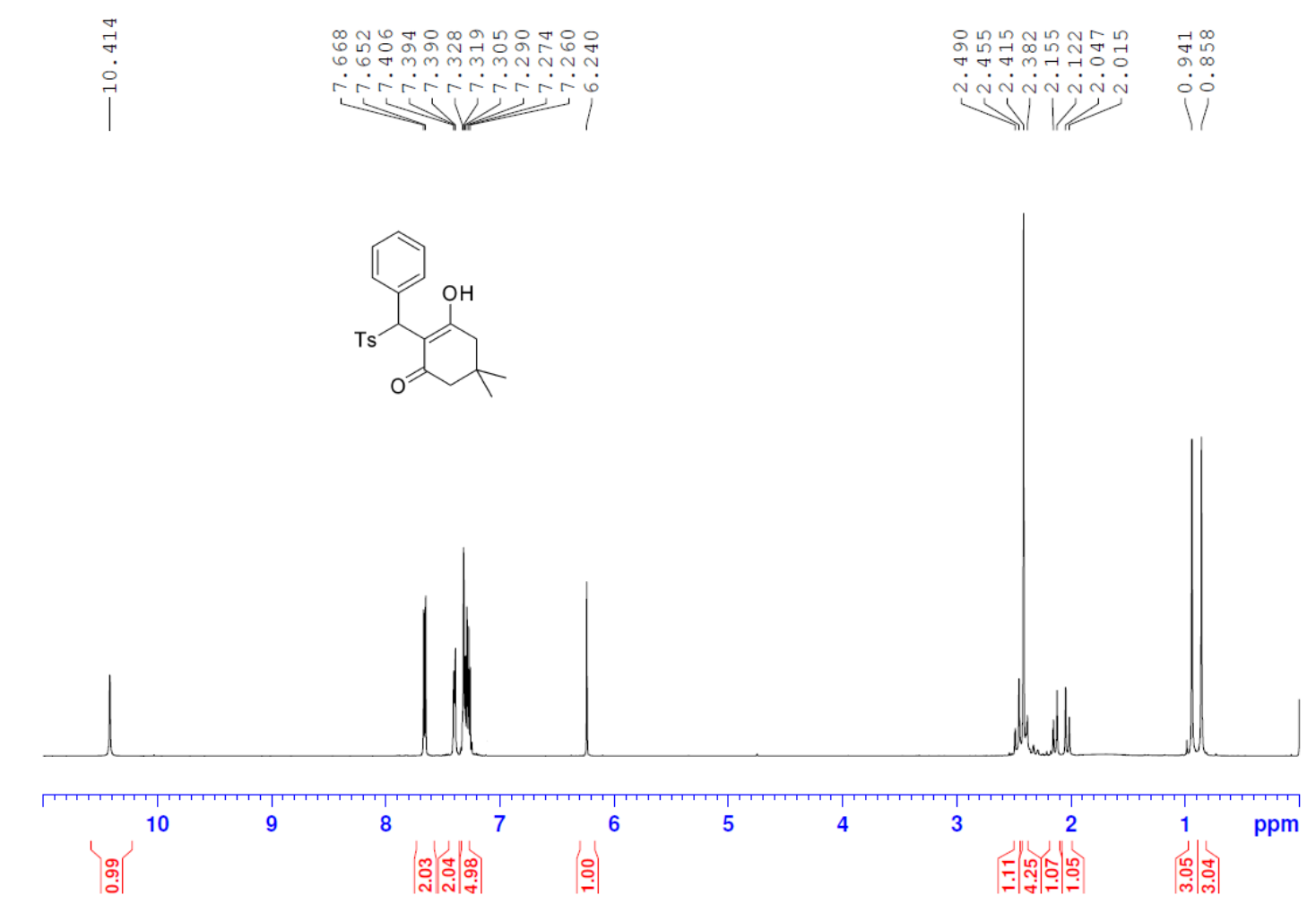

${ }^{1}$ H NMR of $6 b$

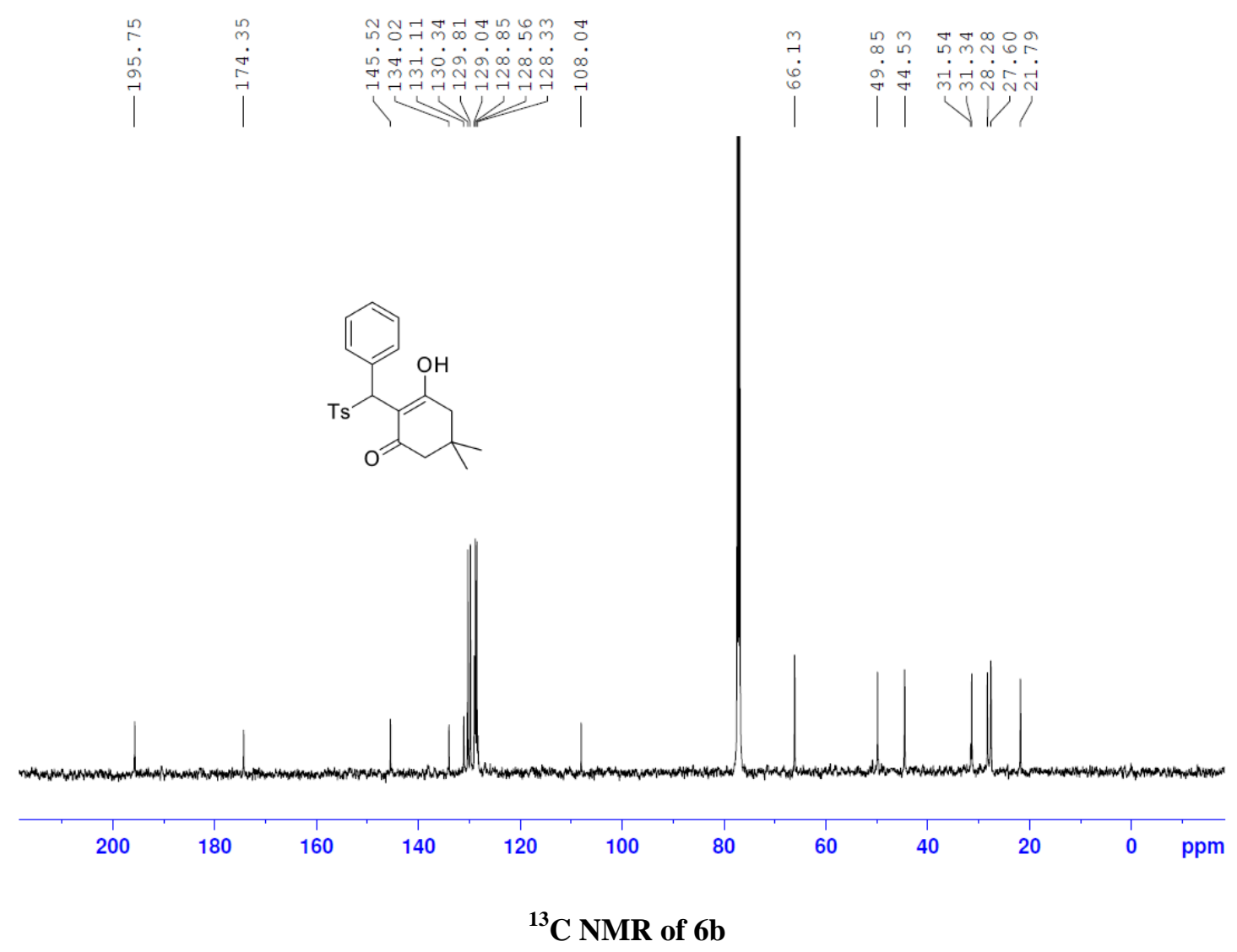



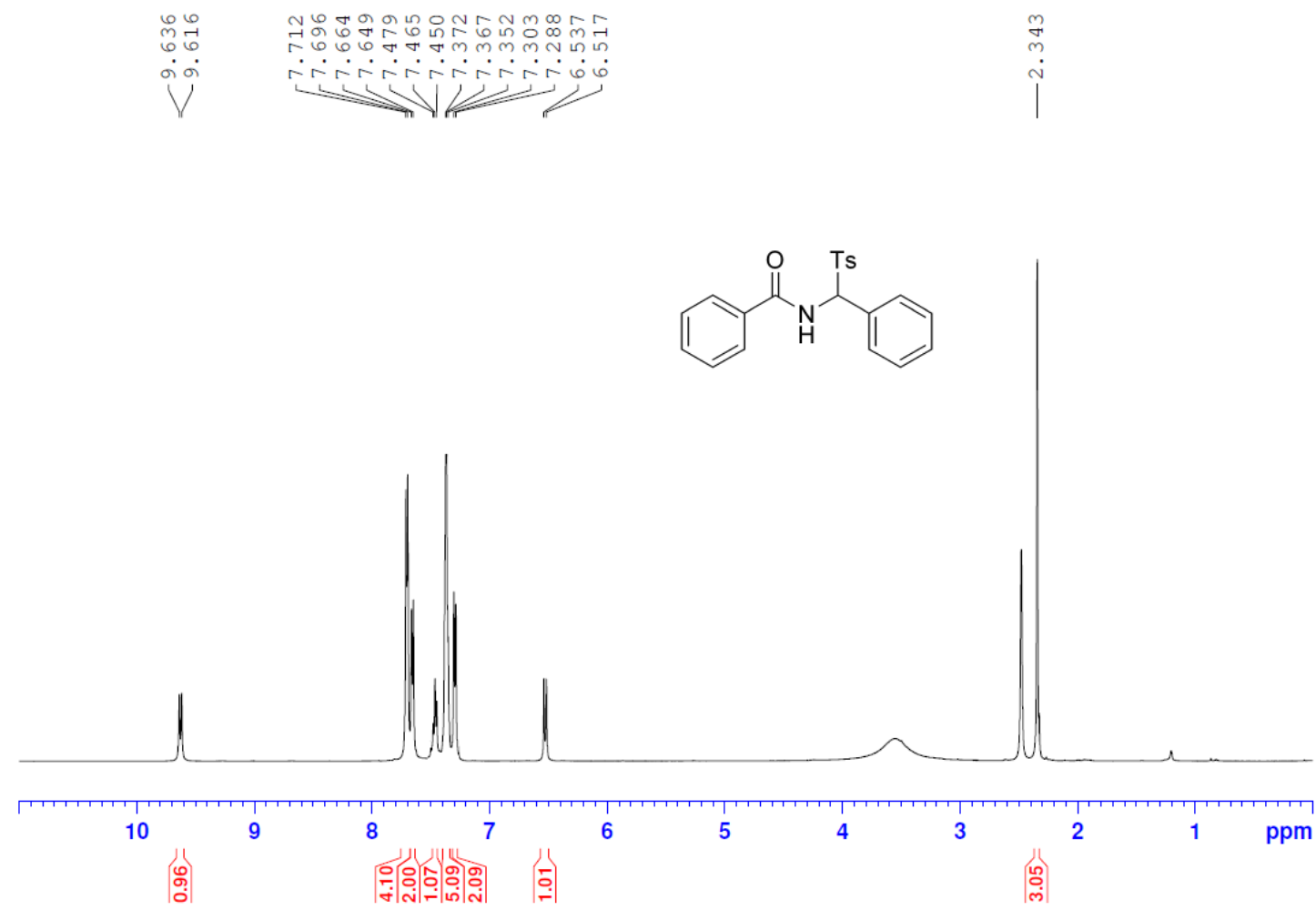

${ }^{1} \mathrm{H}$ NMR of $7 \mathrm{a}$

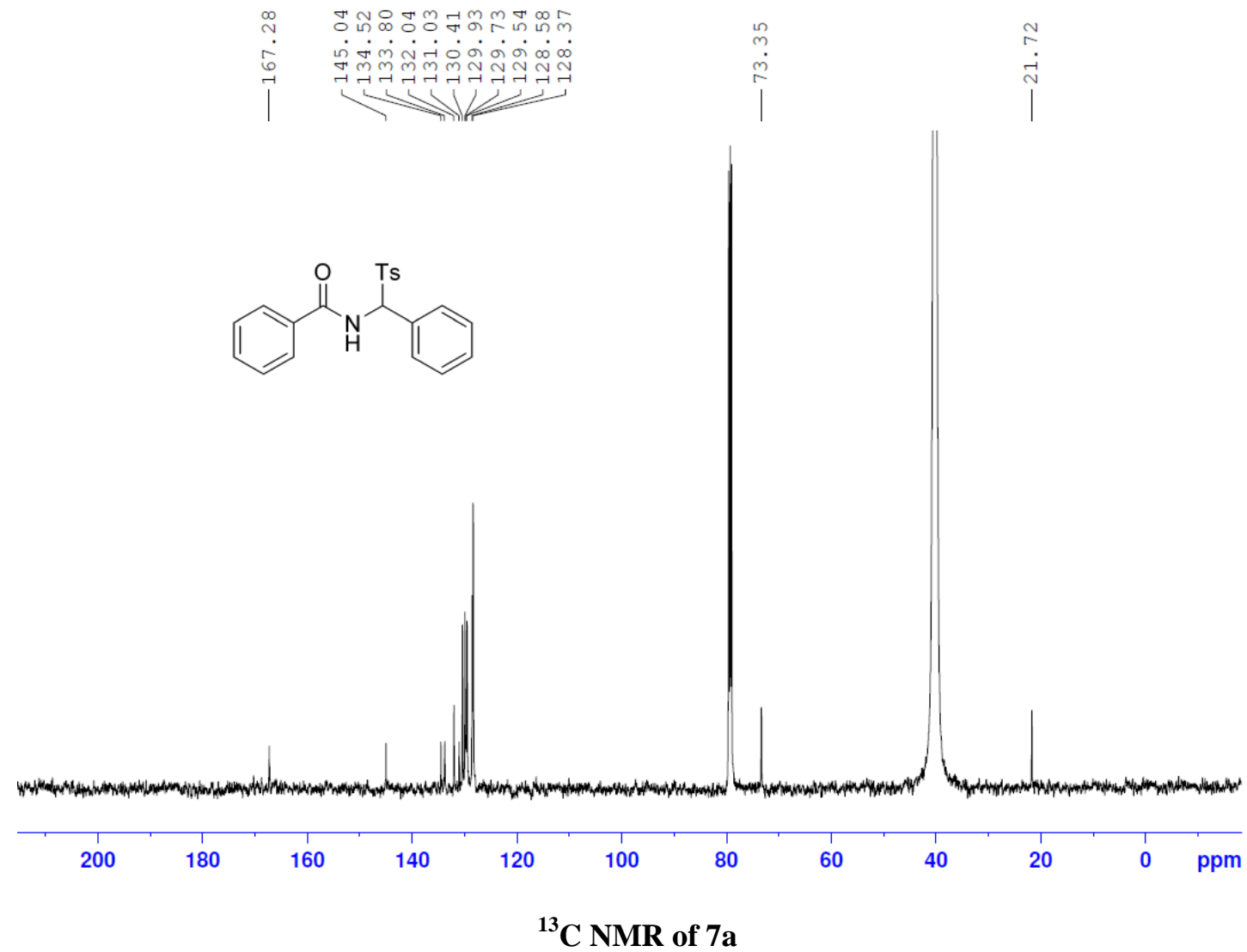




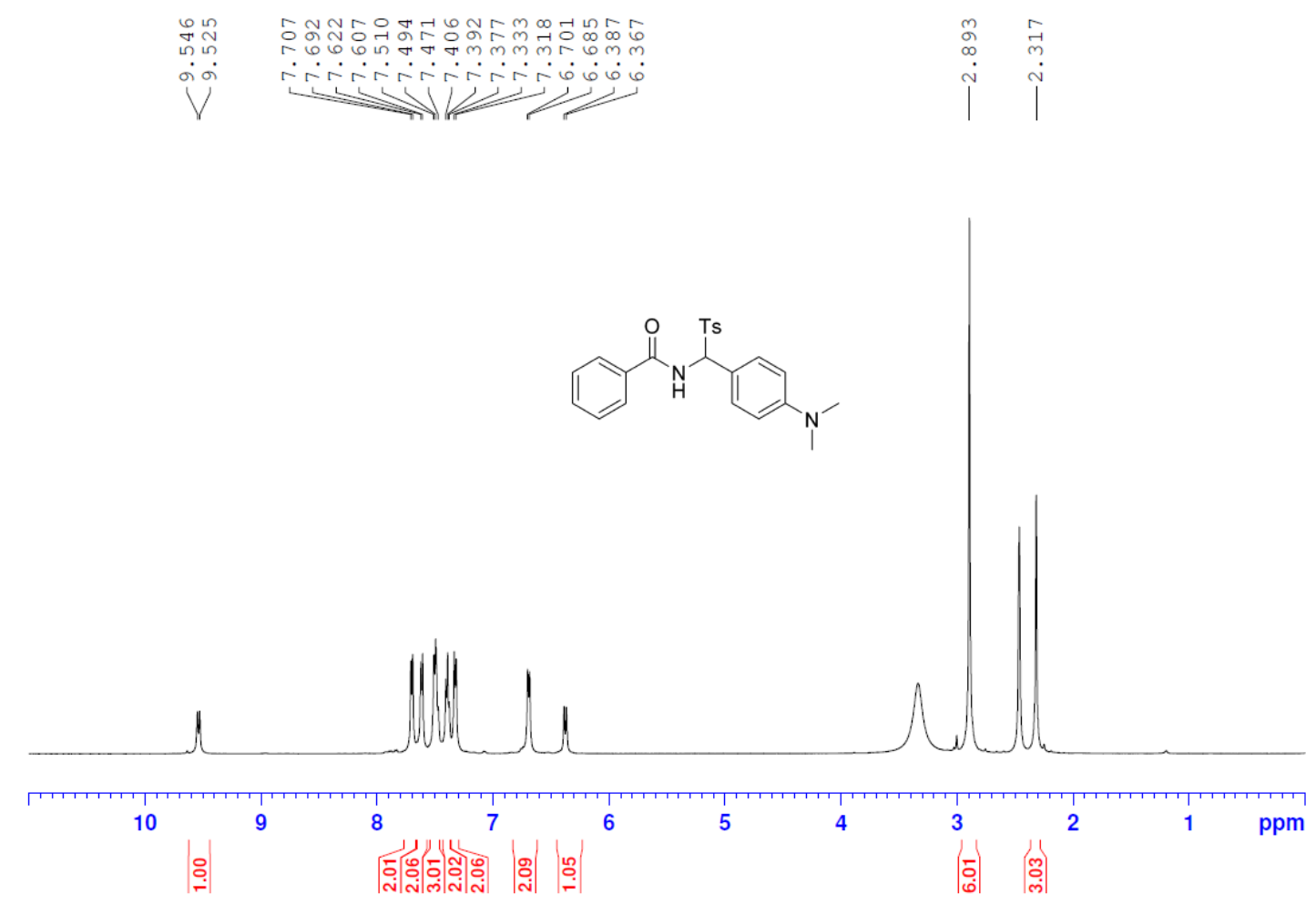

${ }^{1} \mathrm{H}$ NMR of $7 \mathrm{~b}$

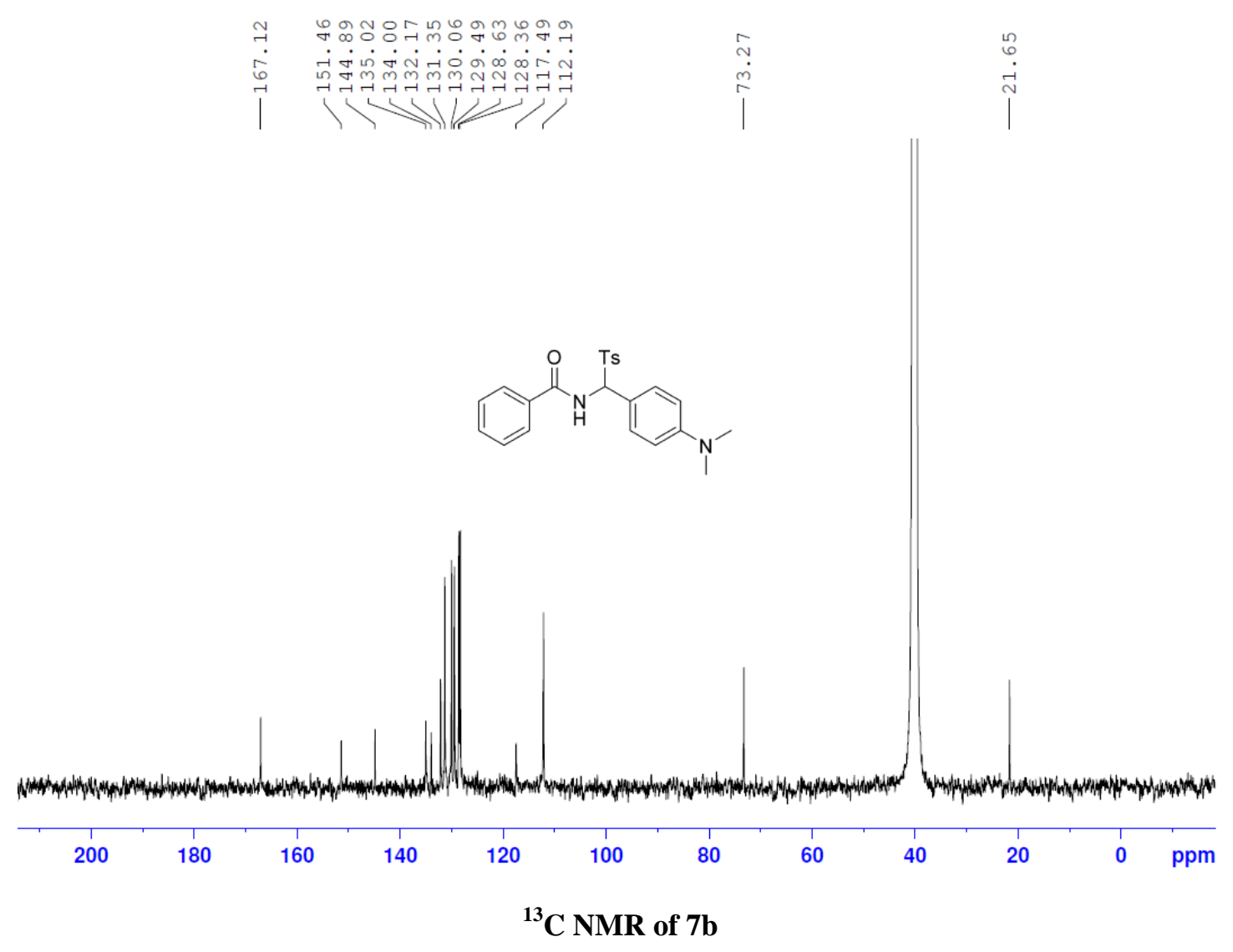

